\title{
A splitter theorem for 3-connected 2-polymatroids
}

\author{
James Oxley \\ Department of Mathematics \\ Louisiana State University \\ Baton Rouge, U.S.A. \\ oxley@math.1su.edu
}

\author{
Charles Semple \\ Department of Mathematics and Statistics \\ University of Canterbury \\ Christchurch, New Zealand \\ charles.semple@canterbury.ac.nz
}

\section{Geoff Whittle}

School of Mathematics, Statistics and Operations Research

Victoria University

Wellington, New Zealand

geoff.whittle@vuw.ac.nz

Submitted: Sep 12, 2017; Accepted: May 7, 2019; Published: May 31, 2019

(c) The authors. Released under the CC BY-ND license (International 4.0).

\begin{abstract}
Seymour's Splitter Theorem is a basic inductive tool for dealing with 3-connected matroids. This paper proves a generalization of that theorem for the class of 2polymatroids. Such structures include matroids, and they model both sets of points and lines in a projective space and sets of edges in a graph. A series compression in such a structure is an analogue of contracting an edge of a graph that is in a series pair. A 2-polymatroid $N$ is an s-minor of a 2-polymatroid $M$ if $N$ can be obtained from $M$ by a sequence of contractions, series compressions, and dual-contractions, where the last are modified deletions. The main result proves that if $M$ and $N$ are 3-connected 2-polymatroids such that $N$ is an s-minor of $M$, then $M$ has a 3-connected s-minor $M^{\prime}$ that has an s-minor isomorphic to $N$ and has $|E(M)|-1$ elements unless $M$ is a whirl or the cycle matroid of a wheel. In the exceptional case, such an $M^{\prime}$ can be found with $|E(M)|-2$ elements.
\end{abstract}

Mathematics Subject Classifications: 05B35

\section{Introduction}

Let $M$ be a 3-connected matroid other than a wheel or a whirl. Tutte [21] proved that $M$ has an element whose deletion or contraction is 3-connected. Seymour [19] extended this theorem by showing that, for a proper 3-connected minor $N$ of $M$, the matroid $M$ has an 
element whose deletion or contraction is 3-connected and has an $N$-minor. These theorems have been powerful inductive tools for working with 3-connected matroids. In [16], with a view to attacking representability problems for 2-polymatroids, we generalized the Wheelsand-Whirls Theorem to 2-polymatroids. In this paper, we prove a generalization of the Splitter Theorem for 2-polymatroids.

A basic example of a matroid is a set of points in a projective space. If, instead, we take a finite set of points and lines in a projective space, we get an example of a 2-polymatroid. Whereas each element of a matroid has rank zero or one, an individual element in a 2-polymatroid can also have rank two. Formally, for a positive integer $k$, a $k$-polymatroid $M$ is a pair $(E, r)$ consisting of a finite set $E$, called the ground set, and a function $r$, called the rank function, from the power set of $E$ into the integers satisfying the following conditions:

(i) $r(\emptyset)=0$;

(ii) if $X \subseteq Y \subseteq E$, then $r(X) \leqslant r(Y)$;

(iii) if $X$ and $Y$ are subsets of $E$, then $r(X)+r(Y) \geqslant r(X \cup Y)+r(X \cap Y)$; and

(iv) $r(\{e\}) \leqslant k$ for all $e \in E$.

A matroid is just a 1-polymatroid. Equivalently, it is a 2-polymatroid in which every element has rank at most one. Our focus in this paper will be on 2-polymatroids. From a graph $G$, in addition to its cycle matroid, we can derive a second 2-polymatroid on $E(G)$, which we denote by $M_{2}(G)$. The latter is defined by letting the rank of a set $A$ of edges be the number of vertices incident with edges in $A$. Observe that non-loop edges of $G$ have rank two in $M_{2}(G)$.

Matroid connectivity generalizes naturally to 2-polymatroids. In particular, 3-connectivity for matroids extends routinely to a notion of 3 -connectivity for 2 -polymatroids. A simple 3-connected graph $G$ has a 3-connected cycle matroid. On the other hand, $M_{2}(G)$ is 3-connected whenever $G$ is a 2-connected loopless graph.

Deletion and contraction for matroids extend easily to 2-polymatroids. This gives a notion of minor for 2-polymatroids that extends that of minor for matroids, and, via cycle matroids, that of minor for graphs. But what happens when we consider the 2polymatroid $M_{2}(G)$ ? If $e$ is an edge of $G$, then deletion in $M_{2}(G)$ corresponds to deletion in $G$, but it is not the same with contraction. However, there is an operation on $M_{2}(G)$ that corresponds to contraction in $G$. Specifically, if $e$ is an element of the 2-polymatroid $M$ and $r(\{e\})>0$, then the compression of $e$ from $M$, denoted $M \downarrow e$, is obtained by placing a rank-1 element $x$ freely on $e$, contracting $x$, and then deleting $e$ from the resulting 2-polymatroid. In particular, $M_{2}(G) \downarrow e=M_{2}(G / e)$ for a non-loop edge $e$ of the graph $G$.

Representability of matroids extends easily to representability of polymatroids over fields. Indeed, much of the motivation for this paper is derived from our desire to develop tools for attacking representability problems for 2-polymatroids. The class of 2polymatroids representable over a field $\mathbb{F}$ is closed under both deletion and contraction. 
When $\mathbb{F}$ is finite, this is not the case for compression in general although it is the case for a restricted type of compression. In [16], we defined a certain type of 3-separator, which we called a 'prickly' 3 -separator. A series pair in a graph $G$ is a 2-element prickly 3-separator of $M_{2}(G)$. Larger prickly 3-separators do not arise from graphs, but do arise in more general settings. Compressing elements from prickly 3-separators preserves representability. We gave examples in [16] to show that, if we wish to generalize Tutte's Wheels-and-Whirls Theorem to 2-polymatroids, it is necessary to allow compression of elements from prickly 3 -separators. The main result of [16] proves such a generalization by showing that a 3-connected non-empty 2-polymatroid that not a whirl or the cycle matroid of a wheel has an element $e$ such that either $M \backslash e$ or $M / e$ is 3-connected, or $e$ belongs to a prickly 3 -separator, and $M \downarrow e$ is 3-connected.

Geelen, Gerards, and Whittle [4] have announced that Rota's Conjecture [20] is true, that is, for every finite field, there is a finite set of minor-minimal matroids that are not representable over that field. In [16], we showed that, for every field $\mathbb{F}$, the set of minorminimal 2-polymatroids that are not representable over $\mathbb{F}$ is infinite, so one generalization of Rota's Conjecture for 2-polymatroids fails. We believe, however, that an alternative generalization of the conjecture does hold. Specifically, we conjectured in [16] that, when $\mathbb{F}$ is finite, there are only finitely many 2-polymatroids that are minimal with the property of being non-representable over $\mathbb{F}$ where we allow, as reduction operations, not only deletion and contaction but also compression of elements from prickly 3-separators.

Our main result appears at the end of this section. We now give the rest of the background needed to understand that result. The matroid terminology used here will follow Oxley [14]. Lovász and Plummer [10, Chapter 11] have given an interesting discussion of 2-polymatroids and some of their properties. We call $(E, r)$ a polymatroid if it is a $k$-polymatroid for some positive integer $k$. In a 2-polymatroid $(E, r)$, an element $x$ will be called a line, a point, or a loop when its rank is 2, 1, or 0, respectively. For readers accustomed to using the terms 'point' and 'line' for flats in a matroid of rank one and two, respectively, this may create some potential confusion. However, in this paper, we shall never use the terms 'point' and 'line' in this alternative way. Indeed, we will not even define a flat of a 2-polymatroid.

Let $M$ be a polymatroid $(E, r)$. For a subset $X$ of $E$, the deletion $M \backslash X$ and the contraction $M / X$ of $X$ from $M$ are the pairs $\left(E-X, r_{1}\right)$ and $\left(E-X, r_{2}\right)$ where, for all subsets $Y$ of $E-X$, we have $r_{1}(Y)=r(Y)$ and $r_{2}(Y)=r(Y \cup X)-r(X)$. We shall also write $M \mid(E-X)$ for $M \backslash X$. A minor of the polymatroid $M$ is any polymatroid that can be obtained from $M$ by a sequence of operations each of which is a deletion or a contraction. It is straightforward to check that every minor of a $k$-polymatroid is also a $k$-polymatroid. The closure $\operatorname{cl}(X)$ of a set $X$ in $M$ is, as for matroids, the set $\{x \in E: r(X \cup x)=r(X)\}$. Two polymatroids $\left(E_{1}, r_{1}\right)$ and $\left(E_{2}, r_{2}\right)$ are isomorphic if there is a bijection $\phi$ from $E_{1}$ onto $E_{2}$ such that $r_{1}(X)=r_{2}(\phi(X))$ for all subsets $X$ of $E_{1}$.

One natural way to obtain a polymatroid is from a collection of flats of a matroid $M$. Indeed, every polymatroid arises in this way $[6,9,12]$. More precisely, we have the following.

Theorem 1. Let $t$ be a function defined on the power set of a finite set E. Then $(E, t)$ 
is a polymatroid if and only if, for some matroid $M$, there is a function $\psi$ from $E$ into the set of flats of $M$ such that $t(X)=r_{M}\left(\cup_{x \in X} \psi(x)\right)$ for all subsets $X$ of $E$.

The key idea in proving this theorem is that of freely adding a point to an element of a polymatroid. Let $(E, r)$ be a polymatroid, let $x$ be an element of $E$, and let $x^{\prime}$ be an element that is not in $E$. We can extend the domain of $r$ to include all subsets of $E \cup x^{\prime}$ by letting

$$
r\left(X \cup x^{\prime}\right)= \begin{cases}r(X), & \text { if } r(X \cup x)=r(X) ; \\ r(X)+1, & \text { if } r(X \cup x)>r(X) .\end{cases}
$$

Then it is not difficult to check that $\left(E \cup x^{\prime}, r\right)$ is a polymatroid. We say that it has been obtained from $(E, r)$ by freely adding $x^{\prime}$ to $x$. If we repeat this construction by freely adding a new element $y^{\prime}$ to some element $y$ of $E$, we can show that the order in which these two operations is performed is irrelevant.

Using this idea, we can associate a matroid with every 2-polymatroid $M$ as follows. Let $L$ be the set of lines of $M$. For each $\ell$ in $L$, freely add two points $s_{\ell}$ and $t_{\ell}$ to $\ell$. Let $M^{+}$be the 2-polymatroid obtained after performing all of these $2|L|$ operations. Let $M^{\prime}$ be $M^{+} \backslash L$. We call $M^{\prime}$ the natural matroid derived from $M$.

Given a graph $G$ with edge set $E$, as noted earlier, one can define a 2-polymatroid $M_{2}(G)$ on $E$ by, for each subset $X$ of $E$, letting $r(X)$ be $|V(X)|$ where $V(X)$ is the set of vertices of $G$ that have at least one endpoint in $X$. A polymatroid $\left(E^{\prime}, r^{\prime}\right)$ is Boolean if is isomorphic to the 2-polymatroid that is obtained in this way from some graph. One attractive feature of $M_{2}(G)$ is that, except for the possible presence of isolated vertices, it uniquely determines $G$. More precisely, if $G_{1}$ and $G_{2}$ are graphs neither of which has any isolated vertices and if $M_{2}\left(G_{1}\right)=M_{2}\left(G_{2}\right)$, then there is a labelling of the vertices of $G_{2}$ such that $G_{1}=G_{2}$. This contrasts with the situation for matroids where quite different graphs can have the same cycle matroids.

Let $M$ be a polymatroid $(E, r)$. The connectivity function, $\lambda_{M}$ or $\lambda$, of $M$ is defined, for all subsets $X$ of $E$, by $\lambda_{M}(X)=r(X)+r(E-X)-r(M)$. Observe that $\lambda_{M}(E-X)=$ $\lambda_{M}(X)$. It is routine to check, using the submodularity of the rank function, that the connectivity function is submodular, that is, for all subsets $Y$ and $Z$ of $E$,

$$
\lambda_{M}(Y)+\lambda_{M}(Z) \geqslant \lambda_{M}(Y \cup Z)+\lambda_{M}(Y \cap Z) .
$$

Let $M$ be a polymatroid. For a positive integer $n$, a subset $X$ of $E(M)$ is $n$-separating if $\lambda_{M}(X) \leqslant n-1$ and is exactly $n$-separating if $\lambda_{M}(X)=n-1$ We say that $M$ is 2connected if it has no proper non-empty 1-separating subset. We will also say that $M$ is disconnected if it is not 2-connected. We call $M$ 3-connected if $M$ is 2-connected and $M$ has no 2-separation, that is, $M$ has no partition $(X, Y)$ with $\max \{|X|, r(X)\}>1$ and $\max \{|Y|, r(Y)\}>1$ but $\lambda(X) \leqslant 1$. When $M$ is a 3 -connected 2-polymatroid $(E, r)$, a 3 -separation of $M$ is a partition $(X, Y)$ of $E$ such that $\lambda(X)=2$ and both $r(X)$ and $r(Y)$ exceed 2.

Duality plays a fundamental role in matroid theory and will also be important in our work with 2-polymatroids. Whereas there is a standard notion of what constitutes the dual 
of a matroid, for 2-polymatroids, there is more than one choice. Let $M$ be a $k$-polymatroid $(E, r)$. The $k$-dual of $M$ is the pair $\left(E, r_{k}^{*}\right)$ defined by $r_{k}^{*}(Y)=k|Y|+r(E-Y)-r(M)$. This notion of duality was used, for example, in Oxley and Whittle's treatment [17] of Tutte invariants for 2-polymatroids. An involution on the class $\mathcal{M}_{k}$ of $k$-polymatroids is a function $\zeta$ from $\mathcal{M}_{k}$ into $\mathcal{M}_{k}$ such that $\zeta(\zeta(M))=M$ for all $M$ in $\mathcal{M}_{k}$. Whittle [22] showed that the $k$-dual is the only involution on $\mathcal{M}_{k}$ under which deletion and contraction are interchanged in the familiar way. However, a disadvantage of this duality operation is that, for a matroid $M$, we can view $M$ as a $k$-polymatroid for all $k \geqslant 1$. Hence $M$ has a 1-dual, which is its usual matroid dual. But it also has a 2-dual, a 3-dual, and so on. In [16], we used a duality operation on the class of all polymatroids that, when applied to a $k$-polymatroid, produces another $k$-polymatroid and that, when applied to a matroid produces its usual matroid dual. In this paper, we will use a variant on that operation that agrees with it when applied to 3-connected 2-polymatroids with at least two elements.

Both of these versions of duality are members of a family of potential duals for a polymatroid $(E, r)$ that were defined by McDiarmid [12] and were based on assigning a weight $w(e)$ to each element $e$ of $E$ where $w(e) \geqslant r(\{e\})$ for all $e$ in $E$. For a set $X$, we shall write $\|X\|$ for the sum $\sum_{e \in X} w(e)$. In [16], we took $w(e)$ to be $\max \{r(\{e\}), 1\}$. Here, instead, we will take $w(e)=r(\{e\})$ and define the dual of a polymatroid $(E, r)$ to be the pair $\left(E, r^{*}\right)$ where, for all subsets $Y$ of $E$,

$$
r^{*}(Y)=\|Y\|+r(E-Y)-r(E)=\sum_{e \in Y} r(\{e\})+r(E-Y)-r(E) .
$$

It is straightforward to check that, when $(E, r)$ is a $k$-polymatroid, so too is $\left(E, r^{*}\right)$. When $M=(E, r)$, we shall write $M^{*}$ for $\left(E, r^{*}\right)$. When the polymatroid $M$ is a matroid, its dual as just defined coincides with its usual matroid dual provided $M$ has no loops. However, if $e$ is a loop of $M$, then $e$ is a loop of $M^{*}$. The definition of dual used in [16] (where we took $\|Y\|=\sum_{e \in Y} \max \{1, r(\{e\})\}$ ) was chosen to ensure that, when $M$ is a matroid, its polymatroid dual coincides with its matroid dual. Here, however, we are giving up on that, albeit in a rather specialized case. Note, however, that the two definitions of dual coincide unless $M$ has a loop so, in particular, they coincide when $M$ is 3 -connected having at least two elements. Moreover, as noted in [16], these two versions of duality share a number of important properties, the proofs of which are very similar. For example, $\lambda_{M}(X)=\lambda_{M^{*}}(X)$. Next we discuss the reason for the use of the above definition of duality, which follows $[7,8]$.

Consider the following example, which will guide how we proceed. Begin with the matroid that is the direct sum of $P G(r-1, q)$ and $P G(k-2, q)$ viewing this as a restriction of $P G(r+k-2, q)$. Let $N$ be the restriction of $P G(r-1, q)$ to the complement of a hyperplane $H$ of it, so $N \cong A G(r-1, q)$. Take $k$ distinct points, $x_{1}, x_{2}, \ldots, x_{k}$, of $P G(r-1, q)$ that are in $H$. Then $k \leqslant \frac{q^{r-1}-1}{q-1}$. Let $\left\{y_{1}, y_{2}, \ldots, y_{k}\right\}$ be a spanning circuit in $P G(k-2, q)$. For each $i$ in $\{1,2, \ldots, k\}$, let $\ell_{i}$ be the rank-2 flat of $P G(r+k-2, q)$ that is spanned by $\left\{x_{i}, y_{i}\right\}$. Let $M$ be the 2-polymatroid whose elements are the points of $N$ along with the set $L$ consisting of the lines $\ell_{1}, \ell_{2}, \ldots, \ell_{k}$. It is straightforward to check 
that $M$ and $N$ are 3 -connected. The only way to obtain an $N$-minor of $M$ is to delete all the elements of $L$ since contracting any member of $L$ has the effect of reducing the rank of $E(N)$. But, in each of the 2-polymatroids $M \backslash L^{\prime}$, where $L^{\prime}$ is a proper non-empty subset of $L-\ell_{k}$, the set $\ell_{k}$ is 2-separating. Since our goal is a splitter theorem, where we can remove some bounded number of elements from $M$ maintaining both 3-connectivity and an $N$-minor, we will need a strategy for dealing with this example. One significant feature of this example is the very constrained nature of the 2-separations in each $M \backslash L^{\prime}$ with one side of each such 2-separation consisting of a single line. This is reminiscent of what happens in Bixby's Lemma [2] for 3-connected matroids where, for every element $e$ of such a matroid $N$, either $N \backslash e$ is 3-connected except for some possible series pairs, or $N / e$ is 3-connected except for some possible parallel pairs. Indeed, in the matroid derived from $M \backslash L^{\prime}$, each 2-separating line of $M \backslash L^{\prime}$ yields a series pair in the derived matroid.

The strategy that we will adopt is intimately linked to our choice of definition for the dual of a polymatroid. It is well known that, under the familiar definition of duality for matroids, taking the dual of the dual returns us to the original matroid. We now consider the relationship between a polymatroid $M$ and the polymatroid $\left(M^{*}\right)^{*}$. If $M$ is a 3-connected 2-polymatroid with at least two elements, then $\left(M^{*}\right)^{*}=M$. To see what happens in general, we follow [8]. Let $M$ be the polymatroid $(E, r)$. An element $e$ of $M$ is compact if $r(\{e\})=\lambda_{M}(\{e\})$ or, equivalently, if $r(E-\{e\})=r(E)$. We call $M$ compact if every element is compact. Thus, for example, a matroid is compact if it has no coloops. In the example in the last paragraph, although $M$ is compact, $M \backslash\left\{\ell_{1}\right\}$ is not since, for each $i \geqslant 2$, we have $r\left(\left\{\ell_{i}\right\}\right)=2$ whereas $\lambda_{M \backslash\left\{\ell_{1}\right\}}\left(\left\{\ell_{i}\right\}\right)=1$.

The compactification $M^{b}$ of the polymatroid $M$ is the pair $\left(E, r^{b}\right)$ where

$$
r^{b}(X)=r(X)+\sum_{x \in X}[\lambda(\{x\})-r(\{x\})]
$$

for all subsets $X$ of $E$. It is shown in [8] that $M^{b}$ is a compact polymatroid and it is clear that if $M$ is a 2-polymatroid, then so is $M^{b}$. The next result [8] encapsulates some key properties of this compactification operation and justifies the approach we take here.

Lemma 2. Let $(E, r)$ be a polymatroid $M$ and suppose $X \subseteq E$. Then

(i) $M^{*}$ is compact;

(ii) $\left(M^{*}\right)^{*}=M^{b}$;

(iii) $\lambda_{M}=\lambda_{M^{*}}=\lambda_{M^{b}}$;

(iv) $(M / X)^{*}=\left(M^{*} \backslash X\right)^{b}$; and

(v) if $M$ is compact, then $M / X$ is compact.

Returning to our guiding example above, although $M \backslash\left\{\ell_{1}\right\}$ is neither compact nor 3 -connected, its compactification is both. Observe that this compactification can be obtained from the restriction of the matroid $P G(r-1, q)$ to $E(N) \cup\left\{x_{2}, x_{3}, \ldots, x_{k}\right\}$ by 
relabelling each $x_{i}$ by $\ell_{i}$ noting that these $\ell_{i}$ are now points rather than lines. Thus compactification here has an analogous effect to cosimplification in matroids. By incorporating compactification as part of the deletion operation, which is justified by (iv) of the last lemma, we see that, after deleting a single element, we have both maintained 3 -connectivity and kept an $N$-minor. This is precisely what we want in a splitter theorem.

In 2-polymatroids, the behaviour of contraction differs significantly from that for matroids. In particular, consider the 2-polymatroid $M_{2}(G)$ obtained from a graph $G$, where $G$ has vertex set $V$ and edge set $E$. Let $e$ be an edge of $G$. Deleting $e$ from $G$ has an unsurprising effect; specifically, $M_{2}(G) \backslash e=M_{2}(G \backslash e)$. But, to find $M_{2}(G) / e$, we cannot simply look at $M_{2}(G / e)$. In particular, what do we do with elements whose rank is reduced to zero in the contraction? To deal with this situation, it is standard to extend the definition of a graph to allow the presence of free loops, that is, edges with no endpoints. This terminology is due to Zaslavsky [23]. For a graph $G$ with free loops, the associated 2-polymatroid $M_{2}(G)$ is defined, as before, to have rank function $r(X)=|V(X)|$. The deletion of a free loop $f$ from a graph just removes $f$ from the graph. We define the contraction of $f$ to be the same as its deletion. For an edge $e$ that is not a free loop, to obtain a graph $H$ so that $M_{2}(G) / e=M_{2}(H)$, we let $H$ have edge set $E-e$ and vertex set $V-V(\{e\})$. An edge $x$ of $H$ is incident with the vertices in $V(\{x\})-V(\{e\})$.

The difference between $M_{2}(G) / e$ and $M_{2}(G / e)$ motivated us to introduce an operation for 2-polymatroids in [16] that mimics the effect of the usual operation of contraction of an edge from the graph.

Let $(E, r)$ be a 2-polymatroid $M$, and let $x$ be an element of $E$. We have described already what it means to add an element $x^{\prime}$ freely to $x$. Our new operation $M \downarrow x$ is obtained from $M$ by freely adding $x^{\prime}$ to $x$ in $M$, then contracting $x^{\prime}$ from the resulting extension, and finally deleting $x$. Because each of the steps in this process results in a 2-polymatroid, we have a well-defined operation on 2-polymatroids. When $x$ has rank at most one in $M$, one easily checks that $M \downarrow x=M / x$. When $x$ is a line in $M$, we see that $M \downarrow x$ and $M / x$ are different as their ranks are $r(M)-1$ and $r(M)-2$, respectively. Combining the different parts of the definition, we see that $M \downarrow x$ is the 2-polymatroid with ground set $E-\{x\}$ and rank function given, for all subsets $X$ of $E-\{x\}$, by

$$
r_{M \downarrow x}(X)= \begin{cases}r(X), & \text { if } r(\{x\})=0, \text { or } r(X \cup x)>r(X) ; \text { and } \\ r(X)-1, & \text { otherwise. }\end{cases}
$$

We shall say that $M \downarrow x$ has been obtained from $M$ by compressing $x$, and $M \downarrow x$ will be called the compression of $x$. We showed in [16] that $M_{2}(G) \downarrow e=M_{2}(G / e)$. Songbao Mo [13] established a number of properties of a generalization of this operation that he defines for connectivity functions and calls elision.

Instead of treating arbitrary minors, much of graph theory restricts attention to topological minors in which the only allowed contractions involve edges that meet vertices of degree two. When $e$ and $f$ are the only edges in a 2-connected graph $G$ meeting a vertex $v$, and $G$ has at least four vertices, $\{e, f\}$ is a 3-separating set in $M_{2}(G)$. This 3-separating set is an example of a special type of 3 -separating set that we introduced in [16]. In a 2-polymatroid $M$, a 3 -separating set $Z$ is prickly if it obeys the following conditions: 
(i) Each element of $Z$ is a line;

(ii) $|Z| \geqslant 2$ and $\lambda(Z)=2$;

(iii) $r\left((E-Z) \cup Z^{\prime}\right)=r(E-Z)+\left|Z^{\prime}\right|$ for all proper subsets $Z^{\prime}$ of $Z$; and

(iv) if $Z^{\prime}$ is a non-empty subset of $Z$, then

$$
r\left(Z^{\prime}\right)= \begin{cases}2 & \text { if }\left|Z^{\prime}\right|=1 ; \\ \left|Z^{\prime}\right|+2 & \text { if } 1<\left|Z^{\prime}\right|<|Z| ; \text { and } \\ |Z|+1 & \text { if }\left|Z^{\prime}\right|=|Z| .\end{cases}
$$

A prickly 3-separating set of $M$ will also be called a prickly 3 -separator of $M$. Observe that, when $Z$ is a prickly 3 -separating set, for all distinct $z$ and $z^{\prime}$ in $Z$, the 2-polymatroid $M \backslash z$ has $\left(\left\{z^{\prime}\right\}, E-\left\{z, z^{\prime}\right\}\right)$ as a 2-separation.

We are now able to formally state the main result of [16]. Recall that a 2-polymatroid is pure if every individual element has rank 2. It is non-empty if its ground set is non-empty.

Theorem 3. Let $M$ be a 3-connected non-empty 2-polymatroid. Then one of the following holds.

(i) $M$ has an element e such that $M \backslash e$ or $M / e$ is 3-connected;

(ii) $M$ has rank at least three and is a whirl or the cycle matroid of a wheel; or

(iii) $M$ is a pure 2-polymatroid having a prickly 3-separating set. Indeed, every minimal 3-separating set $Z$ with at least two elements is prickly, and $M \downarrow z$ is 3-connected and pure for all $z$ in $Z$.

In [16], we gave a number of examples to show the need for the third part of the above theorem. It is worth noting here, since it contrasts with what we have already mentioned and what will feature in the main result of this paper, the operation of deletion used in the last theorem does not incorporate compactification. In the main result of this paper, we will incorporate compactification as part of deletion but we will no longer need to allow arbitrary prickly compressions, only those that arise from a 2-element prickly 3-separator. These are precisely the 2-element subsets $Z$ of $E$ such that, for all non-empty subsets $Z^{\prime}$ of $Z$, we have $r\left(Z^{\prime}\right)=\left|Z^{\prime}\right|+1$ and $r\left(E-Z^{\prime}\right)=r(E)-\left|Z^{\prime}\right|+1$. Let $Z$ be such a set in a 2-polymatroid $M$. For $z$ in $Z$, we will call $M \downarrow z$ a series compression of $M$.

For a compact 2-polymatroid $M_{1}$, we call $M_{2}$ an s-minor of $M_{1}$ if $M_{2}$ can be obtained from $M_{1}$ by a sequence of contractions, deletions followed by compactifications, and series compressions. The next result is the main theorem of the paper. It concerns s-minors of 3-connected 2-polymatroids. Such a 2-polymatroid is compact provided it has at least three elements.

Theorem 4. Let $M$ be a 3-connected 2-polymatroid and $N$ be a 3-connected proper sminor of $M$ having at least four elements. Then one of the following holds. 
(i) $M$ has an element e such that $M /$ e is 3-connected having an s-minor isomorphic to $N$; or

(ii) $M$ has an element $e$ such that $(M \backslash e)^{b}$ is 3-connected having an s-minor isomorphic to $N$; or

(iii) $M$ has a two-element prickly 3-separating set $Z$ such that, for each $z$ in $Z$, the series compression $M \downarrow z$ is 3-connected having an s-minor isomorphic to $N$; or

(iv) $r(M) \geqslant 3$ and $M$ is a whirl or the cycle matroid of a wheel.

For compact 2-polymatroids $M_{1}$ and $M_{2}$, we call $M_{2}$ a $c$-minor of $M_{1}$ if $M_{2}$ can be obtained from $M_{1}$ by a sequence of operations each consisting of a contraction or of a deletion followed by a compactification. As we shall show in Section 6, the last theorem can be proved by establishing the following result.

Theorem 5. Let $M$ be a 3-connected 2-polymatroid and $N$ be a 3-connected proper cminor of $M$ having at least four elements. Then one of the following holds.

(i) $M$ has an element e such that $M / e$ is 3-connected having a c-minor isomorphic to $N$; or

(ii) $M$ has an element e such that $(M \backslash e)^{b}$ is 3-connected having a c-minor isomorphic to $N$; or

(iii) $M$ has a prickly 3-separator $\{y, z\}$ such that each of $M \downarrow y$ and $M \downarrow z$ is 3-connected having a c-minor isomorphic to $N$; or

(iv) $r(M) \geqslant 3$ and $M$ is a whirl or the cycle matroid of a wheel.

The paper is structured as follows. The next section includes some basic preliminaries. In Sections 3 and 4, we develop a number of results relating to connectivity and local connectivity, and to parallel connection and 2-sums. In Section 5, we describe the strategy for proving Theorem 4. That section serves as a guide to the remaining sections of the paper, with the purpose of each of these sections being to complete an identified step in the proof. Section 6 plays an important role in this proof by showing that the main theorem can be proved by adding the assumption that all series compressions are performed last in the production of an s-minor of $M$ isomorphic to $N$. That result is helpful but it cannot obscure the fact that the proof of Theorem 4 is complex with some subtleties in the logic that need to be carefully negotiated.

\section{Preliminaries}

Much of the terminology for matroids carries over to 2-polymatroids. For example, suppose $x$ and $y$ are distinct points of a 2-polymatroid $M$, that is, $r(\{x\})=1=r(\{y\})$. If $r(\{x, y\})=1$, then $x$ and $y$ are parallel points of $M$. On the other hand, if $r(E-\{x, y\})=$ 
$r(E)-1<r(E-x)=r(E-y)$, then $\{x, y\}$ is a series pair of points of $M$. Evidently, if $\{x, y\}$ is a parallel or series pair of points, then $\lambda_{M}(\{x, y\}) \leqslant 1$. If $x$ and $y$ are distinct lines of $M$ and $r(\{x, y\})=2$, then $x$ and $y$ are parallel lines of $M$. If $x$ is a point and $y$ is a line of $M$, then $x$ lies on $y$ if $r(\{x, y\})=2$.

One tool that is used repeatedly in our earlier work is the submodularity of the connectivity function. Once again, this will play a vital role here. Partitions $\left(X_{1}, X_{2}\right)$ and $\left(Y_{1}, Y_{2}\right)$ of a set $E$ are said to cross if all four of the sets $X_{1} \cap Y_{1}, X_{1} \cap Y_{2}, X_{2} \cap Y_{1}$, and $X_{2} \cap Y_{2}$ are non-empty. We shall frequently encounter crossing partitions of the ground set of a 2-polymatroid.

In this paper, we shall frequently switch between considering the deletion $M \backslash X$ of a set $X$ of elements of a 2-polymatroid $M$ and the compactification $(M \backslash X)^{b}$ of this deletion, which we shall sometimes call the compactified deletion of $X$. We shall often use the following abbreviated notation for the latter:

$$
(M \backslash X)^{b}=M \rrbracket X .
$$

We shall often encounter the situation when we have a 2-polymatroid $M$ such that $M^{b}$ is 3-connected although $M$ itself is not. This occurs when $M$ has a line $\ell$ such that $(\{\ell\}, E-\ell)$ is a 2-separation. We call such a 2-separation of $M$ trivial. Thus, in general, a partition $(X, Y)$ of $E$ is a non-trivial 2-separation of $M$ if $\lambda_{M}(X) \leqslant 1$ and $\min \{|X|,|Y|\} \geqslant 2$.

For a 2-polymatroid $M$, we recall that a minor of $M$ is any 2-polymatroid that can be obtained from $M$ by a sequence of contractions and deletions where, here, deletions are not automatically accompanied by compactifications. When $M$ and $N$ are compact, we defined $N$ to be a c-minor of $M$ if it can be obtained from $M$ by a sequence of contractions and deletions followed by compactifications. In the proof of Theorem 5 , it is convenient to be able to separate the compactifications from the deletions. Thus we define a $c$-minor of an arbitrary 2-polymatroid $M$ to be any 2-polymatroid that can be obtained from $M$ by a sequence of contractions, deletions, and compactifications. As we shall show in Corollary 9, this extension of the definition is consistent with our original definition. For a 2-polymatroid $N$, a special $N$-minor of $M$ is any c-minor of $M$ that is either equal to $N$ or differs from $N$ by having a single point relabelled.

Lemma 6. Let $P$ and $Q$ be 2-polymatroids such that $Q$ can be obtained from $P$ by a sequence of deletions, contractions, and compactifications with the last move being a compactification. Then $Q$ can be obtained from $P$ by the same sequence of deletions and contractions with none of the compactifications being done except for the last move.

To prove this lemma, we shall require a preliminary result.

Lemma 7. Let $P$ be the 2-polymatroid $(E, r)$. For $A \subseteq E$,

(i) $\left(P^{b} \backslash A\right)^{b}=(P \backslash A)^{b}$; and

(ii) $\left(P^{b} / A\right)^{b}=(P / A)^{b}$. 
Proof. Let $P_{1}$ be a 2-polymatroid with ground set $E$ and rank function $r_{1}$. Then, for $X \subseteq E-A$, we have

$$
\begin{aligned}
r_{\left(P_{1} \backslash A\right)^{b}}(X) & =r_{P_{1} \backslash A}(X)+\sum_{x \in X}\left[\lambda_{P_{1} \backslash A}(\{x\})-r_{P_{1} \backslash A}(\{x\})\right] \\
& =r_{1}(X)+\sum_{x \in X}\left[r_{1}(E-A-x)-r_{1}(E-A)\right] .
\end{aligned}
$$

Thus

$$
r_{(P \backslash A)^{b}}(X)=r(X)+\sum_{x \in X}[r(E-A-x)-r(E-A)] .
$$

Next we observe that, for $x$ in $X$,

$$
\begin{aligned}
r_{P^{b}}(E-A-x)-r_{P^{b}}(E-A)= & r(E-A-x)+\sum_{y \in E-A-x}[\lambda(\{y\})-r(\{y\})] \\
& -r(E-A)-\sum_{y \in E-A}[\lambda(\{y\})-r(\{y\})] \\
& =r(E-A-x)-r(E-A)-\lambda(\{x\})+r(\{x\}) .
\end{aligned}
$$

Thus, by (2), (4), and (3),

$$
\begin{aligned}
r_{\left(P^{b} \backslash A\right)^{b}}(X) & =r_{P^{b}}(X)+\sum_{x \in X}\left[r_{P^{b}}(E-A-x)-r_{P^{b}}(E-A)\right] \\
& =r(X)+\sum_{x \in X}[\lambda(\{x\})-r(\{x\})+r(E-A-x)-r(E-A) \\
& =r(X)+\sum_{x \in X}[r(E-A-x)-r(E-A)] \\
& =r_{(P \backslash A)^{b}}(X) .
\end{aligned}
$$

We conclude that (i) holds.

Again, for $X \subseteq E-A$, we have

$$
\begin{aligned}
r_{\left(P_{1} / A\right)^{b}}(X) & =r_{P_{1} / A}(X)+\sum_{x \in X}\left[\lambda_{P_{1} / A}(\{x\})-r_{P_{1} / A}(\{x\})\right] \\
& =r_{1}(X \cup A)-r_{1}(A)+\sum_{x \in X}\left[r_{P_{1} / A}(E-A-x)-r_{P_{1} / A}(E-A)\right] \\
& =r_{1}(X \cup A)-r_{1}(A)+\sum_{x \in X}\left[r_{1}(E-x)-r_{1}(E)\right] .
\end{aligned}
$$

Thus

$$
r_{(P / A)^{b}}(X)=r(X \cup A)-r(A)+\sum_{x \in X}[r(E-x)-r(E)]
$$


Therefore, by (5), (4), and (6)

$$
\begin{aligned}
r_{\left(P^{b} / A\right)^{b}}(X) & =r_{P^{b}}(X \cup A)-r_{P^{b}}(A)+\sum_{x \in X}\left[r_{P^{b}}(E-x)-r_{P^{b}}(E)\right] \\
& =r(X \cup A)-r(A)+\sum_{x \in X}[\lambda(\{x\})-r(\{x\})+r(E-x)-r(E) \\
& =r(X \cup A)-r(A)+\sum_{x \in X}[r(E-x)-r(E)] \\
& =r_{(P / A)^{b}}(X) .
\end{aligned}
$$

Hence (ii) holds.

Proof of Lemma 6. We may assume that there are disjoint subsets $A_{1}, A_{2}, \ldots, A_{n}$ of $E$ such that, in forming $Q$ from $P$, these sets are removed in order via deletion or contraction with the possibility that, after each such move, a compactification is performed. To prove the lemma, we argue by induction on $n$. It follows immediately from Lemma 7 that the lemma holds if $n=1$. Assume the result holds for $n<m$ and let $n=m \geqslant 2$. Then there is a 2-polymatroid $R$ such that $Q$ is $\left(R \backslash A_{n}\right)^{b}$ or $\left(R / A_{n}\right)^{b}$, so, by Lemma 7, $Q$ is $\left(R^{b} \backslash A_{n}\right)^{b}$ or $\left(R^{b} / A_{n}\right)^{b}$, respectively. In forming $R$, a certain sequence of deletions, contractions, and compactifications is performed. Let $R_{0}$ be the 2-polymatroid that is obtained from $P$ by performing the same sequence of operations except for the compactifications. Then, by the induction assumption, $R^{b}=R_{0}^{b}$. Since $\left(R^{b} \backslash A_{n}\right)^{b}=\left(R_{0}^{b} \backslash A_{n}\right)^{b}=\left(R_{0} \backslash A_{n}\right)^{b}$ and $\left(R^{b} / A_{n}\right)^{b}=\left(R_{0}^{b} / A_{n}\right)^{b}=\left(R_{0} / A_{n}\right)^{b}$, the lemma follows by induction.

The following are straightforward consequences of Lemma 6 . We prove only the second of these.

Corollary 8. Let $P$ and $Q$ be 2-polymatroids such that $Q$ is compact. Then $Q$ is a c-minor of $P$ if and only if $Q$ can be obtained from $P$ by a sequence of deletions and contractions followed by a single compactification.

Corollary 9. Let $P$ and $Q$ be compact 2-polymatroids. Then $Q$ is a c-minor of $P$ if and only if $Q$ can be obtained from $P$ by a sequence of operations each of which consists of either a contraction or a deletion followed by a compactification.

Proof. We need to show that if $Q$ is a c-minor of $P$, then $Q$ can be obtained as described. Now $Q^{b}=Q$. Thus, by Lemma $6, Q$ can be obtained from $P$ by a sequence of deletions and contractions with one compactification being done as the final move. By Lemma 7 , we can perform a compactification after each deletion and still obtain $Q$ at the end of the process. Since $P$ is compact and each contraction of a compact 2-polymatroid is compact, we retain compactness throughout this sequence of moves, so the result holds.

We will need some elementary properties of deletion, contraction, and series compression. 
Lemma 10. Let $A$ and $B$ be disjoint subsets of the ground set $E$ of a 2-polymatroid $P$. Then

(i) $P / A / B=P /(A \cup B)=P / B / A$;

(ii) $P \Downarrow A \Downarrow B=P \rrbracket(A \cup B)=P \Downarrow B \backslash A$; and

(iii) $P / A \rrbracket B=P \Downarrow B / A$.

Proof. Because the proofs of all three parts are routine, we only include a proof of (iii). Suppose $X \subseteq E-(A \cup B)$. Then

$$
\begin{aligned}
r_{P / A \Downarrow B}(X) & =r_{((P / A) \backslash B)^{b}}(X) \\
& =r_{P / A}(X)+\sum_{x \in X}\left[\lambda_{P / A \backslash B}(\{x\})-r_{P / A \backslash B}(\{x\})\right] \\
& =r_{P / A}(X)+\sum_{x \in X}\left[r_{P / A}(E-A-B-x)-r_{P / A}(E-A-B)\right] \\
& =r(X \cup A)-r(A)+\sum_{x \in X}[r(E-B-x)-r(E-B)] \\
& =r(X \cup A)-r(A)+\sum_{x \in X}\left[\lambda_{P \backslash B}(\{x\})-r_{P \backslash B}(\{x\})\right] \\
& =r_{P \Downarrow B}(X \cup A)-r_{P \Downarrow B}(A) \\
& =r_{P \Downarrow B / A}(X) .
\end{aligned}
$$

We conclude that (iii) holds.

Lemma 11. Let $M$ be a polymatroid. Then

$$
\left(M^{b}\right)^{*}=M^{*}=\left(M^{*}\right)^{b} .
$$

Proof. By Lemma 2(i), $M^{*}$ is compact, so $M^{*}=\left(M^{*}\right)^{b}$. Also, by Lemma 2(ii), $\left(M^{b}\right)^{*}=$ $\left(\left(M^{*}\right)^{*}\right)^{*}=\left(M^{*}\right)^{b}$.

Lemma 12. Let $P$ and $Q$ be 2-polymatroids, where $Q$ is compact. Then $P$ has a c-minor isomorphic to $Q$ if and only if $P^{*}$ has a c-minor isomorphic to $Q^{*}$.

Proof. Suppose $P$ has a c-minor isomorphic to $Q$. By Corollary $8, Q$ can be obtained from $P$ by a sequence of deletions and contractions with one compactification being done as the final move. By Lemma 7, we can perform a compactification after each deletion and after each contraction and still obtain $Q$ at the end of the process. Indeed, since $\left(P^{b} \backslash A\right)^{b}=(P \backslash A)^{b}$ and $\left(P^{b} / A\right)^{b}=(P / A)^{b}$, we see that $P^{b}$ has a c-minor isomorphic to $Q$. Thus we may assume that, in forming $Q$ from $P^{b}$, we remove, in order, disjoint sets $A_{1}, A_{2}, \ldots, A_{n}$ where each such removal is followed by a compactification. To prove that $P^{*}$ has a c-minor isomorphic to $Q^{*}$, we shall argue by induction on $n$. 
Suppose $n=1$. Then $Q$ is $\left(P^{b} \backslash A_{1}\right)^{b}$ or $\left(P^{b} / A_{1}\right)^{b}$. Then, by Lemmas 2 and 11 ,

$$
\left(\left(P^{b} \backslash A_{1}\right)^{b}\right)^{*}=\left(\left(\left(P^{*}\right)^{*} \backslash A_{1}\right)^{b}\right)^{*}=\left(\left(P^{*} / A_{1}\right)^{*}\right)^{*}=\left(P^{*} / A_{1}\right)^{b}=P^{*} / A_{1}
$$

and

$$
\left(\left(P^{b} / A_{1}\right)^{b}\right)^{*}=\left(P^{b} / A_{1}\right)^{*}=\left(\left(P^{b}\right)^{*} \backslash A_{1}\right)^{b}=\left(P^{*} \backslash A_{1}\right)^{b} .
$$

Since $Q$ is compact, we deduce that the result holds for $n=1$. Assume it holds for $n<k$ and let $n=k \geqslant 2$. Then there is a compact 2-polymatroid $R$ that is a c-minor of $P$ such that $Q$ is $\left(R \backslash A_{n}\right)^{b}$ or $\left(R / A_{n}\right)^{b}$. By the induction assumption, $R^{*}$ is a c-minor of $P^{*}$, and $Q^{*}$ is a c-minor of $R^{*}$. Hence $Q^{*}$ is a c-minor of $P^{*}$.

For the converse, we note that, by what we have just proved, if $Q^{*}$ is a c-minor of $P^{*}$, then $\left(Q^{*}\right)^{*}$ is a c-minor of $\left(P^{*}\right)^{*}$, that is, $Q^{b}$ is a c-minor of $P^{b}$. But $Q$ is compact so $Q$ is a c-minor of $P^{b}$. Hence $Q$ is a c-minor of $P$.

When we compactify a 2-polymatroid, loosely speaking what we are doing is dealing simultaneously with a number of 2 -separations. It will be helpful to be able to treat these 2 -separations one at a time. In the introduction, we defined the compression $M \downarrow x$ for an element $x$ of a 2-polymatroid $M$. Ultimately, that operation removes $x$. Let $M \downarrow x$ be the 2-polymatroid that is obtained from $M$ by freely adding an element $x^{\prime}$ on $x$ and then contracting $x^{\prime}$. Thus $M \downarrow \underline{\downarrow}$ has ground set $E$ and rank function given, for all subsets $X$ of $E$, by

$$
r_{M_{\downarrow} x}(X)= \begin{cases}r(X), & \text { if } r(\{x\})=0, \text { or } r(X \cup x)>r(X) ; \text { and } \\ r(X)-1, & \text { otherwise. }\end{cases}
$$

We shall say that $M \downarrow x$ has been obtained by compactifying $x$. Evidently

$$
M \downarrow x=(M \underline{\downarrow} x) \backslash x .
$$

Lemma 13. Let $M$ be a 2-connected 2-polymatroid that is not compact. Let $Z$ be the set of lines $z$ of $M$ such that $\lambda(\{z\})=1$. Then

$$
M^{b}=\left(\left(\ldots\left(\left(M \downarrow z_{1}\right) \downarrow z_{2}\right) \ldots\right) \downarrow z_{n}\right)
$$

where $Z=\left\{z_{1}, z_{2}, \ldots, z_{n}\right\}$.

Proof. We argue by induction on $n$. Suppose $n=1$. Let $X \subseteq E(M)$. Then

$$
r_{M^{b}}(X)= \begin{cases}r(X), & \text { if } z_{1} \notin X ; \text { and } \\ r(X)-1, & \text { otherwise }\end{cases}
$$

On the other hand,

$$
r_{M_{\downarrow} z_{1}}(X)= \begin{cases}r(X), & \text { if } r\left(X \cup z_{1}\right)>r(X) ; \text { and } \\ r(X)-1, & \text { otherwise. }\end{cases}
$$

The result is easily checked in this case. 
Now assume that $n \geqslant 2$ and that the lemma holds if $|Z| \leqslant n-1$. Let $M_{1}=M \downarrow z_{1}$. Then $M_{1}$ is easily shown to be 2 -connected having $\left\{z_{2}, z_{3}, \ldots, z_{n}\right\}$ as its set of lines $z$ for which $\lambda_{M_{1}}(\{z\})=1$. Thus, by the induction assumption,

$$
M_{1}^{b}=\left(\left(\ldots\left(\left(M_{1} \downarrow z_{2}\right) \downarrow z_{3}\right) \ldots\right) \downarrow z_{n}\right) .
$$

Since $M_{1}=M \downarrow z_{1}$, it suffices to show that $M_{1}^{\mathrm{b}}=M^{\mathrm{b}}$.

Suppose $X \subseteq E$. Then

$$
r^{b}(X)=r(X)+\sum_{x \in X}(\lambda(\{x\})-r(\{x\})) .
$$

Now

$$
r_{M_{1}}(X)= \begin{cases}r(X), & \text { if } r\left(X \cup z_{1}\right)>r(X) ; \text { and } \\ r(X)-1, & \text { otherwise }\end{cases}
$$

Thus

$$
r_{M_{1}}(X)= \begin{cases}r(X), & \text { if } z_{1} \notin X ; \text { and } \\ r(X)-1, & \text { otherwise. }\end{cases}
$$

Hence

$$
\begin{aligned}
r_{M_{1}^{b}}(X) & =r_{M_{1}}(X)+\sum_{x \in X}\left(\lambda_{M_{1}}(\{x\})-r_{M_{1}}(\{x\})\right) \\
& =r_{M_{1}}(X)+\sum_{x \in X \cap\left(Z-z_{1}\right)}\left(\lambda_{M}(\{x\})-r_{M}(\{x\})\right) \\
& =r_{M}(X)+\sum_{x \in X \cap Z}\left(\lambda_{M}(\{x\})-r_{M}(\{x\})\right) \\
& =r^{b}(X) .
\end{aligned}
$$

We conclude, by induction, that the lemma holds.

The remainder of this section presents a number of basic properties of 2-element prickly 3 -separators and of the compression operation.

Lemma 14. Let $P$ be a 2-polymatroid having $j$ and $k$ as lines and with $r(\{j, k\})=3$. Suppose $X \subseteq E(P)-k$ and $j \in X$. Then $r_{P \downarrow k}(X)=r(X \cup k)-1$.

Proof. By definition,

$$
r_{P \downarrow k}(X)= \begin{cases}r(X), & \text { if } r(X \cup k)>r(X) ; \\ r(X)-1, & \text { otherwise. }\end{cases}
$$

As $j \in X$ and $r(\{j, k\})=3$, by submodularity, $r(X \cup k)$ is $r(X)$ or $r(X)+1$. Thus $r_{P \downarrow k}(X)=r(X \cup k)-1$. 
Lemma 15. Let $P$ be a 2-polymatroid having $j$ and $k$ as lines and with $r(\{j, k\})=3$. Suppose $\ell$ is a line of $P$ that is not in $\{j, k\}$ and is not parallel to $k$. Then $\{\ell\}$ is 2separating in $P$ if and only if it is 2-separating in $P \downarrow k$.

Proof. Clearly $\{\ell\}$ is 2-separating in $P$ if and only if $r(E-\ell) \leqslant r(E)-1$. Since $\ell$ is not parallel to $k$, we see that $r_{P \downarrow k}(\ell)=r(\ell)=2$. Now $\{\ell\}$ is 2-separating in $P \downarrow k$ if and only if $r_{P \downarrow k}(E-\{k, \ell\}) \leqslant r_{P \downarrow k}(E-k)-1$. By Lemma 14, the last inequality holds if and only if $r(E-\ell)-1 \leqslant r(E)-1-1$. We conclude that the lemma holds.

Lemma 16. Let $\{j, k\}$ be a prickly 3-separator in a 2-polymatroid $P$. Then $P \downarrow j$ can be obtained from $P \downarrow k$ by relabelling $j$ as $k$.

Proof. Suppose $X \subseteq E-\{j, k\}$. Then, since both $r(X \cup j)$ and $r(X \cup k)$ exceed $r(X)$,

$$
r_{P \downarrow j}(X)=r_{P}(X)=r_{P \downarrow k}(X) .
$$

As $r(\{j, k\})=3$, submodularity implies that either $r(X \cup j \cup k)=r(X \cup j)+1$, or $r(X \cup j \cup k)=r(X \cup j)$. Thus $r_{P \downarrow k}(X \cup j)=r(X \cup j \cup k)-1$. By symmetry, $r_{P \downarrow j}(X \cup k)=$ $r(X \cup j \cup k)-1$, and the lemma follows.

Lemma 17. Let $P$ be a compact 2-polymatroid and $\{j, k\}$ be a prickly 3-separator of $P$. Then $P \downarrow k$ is compact.

Proof. We prove (ii). It suffices to show that $r_{P \downarrow k}(E-k-y)=r_{P \downarrow k}(E-k)$ for all $y$ in $E-k$. Since $P$ is compact, $r(E-k)=r(E)$, so $r_{P \downarrow k}(E-k)=r(E)-1$. Now

$$
r_{P \downarrow k}(E-k-y)= \begin{cases}r(E-k-y), & \text { if } r(E-y)-1 \geqslant r(E-y-k) ; \text { and } \\ r(E-k-y)-1, & \text { otherwise. }\end{cases}
$$

It follows that $r_{P \downarrow k}(E-k-y)=r(E)-1=r_{P \downarrow k}(E-k)$ unless $r(E-k-y)=r(E-y)-2$. Consider the exceptional case. Evidently $y \neq j$ as $r(E-k-j)=r(E-j)-1$. Thus $j \in E-k-y$. Since $\Pi(\{j\},\{k\})=1$, it follows that $r(E-y) \leqslant r(E-k-y)+1$. This contradiction completes the proof of (ii).

Lemma 18. In a 2-polymatroid $P$, let $k$ and $y$ be distinct elements. Then

(i) $P \downarrow k \backslash y=P \backslash y \downarrow k$; and

(ii) $P \downarrow k / y=P / y \downarrow k$.

Proof. Part (i) is essentially immediate. We now prove (ii). If $r(\{k\}) \leqslant 1$, then $P \downarrow k=$ $P / k$, so

$$
P \downarrow k / y=P / k / y=P / y / k=P / y \downarrow k .
$$

Thus we may assume that $r(\{k\})=2$.

Suppose $y$ is a line such that $r(\{y, k\})=2$. Then

$$
P / y \downarrow k=P / y / k=P / k / y=P \downarrow k / y
$$


where the last equality follows by considering how $P \downarrow k$ is constructed. Thus we may assume that $y$ is not a line that is parallel to $k$. Hence

$$
r(\{y, k\})>r(\{y\}) .
$$

Let $X$ be a subset of $E-k-y$. Then

$$
r_{P \downarrow k / y}(X)=r_{P \downarrow k}(X \cup y)-r_{P \downarrow k}(\{y\})=r_{P \downarrow k}(X \cup y)-r(\{y\})
$$

where the second equality follows because $r(\{y, k\})>r(\{y\})$. We deduce that

$$
r_{P \downarrow k / y}(X)= \begin{cases}r(X \cup y)-r(\{y\}), & \text { if } r(X \cup y \cup k)>r(X \cup y) ; \\ r(X \cup y)-r(\{y\})-1, & \text { otherwise. }\end{cases}
$$

On the other hand, since $r_{P / y}(X \cup k)=r(X \cup k \cup y)-r(\{y\})$ and $r_{P / y}(X)=r(X \cup y)-$ $r(\{y\})$, we see that

$$
r_{P / y \downarrow k}(X)= \begin{cases}r(X \cup y)-r(\{y\}), & \text { if } r(X \cup y \cup k)>r(X \cup y) ; \\ r(X \cup y)-r(\{y\})-1, & \text { otherwise. }\end{cases}
$$

Thus

$$
r_{P \downarrow k / y}(X)=r_{P / y \downarrow k}(X)
$$

so the lemma holds.

Lemma 19. Let $\{j, k\}$ be a prickly 3-separator in a 2-polymatroid $P$. If $P \downarrow k$ is 3connected, then so is $P$ unless $|E|=4$ and $r(P)=3$ where each of $j$ and $k$ has a single point on it.

Proof. Let $(X, Y)$ be an exact $m$-separation of $P$ for some $m$ in $\{1,2\}$ where $k \in X$. Then $r(X)+r(Y)-r(P)=m-1$. Now $r(P \downarrow k)=r(P)-1$.

Consider $r_{P \downarrow k}(X-k)+r_{P \downarrow k}(Y)$. Suppose first that $j \in X-k$. Then, by Lemma 14, $r_{P \downarrow k}(X-k)=r(X)-1$ and $r_{P \downarrow k}(Y)=r(Y)$. Hence

$$
r_{P \downarrow k}(X-k)+r_{P \downarrow k}(Y)-r(P \downarrow k)=m-1 .
$$

As $P \downarrow k$ is 2-connected, we cannot have $m=1$ since both $X-k$ and $Y$ are non-empty. Thus $m=2$ so $r(X)+r(Y)-r(P)=1$. Now $\max \{|X|, r(X)\} \geqslant 2$ and $\max \{|Y|, r(Y)\} \geqslant$ 2. Thus $\max \left\{|Y|, r_{P \downarrow k}(Y)\right\} \geqslant 2$. If $X=\{j, k\}$, then, as $\{j, k\}$ is a 3-separator of $P$, we have $r(X)+r(Y)-r(P)=2$, a contradiction. We deduce that $|X-k| \geqslant 2$, so $(X-k, Y)$ is a 2-separation of $P \downarrow k$, a contradiction.

We may now assume that $j \in Y$. Note that $X-k$ is non-empty otherwise $m-1=$ $r(\{k\})+r(E-k)-r(P)=2$, a contradiction. We have

$$
r_{P \downarrow k}(X-k)+r_{P \downarrow k}(Y)-r(P \downarrow k) \leqslant(r(X)-1)+r(Y)-(r(P)-1)=m-1 .
$$


As $P \downarrow k$ is 2-connected, $m=2$ and equality holds throughout. We deduce that

$$
r_{P \downarrow k}(X-k)=r(X)-1,
$$

and

$$
r_{P \downarrow k}(Y)=r(Y) .
$$

Moreover, $\max \left\{r_{P \downarrow k}(X-k),|X-k|\right\} \leqslant 1$. As $X-k$ is non-empty,

$$
\max \left\{r_{P \downarrow k}(X-k),|X-k|\right\}=1 .
$$

Let $X-k=\{x\}$. Then $r_{P \downarrow k}(\{x\}) \leqslant 1$. Now

$$
r_{P \downarrow k}(\{x\})= \begin{cases}r(\{x\}) & \text { if } r(\{x, k\})>r(\{x\}) ; \\ r(\{x\})-1 & \text { if } r(\{x, k\})=r(\{x\}) .\end{cases}
$$

Suppose $r(\{x, k\})=r(\{x\})$. Then $x$ and $k$ are parallel lines. Thus $r(E)=r(E-j)=$ $r(E-\{j, k\})=r(E)-1$, a contradiction. We deduce that $r(\{x, k\})>r(\{x\})$. Hence $1 \geqslant$ $r_{P \downarrow k}(\{x\})=r(\{x\})$. But, by (8), $r_{P \downarrow k}(\{x\})=r(\{x, k\})-1$, so $r(\{x, k\})=r(\{x\})+1 \geqslant 2$. Thus $r(\{x\})=1$ and $r(\{x, k\})=2$, so $r(Y)=r(P)-1$. As $r(Y \cup x)=r(E-k)=r(P)$, we deduce that $r(\{j, x\})=3$.

Now $Y-j \neq \emptyset$ otherwise $E=\{j, k, x\}$ and $r(\{j, k\})+r(\{x\})-r(E)=1$, so $\{j, k\}$ is not a 3-separator, a contradiction. Since $r(\{j, x, k\})=3=r(\{j, x\})$, we have

$$
r_{P \downarrow k}(\{j, x\})=r(\{j, x\})-1=2 .
$$

By $(9), r_{P \downarrow k}(Y)=r(Y)$. Thus $r(Y \cup k)>r(Y)$, so $r((Y-j) \cup k)>r(Y-j)$ and $r_{P \downarrow k}(Y-j)=r(Y-j)$. Also $r(Y-j)<r(Y)$ so $r(Y-j) \leqslant r(P)-2$. Thus

$$
\begin{aligned}
r_{P \downarrow k}(\{j, x\})+r_{P \downarrow k}(Y-j)-r(P \downarrow k) & =2+r(Y-j)-r(P)+1 \\
& \leqslant 2+(r(P)-2)-r(P)+1 \\
& =1 .
\end{aligned}
$$

As $P \downarrow k$ is 3-connected and $Y-j$ is non-empty, equality must hold here and $\max \left\{r_{P \downarrow k}(Y-\right.$ $j),|Y-j|\}=1$.

Let $Y-j=\{y\}$. Then $r_{P \downarrow k}(\{y\})=r(\{y\})=1$ and $r(\{y\})=r(P)-2$, so $r(P)=3$. Moreover, $r(\{j, y\})=r(Y)=r(P)+1-r(X)=2$. As $P \downarrow k$ is 3-connected, $y$ and $x$ are not parallel points in $P \downarrow k$, so $y$ does not lie on $k$. We conclude that $P$ has rank 3 and consists of two lines, $j$ and $k$, and two points, $y$ and $x$, where $y$ is on $j$ but not $k$, and $x$ is on $k$ but not $j$. Thus the lemma holds.

\section{Some results for connectivity and local connectivity}

This section notes a number of properties of the connectivity and local-connectivity functions that will be used in the proof of the main theorem. First we show that compression 
is, in most situations, a self-dual operation. We proved this result in [16, Proposition 3.1] for the variant of duality used there. By making the obvious replacements in that proof, it is straightforward to check that the result holds with the modified definition of duality used here. We omit the details.

Proposition 20. Let e be a line of a 2-polymatroid $M$ and suppose that $M$ contains no line parallel to e. Then

$$
M^{*} \downarrow e=(M \downarrow e)^{*} .
$$

The next proposition implies that Theorems 4 and 5 are self-dual results. Its proof will use the following result.

Lemma 21. The set $\{j, k\}$ is a prickly 3-separator of a 2-polymatroid $M$ if and only if it is a prickly 3-separator in $M^{*}$.

Proof. Suppose $\{j, k\}$ is a prickly 3 -separator of $M$. By Lemma 2,

$$
\lambda_{M^{*}}(\{j, k\})=\lambda_{M}(\{j, k\})=2 .
$$

Moreover, it is straightforward to check that $r_{M^{*}}(\{j\})=2=r_{M^{*}}(\{k\})$, that $r_{M^{*}}(\{j, k\})=$ 3 , and that $\Pi_{M^{*}}(\{j\}, E-\{j, k\})=1=\Pi_{M^{*}}(\{k\}, E-\{j, k\})$. Hence $\{j, k\}$ is a prickly 3 -separator of $M^{*}$. Conversely, suppose that $\{j, k\}$ is a prickly 3 -separator of $M^{*}$. Then, by what we have just shown, $\{j, k\}$ is a prickly 3 -separator of $\left(M^{*}\right)^{*}$, that is, of $M^{b}$. Now $2=\lambda_{M^{b}}(\{j, k\})=\lambda_{M}(\{j, k\})$. Moreover, since $r_{M^{b}}(\{j\})=2$, it follows that $\lambda(\{j\})=2$, so $r(\{j\})=2$ and $r(E-j)=r(E)$. Similarly, $\lambda(\{k\})=2=r(\{k\})$ and $r(E-k)=r(E)$. It follows, since $r_{M^{b}}(\{j, k\})=3$, that $r(\{j, k\})=3$. By using the fact that $\sqcap_{M^{b}}(\{j\}, E-$ $\{j, k\})=1=\sqcap_{M^{b}}(\{k\}, E-\{j, k\})$, it is not difficult to check that $\sqcap(\{j\}, E-\{j, k\})=$ $1=\sqcap(\{k\}, E-\{j, k\})$. We conclude that $\{j, k\}$ is a prickly 3 -separator of $M$, so the lemma holds.

Proposition 22. Let $P$ and $Q$ be compact 2-polymatroids. Then

(i) $Q$ is an c-minor of $P$ if and only if $Q^{*}$ is an c-minor of $P^{*}$; and

(ii) $Q$ is an s-minor of $P$ if and only if $Q^{*}$ is an s-minor of $P^{*}$.

Proof. By Lemma 2, both $P^{*}$ and $Q^{*}$ are compact. Moreover, $\left(P^{*}\right)^{*}=P$ and $\left(Q^{*}\right)^{*}=Q$. By Lemma 2 again, for an element $\ell$ of $P$, we have that $(P \Downarrow \ell)^{*}=P^{*} / \ell$ and $(P / \ell)^{*}=P^{*} \| \ell$. Part (i) now follows by a straightforward induction argument. To prove (ii), assume $Q$ is an s-minor of $P$. It suffices to show that $Q^{*}$ is an s-minor of $P^{*}$. If $\{j, k\}$ is a prickly 3 -separator of $P$, then, by Lemma 21 , it is a prickly 3 -separator of $P^{*}$. By Lemma 17 , $P \downarrow k$ is compact and, by Proposition 20, $(P \downarrow k)^{*}=P^{*} \downarrow k$. This and the argument for (i) give that the dual of each allowable move on $P$ produces a 2-polymatroid that is obtained from $P^{*}$ by an allowable move. Another straightforward induction argument gives (ii). 
Let $M$ be a polymatroid $(E, r)$. If $X$ and $Y$ are subsets of $E$, the local connectivity $\sqcap(X, Y)$ between $X$ and $Y$ is defined by $\sqcap(X, Y)=r(X)+r(Y)-r(X \cup Y)$. Sometimes we will write $\Pi_{M}$ for $\sqcap$, and $\Pi^{*}$ for $\sqcap_{M^{*}}$. It is straightforward to prove the following. Again this holds for both the version of duality used here and the variant used in [16].

Lemma 23. Let $M$ be a polymatroid $(E, r)$. For disjoint subsets $X$ and $Y$ of $E$,

$$
\sqcap_{M^{*}}(X, Y)=\sqcap_{M /(E-(X \cup Y))}(X, Y) .
$$

The next lemma will be used repeatedly, often without explicit reference. Two sets $X$ and $Y$ in a polymatroid $M$ are skew if $\sqcap(X, Y)=0$.

Lemma 24. Let $M$ be a 2-polymatroid and $z$ be an element of $M$ such that $(A, B)$ is a 2-separation of $M / z$. Suppose $z$ is skew to $A$. Then $(A, B \cup z)$ is a 2-separation of $M$. Moreover, if $M$ is 3-connected, then $A$ is not a single line in $M / z$.

Proof. Clearly $r(A \cup z)-r(\{z\})=r(A)$, so $(A, B \cup z)$ is a 2-separation of $M$. If $M$ is 3 -connected and $A$ consists of a single line $a$ of $M / z$, then $a$ is a line of $M$, so $a$ and $z$ are skew, and we obtain the contradiction that $M$ has a 2-separation.

Numerous properties of the connectivity function of a matroid are proved simply by applying properties of the rank function; they do not rely on the requirement that $r(\{e\}) \leqslant$ 1 for all elements $e$. Evidently, such properties also hold for the connectivity function of a polymatroid. The next few lemmas note some of these properties.

The first two are proved in [14, Lemmas 8.2.3 and 8.2.4].

Lemma 25. Let $(E, r)$ be a polymatroid and let $X_{1}, X_{2}, Y_{1}$, and $Y_{2}$ be subsets of $E$ with $Y_{1} \subseteq X_{1}$ and $Y_{2} \subseteq X_{2}$. Then

$$
\sqcap\left(Y_{1}, Y_{2}\right) \leqslant \sqcap\left(X_{1}, X_{2}\right)
$$

Lemma 26. Let $(E, r)$ be a polymatroid $M$ and let $X, C$, and $D$ be disjoint subsets of $E$. Then

$$
\lambda_{M \backslash D / C}(X) \leqslant \lambda_{M}(X) .
$$

Moreover, equality holds if and only if

$$
r(X \cup C)=r(X)+r(C)
$$

and

$$
r(E-X)+r(E-D)=r(E)+r(E-(X \cup D)) .
$$

The following [14, Corollary 8.7.6] is a straightforward consequence of the last lemma.

Corollary 27. Let $X$ and $D$ be disjoint subsets of the ground set $E$ of a polymatroid $M$. Suppose that $r(M \backslash D)=r(M)$. Then

(i) $\lambda_{M \backslash D}(X)=\lambda_{M}(X)$ if and only if $D \subseteq \mathrm{cl}_{M}(E-(X \cup D))$; and 
(ii) $\lambda_{M \backslash D}(X)=\lambda_{M}(X \cup D)$ if and only if $D \subseteq \operatorname{cl}_{M}(X)$.

It is well known that, when $M$ is a matroid, for all subsets $X$ of $E(M)$,

$$
\lambda_{M}(X)=r_{M}(X)+r_{M^{*}}(X)-|X| .
$$

It is easy to check that the following variant on this holds for polymatroids. Recall that $\|X\|=\sum_{x \in X} r(\{x\})$.

Lemma 28. In a polymatroid $M$, for all subsets $X$ of $E(M)$,

$$
\lambda_{M}(X)=r_{M}(X)+r_{M^{*}}(X)-\|X\| .
$$

In particular, if every element of $X$ has rank one, then

$$
\lambda_{M}(X)=r_{M}(X)+r_{M^{*}}(X)-|X| .
$$

The next lemma contains another useful equation whose proof is straightforward.

Lemma 29. Let $(X, Y)$ be a partition of the ground set of a polymatroid $M$. Suppose $z \in Y$. Then

$$
\sqcap(X,\{z\})+\sqcap_{M / z}(X, Y-z)=\sqcap(X,\{z\})+\lambda_{M / z}(X)=\lambda_{M}(X) .
$$

The next two lemmas are natural generalizations of matroid results that appear in [15].

Lemma 30. Let $(E, r)$ be a polymatroid and let $X$ and $Y$ be disjoint subsets of $E$. Then

$$
\lambda(X \cup Y)=\lambda(X)+\lambda(Y)-\sqcap(X, Y)-\Pi^{*}(X, Y) .
$$

Lemma 31. Let $A, B, C$, and $D$ be subsets of the ground set of a polymatroid. Then

(i) $\sqcap(A \cup B, C \cup D)+\sqcap(A, B)+\sqcap(C, D)=\sqcap(A \cup C, B \cup D)+\sqcap(A, C)+\sqcap(B, D)$; and

(ii) $\sqcap(A \cup B, C)+\sqcap(A, B)=\sqcap(A \cup C, B)+\sqcap(A, C)$.

Lemma 32. Let $M$ be a polymatroid and $(A, B, Z)$ be a partition of its ground set into possibly empty subsets. Then

$$
\lambda_{M / Z}(A)=\lambda_{M \backslash Z}(A)-\sqcap_{M}(A, Z)-\sqcap_{M}(B, Z)+\lambda_{M}(Z) .
$$

Proof. We have $B=A \cup Z$ and $A=B \cup Z$. Then

$$
\begin{aligned}
\lambda_{M / Z}(A) & =r_{M / Z}(A)+r_{M / Z}(B)-r(M / Z) \\
& =r(A \cup Z)-r(Z)+r(B \cup Z)-r(Z)-r(M)+r(Z) \\
& =r(B)+r(A)-r(M)-r(Z) \\
& =r(A)+r(B)-r(M \backslash Z)+r(M \backslash Z)-r(M)-r(Z) \\
& =\lambda_{M \backslash Z}(A)+r(M \backslash Z)-r(M)-r(Z) .
\end{aligned}
$$


The required result holds if and only if

$$
\sqcap_{M}(A, Z)+\sqcap_{M}(B, Z)-\lambda_{M}(Z)=r(M)+r(Z)-r(M \backslash Z) .
$$

Now

$$
\begin{aligned}
\sqcap(A, Z)+\sqcap(B, Z)-\lambda_{M}(Z)= & r(A)+r(Z)-r(A \cup Z)+r(B)+r(Z)-r(B \cup Z) \\
& -r(Z)-r(M \backslash Z)+r(M) \\
= & r(A)+r(Z)-r(B)+r(B)+r(Z)-r(A)-r(Z) \\
= & -r(M \backslash Z)+r(M) \\
= & -r(Z)-r(M \backslash Z),
\end{aligned}
$$

as required.

Corollary 33. Let $M$ be a polymatroid and $(A, B, Z)$ be a partition of its ground set into possibly empty subsets. Suppose $r(M \backslash Z)=r(M)$. Then

$$
\lambda_{M / Z}(A)=\lambda_{M \backslash Z}(A)-\sqcap_{M}(A, Z)-\sqcap_{M}(B, Z)+r(Z) .
$$

Proof. As $\lambda_{M}(Z)=r(Z)+r(M \backslash Z)-r(M)$ and $r(M \backslash Z)=r(M)$, the result is an immediate consequence of the last lemma.

Lemma 34. Let $M$ be a polymatroid and $(A, B, C)$ be a partition of its ground set into possibly empty subsets. Suppose $\lambda(A)=1=\lambda(C)$ and $\lambda(B)=2$. Then $\sqcap(A, B)=1$.

Proof. We have

$$
2=r(B)+r(A \cup C)-r(M)
$$

and

$$
\begin{aligned}
r(M) & =r(A \cup B)+r(C)-1 \\
& =r(A)+r(B)-\sqcap(A, B)+r(C)-1 .
\end{aligned}
$$

Thus

$$
2=r(B)+r(A \cup C)-r(A)-r(B)-r(C)+1+\sqcap(A, B)
$$

so $\sqcap(A, B)=1+\sqcap(A, C) \geqslant 1$. By Lemma $25, \sqcap(A, B) \leqslant \sqcap(A, B \cup C)=1$, so $\sqcap(A, B)=$ 1 .

Lemma 35. Let $M$ be a polymatroid and $(A, B, C)$ be a partition of its ground set into possibly empty subsets. Then $\Pi^{*}(A, B)=\lambda_{M / C}(A)$.

Proof. By making repeated use of Lemma 2, we have

$$
\Pi^{*}(A, B)=\sqcap_{M^{*}}(A, B)=\lambda_{M^{*} \backslash C}(A)=\lambda_{\left(M^{*} \backslash C\right)^{b}}(A)=\lambda_{(M / C)^{*}}(A)=\lambda_{M / C}(A) .
$$

The following is a consequence of a result of Oxley and Whittle [18, Lemma 3.1]. 
Lemma 36. Let $M$ be a 2-connected 2-polymatroid with $|E(M)| \geqslant 2$. If $e$ is a point of $M$, then $M \backslash e$ or $M / e$ is 2-connected.

Lemma 37. Let $M$ be a 3-connected 2-polymatroid having a and $\ell$ as distinct elements. Then $(E(M)-\{a, \ell\},\{\ell\})$ is not a 2-separation of $M / a$.

Proof. Assume the contrary. Then $\ell$ is a line in $M / a$, so $\sqcap(a, \ell)=0$. We have

$$
r_{M / a}(E(M)-\{a, \ell\})+r_{M / a}(\ell)=r(M / a)+1 .
$$

As $\sqcap(a, \ell)=0$, it follows that $(E(M)-\ell,\{\ell\})$ is a 2-separation of $M$, a contradiction.

The next result is another straightforward extension of a matroid result.

Lemma 38. Let $M$ be a 2-connected 2-polymatroid having e and $f$ as points. Then

(i) $\lambda_{M / f}(\{e\})=0$ if and only if $e$ and $f$ are parallel points; and

(ii) $\lambda_{M \backslash f}(\{e\})=0$ if and only if $e$ and $f$ form a series pair.

Proof. We prove (i) omitting the similar proof of (ii). If $e$ and $f$ are parallel points of $M$, then $\lambda_{M / f}(\{e\})=0$. Now assume that $\lambda_{M / f}(\{e\})=0$. Let $M^{\prime}$ be the natural matroid derived from $M$. Then $M^{\prime} / f$ has $\{e\}$ as a component. Hence $\{e, f\}$ is a series or parallel pair in $M^{\prime}$. But if $\{e, f\}$ is a series pair, then $M^{\prime} / f$ is 2-connected, a contradiction. We conclude that $\{e, f\}$ is a parallel pair of points in $M$, so (i) holds.

There is an attractive link between the connectivity of a 2-polymatroid $M$ and the connectivity of the natural matroid associated with $M$.

Lemma 39. Let $M$ be a 2-polymatroid with at least two elements and let $M^{\prime}$ be the natural matroid derived from $M$. Then

(i) $M$ is 2-connected if and only if $M^{\prime}$ is 2-connected; and

(ii) $M$ is 3-connected if and only if $M^{\prime}$ is 3-connected.

Proof. The result is immediate if $M$ is a matroid or has a loop, so we may assume that $M$ is loopless and has at least one line. Let $L$ be the set of lines of $M$ and let $M^{+}$be the matroid that is obtained from $M$ by freely adding two points on each line in $L$. Then $M^{\prime}=M^{+} \backslash L$.

Suppose that $M$ has a $k$-separation $(X, Y)$ for some $k$ in $\{1,2\}$. Replacing each line in each of $X$ and $Y$ by two points freely placed on the line gives sets $X^{\prime}$ and $Y^{\prime}$ that partition $E\left(M^{\prime}\right)$ such that $r\left(X^{\prime}\right)=r(X)$ and $r\left(Y^{\prime}\right)=r(Y)$. Hence $\left(X^{\prime}, Y^{\prime}\right)$ is a $k$-separation of $M^{\prime}$.

Now suppose that $M^{\prime}$ has a $k$-separation for some $k$ in $\{1,2\}$. Choose such a $k$ separation $\left(X^{\prime}, Y^{\prime}\right)$ to minimize the number $m$ of lines of $M$ that have exactly one of the corresponding points of $M^{\prime}$ in $X^{\prime}$. If $m=0$, then there is a $k$-separation of $M$ 
that corresponds naturally to $\left(X^{\prime}, Y^{\prime}\right)$. Thus we may assume that $M$ has a line $\ell$ whose corresponding points, $s_{\ell}$ and $t_{\ell}$, are in $X^{\prime}$ and $Y^{\prime}$, respectively. Now

$$
r\left(X^{\prime}\right)+r\left(Y^{\prime}\right)-r\left(M^{\prime}\right)=k-1 .
$$

Suppose $\left|E\left(M^{\prime}\right)\right|=3$. Then $M$ consists of a point and a line. For each $n$ in $\{2,3\}$, both $M$ and $M^{\prime}$ are $n$-connected if and only if the point lies on the line. Thus the result holds if $\left|E\left(M^{\prime}\right)\right|=3$. Now assume that $\left|E\left(M^{\prime}\right)\right|=4$. Then $M$ consists of either two lines, or a line and two points. Again the result is easily checked. Thus we may assume that $\left|E\left(M^{\prime}\right)\right| \geqslant 5$. We may also assume that $\left|X^{\prime}\right| \geqslant\left|Y^{\prime}\right|$. Then $\left|X^{\prime}\right| \geqslant 3$. Now $r\left(X^{\prime}-s_{\ell}\right)+r\left(Y^{\prime} \cup s_{\ell}\right)-r\left(M^{\prime}\right) \geqslant k$, otherwise the choice of $\left(X^{\prime}, Y^{\prime}\right)$ is contradicted. Thus $r\left(X^{\prime}-s_{\ell}\right)=r\left(X^{\prime}\right)$ and $r\left(Y^{\prime} \cup s_{\ell}\right)=r\left(Y^{\prime}\right)+1$. Hence, in $M^{+}$, as $s_{\ell}$ and $t_{\ell}$ are freely placed on $\ell$, we see that $r\left(\left(X^{\prime}-s_{\ell}\right) \cup \ell\right)=r\left(X^{\prime}-s_{\ell}\right)$, so

$$
r\left(X^{\prime}-s_{\ell}\right)=r\left(\left(X^{\prime}-s_{\ell}\right) \cup t_{\ell}\right)=r\left(X^{\prime} \cup t_{\ell}\right) .
$$

Hence $\left(X^{\prime} \cup t_{\ell}, Y^{\prime}-t_{\ell}\right)$ violates the choice of $\left(X^{\prime}, Y^{\prime}\right)$ unless either $k=1$ and $Y^{\prime}=$ $\left\{t_{\ell}\right\}$, or $k=2$ and $Y^{\prime}$ consists of two points. In the first case, $r\left(X^{\prime}\right)=r\left(M^{\prime}\right)$, so $r\left(X^{\prime}\right)+r\left(Y^{\prime}\right)-r\left(M^{\prime}\right)=1$, a contradiction to (10). In the second case, since one of the points in $Y^{\prime}$ is $t_{\ell}$, the points are not parallel so $r\left(Y^{\prime}\right)=2$ and $r\left(X^{\prime}\right)=r\left(M^{\prime}\right)-1$. Thus $r\left(X^{\prime} \cup t_{\ell}\right)=r\left(M^{\prime}\right)-1$ and $r\left(Y^{\prime}-t_{\ell}\right)=1$, a contradiction to (10).

The next result is a generalization of a lemma of Bixby [2] (see also [14, Lemma 8.7.3]) that is widely used when dealing with 3 -connected matroids.

Lemma 40. Let $M$ be a 3-connected 2-polymatroid and $z$ be a point of $M$. Then either

(i) $M / z$ is 2-connected having one side of every 2-separation being a pair of points of $M$ that are parallel in $M / z$; or

(ii) $M \backslash z$ is 2-connected having one side of every 2-separation being either a single line of $M$, or a pair of points of $M$ that form a series pair in $M \backslash z$.

Proof. If $z$ lies on a line in $M$, then $M \backslash z$ is 3-connected. Thus we may assume that $z$ does not lie on a line in $M$. Take the matroid $M^{\prime}$ that is naturally derived from $M$. Then, by Bixby's Lemma, either $M^{\prime} / z$ is 2-connected having one side of every 2-separation being a pair of parallel points of $M^{\prime}$, or $M^{\prime} \backslash z$ is 2-connected having one side of every 2-separation being a series pair of points of $M^{\prime}$. In the first case, if $\{a, b\}$ is a parallel pair of points of $M^{\prime} / z$, then $\{a, b, z\}$ is a circuit of $M^{\prime}$. Because the points added to $M$ to form $M^{\prime}$ are freely placed on lines, we cannot have a circuit containing just one of them. Since $z$ is not on a line of $M$, we deduce that $a$ and $b$ are points of $M$. We conclude that, in the first case, (i) holds.

Now suppose that $M^{\prime} \backslash z$ is not 3-connected and has $\{u, v\}$ as a series pair. Then either $u$ and $v$ are both matroid points of $M$, or $M$ has a line on which the points $u$ and $v$ are freely placed in the formation of $M^{\prime}$. We deduce that (ii) holds. 
We recall from [16] that, when $\{a, b, c\}$ is a set of three points in a 2-polymatroid $Q$, we call $\{a, b, c\}$ a triangle if every subset of $\{a, b, c\}$ of size at least two has rank two. If, instead, $r(E-\{a, b, c\})=r(Q)-1$ but $r(X)=r(Q)$ for all proper supersets $X$ of $E-\{a, b, c\}$, then we call $\{a, b, c\}$ a triad of $Q$. When $Q$ is 3-connected, $\{a, b, c\}$ is a triad of $Q$ if and only if $\{a, b, c\}$ is a triangle of $Q^{*}$. It is straightforward to check that a triangle and a triad of $Q$ cannot have exactly one common element. Just as for matroids, we call a sequence $x_{1}, x_{2}, \ldots, x_{k}$ of distinct points of a 2-polymatroid $Q$ a fan of length $k$ if $k \geqslant 3$ and the sets $\left\{x_{1}, x_{2}, x_{3}\right\},\left\{x_{2}, x_{3}, x_{4}\right\}, \ldots,\left\{x_{k-2}, x_{k-1}, x_{k}\right\}$ are alternately triangles and triads beginning with either a triangle or a triad.

The next two results were proved in [16, Lemmas 4.1 and 4.2]. The second can also be proved by applying Tutte's Triangle Lemma for matroids (see, for example, [14, Lemma 8.7.7]) to the matroid $M^{\prime}$ that is naturally derived from $M$ and using Lemma 39.

Lemma 41. Let $M$ be a 3-connected 2-polymatroid having a point $p$ such that neither $M \backslash p$ nor $M / p$ is 3 -connected. Then $M$ has points $s$ and $t$ such that $\{p, s, t\}$ is a triangle or a triad of $M$.

Lemma 42. Let $M$ be a 3-connected 2-polymatroid having at least four elements.

(i) Let $\{e, f, g\}$ be a triangle of $M$ such that neither $M \backslash e$ nor $M \backslash f$ is 3-connected. Then $M$ has a triad containing e and exactly one of $f$ and $g$.

(ii) Let $\{e, f, g\}$ be a triad of $M$ such that neither $M / e$ nor $M / f$ is 3-connected. Then $M$ has a triangle containing $e$ and exactly one of $f$ and $g$.

The following lemma will be helpful in proving our main result when fans arise in the argument.

Lemma 43. Let $M$ and $N$ be 3-connected 2-polymatroids where $|E(N)| \geqslant 4$ and $M$ is not a whirl or the cycle matroid of a wheel. Suppose $M$ has a fan $x_{1}, x_{2}, x_{3}, x_{4}$ where $\left\{x_{1}, x_{2}, x_{3}\right\}$ is a triangle and $M / x_{2}$ has a c-minor isomorphic to $N$. Then $M$ has a point $z$ such that either $M \backslash z$ or $M / z$ is 3-connected having a c-minor isomorphic to $N$, or both $M \backslash z$ and $M / z$ have c-minors isomorphic to $N$.

Proof. Assume that the lemma fails. Extend $x_{1}, x_{2}, x_{3}, x_{4}$ to a maximal fan $x_{1}, x_{2}, \ldots, x_{n}$. Since $M / x_{2}$ has a c-minor isomorphic to $N$ and has $x_{1}$ and $x_{3}$ as a parallel pair of points, it follows that each of $M / x_{2} \backslash x_{1}$ and $M / x_{2} \backslash x_{3}$ has a c-minor isomorphic to $N$. Thus each of $M \backslash x_{1}$ and $M \backslash x_{3}$ has a c-minor isomorphic to $N$. Hence $M / x_{4}$ has a c-minor isomorphic to $N$. A straightforward induction argument establishes that $M / x_{i}$ has a cminor isomorphic to $N$ for all even $i$, while $M \backslash x_{i}$ has a c-minor isomorphic to $N$ for all odd $i$. Then $M / x_{i}$ is not 3-connected when $i$ is even, while $M \backslash x_{i}$ is not 3 -connected when $i$ is odd.

Next we show that

43.1. $M$ has no triangle that contains more than one element $x_{i}$ with $i$ even; and $M$ has no triad that contains more than one element $x_{i}$ with $i$ odd. 
Suppose $M$ has a triangle that contains $x_{i}$ and $x_{j}$ where $i$ and $j$ are distinct even integers. Since $M / x_{i}$ has $x_{j}$ in a parallel pair of points, $M / x_{i} \backslash x_{j}$, and hence $M \backslash x_{j}$, has a c-minor isomorphic to $N$. As $M / x_{j}$ also has a c-minor isomorphic to $N$, we have a contradiction. Thus the first part of 43.1 holds. A similar argument proves the second part.

Suppose $n$ is odd. Then, since neither $M \backslash x_{n}$ nor $M \backslash x_{n-2}$ is 3-connected, by Lemma 42, $M$ has a triad $T^{*}$ containing $x_{n}$ and exactly one of $x_{n-1}$ and $x_{n-2}$. By 43.1, $T^{*}$ contains $x_{n-1}$. Then, by the maximality of the fan, the third element of $T^{*}$ lies in $\left\{x_{1}, x_{2}, \ldots, x_{n-2}\right\}$. But, as each of the points in the last set is in a triangle that is contained in that set, we obtain the contradiction that $M$ has a triangle having a single element in common with the triad $T^{*}$.

We may now assume that $n$ is even. As neither $M / x_{n}$ nor $M / x_{n-2}$ is 3 -connected, by Lemma $42, M$ has a triangle $T$ that contains $x_{n}$ and exactly one of $x_{n-1}$ and $x_{n-2}$. By 43.1, $x_{n-1} \in T$. The maximality of the fan again implies that the third element of $T$ is in $\left\{x_{1}, x_{2}, x_{3}, \ldots, x_{n-2}\right\}$. As every element of the last set, except $x_{1}$, is in a triad that is contained in the set, to avoid having $T$ meet such a triad in a single element, we must have that $T=\left\{x_{n}, x_{n-1}, x_{1}\right\}$. If $n=4$, then $M \mid\left\{x_{1}, x_{2}, x_{3}, x_{4}\right\} \cong U_{2,4}$ so $\left\{x_{2}, x_{3}, x_{4}\right\}$ is a triangle, a contradiction to 43.1. We deduce that $n>4$. Now neither $M \backslash x_{1}$ nor $M \backslash x_{n-1}$ is 3-connected. Thus, by Lemma $42, M$ has a triad $T_{2}^{*}$ containing $x_{1}$ and exactly one of $x_{n}$ and $x_{n-1}$. By 43.1, $x_{n} \in T_{2}^{*}$. The triangles $\left\{x_{1}, x_{2}, x_{3}\right\}$ and $\left\{x_{3}, x_{4}, x_{5}\right\}$ imply that $x_{2} \in T_{2}^{*}$. Let $X=\left\{x_{1}, x_{2}, \ldots, x_{n}\right\}$. Then, using the triangles we know, including $\left\{x_{n}, x_{n-1}, x_{1}\right\}$, we deduce that $r(X) \leqslant \frac{n}{2}$. Similarly, the triads in $M$, which are triangles in $M^{*}$, imply that $r^{*}(X) \leqslant \frac{n}{2}$. Thus, by Lemma $28, \lambda(X)=0$. Hence $X=E(M)$. As every element of $M$ is a point, $M$ is a matroid. Since every point of $M$ is in both a triangle and a triad, by Tutte's Wheels-and-Whirls-Theorem [21], we obtain the contradiction that $M$ is a whirl or the cycle matroid of a wheel.

\section{Parallel connection and 2-sum}

In this section, we follow Matúš [11] and Hall [5] in defining the parallel connection and 2-sum of polymatroids. For a positive integer $k$, let $M_{1}$ and $M_{2}$ be $k$-polymatroids $\left(E_{1}, r_{1}\right)$ and $\left(E_{2}, r_{2}\right)$. Suppose first that $E_{1} \cap E_{2}=\emptyset$. The direct sum $M_{1} \oplus M_{2}$ of $M_{1}$ and $M_{2}$ is the $k$-polymatroid $\left(E_{1} \cup E_{2}, r\right)$ where, for all subsets $A$ of $E_{1} \cup E_{2}$, we have $r(A)=r\left(A \cap E_{1}\right)+r\left(A \cap E_{2}\right)$. The following result is easily checked.

Lemma 44. For k-polymatroids $M_{1}$ and $M_{2}$ on disjoint sets,

$$
\left(M_{1} \oplus M_{2}\right)^{*}=M_{1}^{*} \oplus M_{2}^{*} .
$$

Clearly a 2-polymatroid is 2-connected if and only if it cannot be written as the direct sum of two non-empty 2-polymatroids. Now suppose that $E_{1} \cap E_{2}=\{p\}$ and $r_{1}(\{p\})=r_{2}(\{p\})$. Let $P\left(M_{1}, M_{2}\right)$ be $\left(E_{1} \cup E_{2}, r\right)$ where $r$ is defined for all subsets $A$ of $E_{1} \cup E_{2}$ by

$$
r(A)=\min \left\{r_{1}\left(A \cap E_{1}\right)+r_{2}\left(A \cap E_{2}\right), r_{1}\left(\left(A \cap E_{1}\right) \cup p\right)+r_{2}\left(\left(A \cap E_{2}\right) \cup p\right)-r_{1}(\{p\})\right\} .
$$


As Hall notes, it is routine to check that $P\left(M_{1}, M_{2}\right)$ is a $k$-polymatroid. We call it the parallel connection of $M_{1}$ and $M_{2}$ with respect to the basepoint $p$. When $M_{1}$ and $M_{2}$ are both matroids, this definition coincides with the usual definition of the parallel connection of matroids. Hall extends the definition of parallel connection to deal with the case when $r_{1}(\{p\}) \neq r_{2}(\{p\})$ but we shall not do that here.

Now suppose that $M_{1}$ and $M_{2}$ are 2-polymatroids having at least two elements, that $E\left(M_{1}\right) \cap E\left(M_{2}\right)=\{p\}$, that neither $\lambda_{M_{1}}(\{p\})$ nor $\lambda_{M_{2}}(\{p\})$ is 0 , and that $r_{1}(\{p\})=$ $r_{2}(\{p\})=1$. We define the 2-sum, $M_{1} \oplus_{2} M_{2}$, of $M_{1}$ and $M_{2}$ to be $P\left(M_{1}, M_{2}\right) \backslash p$. We remark that this extends Hall's definition since, to ensure that $M_{1} \oplus_{2} M_{2}$ has more elements than each of $M_{1}$ and $M_{2}$, he requires that they each have at least three elements. He imposes the same requirement in his Proposition 3.6. The next result is that result with this restriction omitted. Hall's proof [5] remains valid.

Proposition 45. Let $M$ be a 2-polymatroid $(E, r)$ having a partition $\left(X_{1}, X_{2}\right)$ such that $r\left(X_{1}\right)+r\left(X_{2}\right)=r(E)+1$. Then there are 2-polymatroids $M_{1}$ and $M_{2}$ with ground sets $X_{1} \cup p$ and $X_{2} \cup p$, where $p$ is a new element not in $E$, such that $M=P\left(M_{1}, M_{2}\right) \backslash p$. In particular, for all $A \subseteq X_{1} \cup p$,

$$
r_{1}(A)= \begin{cases}r(A), & \text { if } p \notin A ; \\ r\left((A-p) \cup X_{2}\right)-r\left(X_{2}\right)+1, & \text { if } p \in A .\end{cases}
$$

Lemma 46. Let $(X, Y)$ be a partition of the ground set of a 2-polymatroid $M$ such that $\lambda(X)=1$. Then, for some element $p$ not in $E(M)$, there are 2-polymatroids $M_{X}$ and $M_{Y}$ on $X \cup p$ and $Y \cup p$, respectively, such that $M=M_{X} \oplus_{2} M_{Y}$. Moreover, for $y \in Y$,

(i) $\lambda_{M_{Y} \backslash y}(\{p\})=\sqcap(X, Y-y)$;

(ii) $\lambda_{M_{Y} / y}(\{p\})+\sqcap(X,\{y\})=\lambda(X)=1$;

(iii) if $\sqcap(X, Y-y)=1$, then $M \backslash y=M_{X} \oplus_{2}\left(M_{Y} \backslash y\right)$;

(iv) if $\sqcap(X,\{y\})=0$, then $M / y=M_{X} \oplus_{2}\left(M_{Y} / y\right)$; and

(v) if $r(\{y\}) \leqslant 1$, then

$$
M \downarrow y= \begin{cases}\left(M_{X} / p\right) \oplus\left(M_{Y} \backslash y / p\right), & \text { if } \sqcap(X,\{y\})=1 ; \\ M_{X} \oplus_{2}\left(M_{Y} \downarrow y\right), & \text { if } \sqcap(X,\{y\})=0 .\end{cases}
$$

(vi) if $y$ is a line, then

$$
M \downarrow y= \begin{cases}\left(M_{X} \backslash p\right) \oplus\left(M_{Y} \backslash y \backslash p\right), & \text { if } r(Y)=r(Y-y)+2 ; \\ M_{X} \oplus_{2}\left(M_{Y} \downarrow y\right) & \text { if } r(Y) \leqslant r(Y-y)+1 .\end{cases}
$$

In particular, $M \downarrow y=M_{X} \oplus_{2}\left(M_{Y} \downarrow y\right)$ when $\Pi_{M \downarrow y}(X, Y-y)=1$. 
Proof. The existence of $M_{X}$ and $M_{Y}$ such that $M=P\left(M_{X}, M_{Y}\right) \backslash p$ is an immediate consequence of Proposition 45. To see that $P\left(M_{X}, M_{Y}\right) \backslash p=M_{X} \oplus_{2} M_{Y}$, one needs only to check that $r_{M_{X}}(\{p\})=1=r_{M_{Y}}(\{p\})$ and $\lambda_{M_{X}}(\{p\})=1=\lambda_{M_{Y}}(\{p\})$.

The proof of (i) follows by a straightforward application of the rank formula in Proposition 45. We omit the details. To see that (ii) holds, note that

$$
\begin{aligned}
\lambda_{M_{Y} / y}(\{p\}) & =r_{M_{Y}}(\{p, y\})-r(\{y\})+r_{M_{Y}}(Y)-r_{M_{Y}}(Y \cup p) \\
& =r(y \cup X)-r(X)+1-r(\{y\})+r(Y)-r(X \cup Y)+r(X)-1 \\
& =r(y \cup X)-r(\{y\})+r(Y)-r(X \cup Y) \\
& =r(X)-\sqcap(X,\{y\})+r(Y)-r(X \cup Y) \\
& =\lambda_{M}(X)-\sqcap(X,\{y\}) .
\end{aligned}
$$

By Hall [5, Proposition 3.1], $M \backslash y=P\left(M_{X}, M_{Y} \backslash y\right) \backslash p$. If $\sqcap(X, Y-y)=1$, then, by (i), $\lambda_{M_{Y} \backslash y}(\{p\})=1$. Hence, by Hall [5, Proposition 3.1], $M \backslash y=M_{X} \oplus_{2}\left(M_{Y} \backslash y\right)$; that is, (iii) holds.

To prove (iv), assume that $\sqcap(X,\{y\})=0$. We could again follow Hall [5, Proposition 3.1] to get that $M / y=P\left(M_{X}, M_{Y} / y\right) \backslash p$. But since he omits a full proof of this fact, we include it for completeness.

By Proposition $45, M / y=P\left(M_{1}, M_{2}\right) \backslash p$ for some $M_{1}$ and $M_{2}$. For $A \subseteq X \cup p$,

$$
\begin{aligned}
r_{M_{1}}(A) & = \begin{cases}r_{M / y}(A), & \text { if } p \notin A ; \\
r_{M / y}((A-p) \cup(Y-y))-r_{M / y}(Y-y)+1, & \text { if } p \in A ;\end{cases} \\
& = \begin{cases}r(A \cup y)-r(\{y\}), & \text { if } p \notin A ; \\
r((A-p) \cup Y)-r(Y)+1, & \text { if } p \in A ;\end{cases} \\
& =r_{M_{X}}(A) .
\end{aligned}
$$

Thus $M_{1}=M_{X}$.

Now, for $A \subseteq(Y-y) \cup p$,

$$
\begin{aligned}
r_{M_{2}}(A) & = \begin{cases}r_{M / y}(A), & \text { if } p \notin A ; \\
r_{M / y}((A-p) \cup X)-r_{M / y}(X)+1, & \text { if } p \in A ;\end{cases} \\
& = \begin{cases}r(A \cup y)-r(\{y\}), & \text { if } p \notin A ; \\
r((A-p) \cup X \cup y)-r(\{y\})-r(X \cup y)+r(\{y\})+1, & \text { if } p \in A ;\end{cases} \\
= & \begin{cases}r(A \cup y)-r(\{y\}), & \text { if } p \notin A ; \\
r((A-p) \cup X \cup y)-r(\{y\})-r(X)+1, & \text { if } p \in A .\end{cases}
\end{aligned}
$$

But

$$
\begin{aligned}
r_{M_{Y} / y}(A) & =r_{M_{Y}}(A \cup y)-r_{M_{Y}}(\{y\}) \\
& = \begin{cases}r(A \cup y)-r(\{y\}), & \text { if } p \notin A ; \\
r((A-p) \cup X \cup y)-r(\{y\})-r(X)+1, & \text { if } p \in A ;\end{cases} \\
& =r_{M_{2}}(A) .
\end{aligned}
$$


Thus $M_{2}=M_{Y} / y$, so $M / y=P\left(M_{X}, M_{Y} / y\right) \backslash p$. As $\sqcap(X,\{y\})=0$, we see, by (ii), that $\lambda_{M_{Y} / y}(\{p\})=1$. Hence $M / y=M_{X} \oplus_{2}\left(M_{Y} / y\right)$; that is, (iv) holds.

For (v), since $r(\{y\}) \leqslant 1$, we have $M \downarrow y=M / y$. If $\sqcap(X,\{y\})=1$, then $y$ is parallel to $p$ in $M_{Y}$, so, by [5, Proposition 3.1], $M \downarrow y=\left(M_{X} / p\right) \oplus\left(M_{Y} / p\right)$. If $\sqcap(X,\{y\})=0$, then, as $M_{Y} \downarrow y=M_{Y} / y$, it follows by (iv) that

$$
M \downarrow y=M / y=M_{X} \oplus_{2}\left(M_{Y} / y\right)=M_{X} \oplus_{2}\left(M_{Y} \downarrow y\right) .
$$

To prove (vi), suppose first that $r(Y)=r(Y-y)+2$. We have

$$
r_{M_{Y}}(\{y, p\})=r(y \cup X)-r(X)+1=3-\sqcap(X,\{y\}) .
$$

Assume $\sqcap(X,\{y\})=0$. Then $M_{Y}$ is the 2-sum, with basepoint $q$, say, of two 2polymatroids, one of which has ground set $\{q, y, p\}$ and consists of two points and the line $y$ freely placed in the plane. Clearly, $M \downarrow y=\left(M_{X} \backslash p\right) \oplus\left(M_{Y} \backslash y \backslash p\right)$. Now assume that $\sqcap(X,\{y\})=1$. Then $M_{Y}$ is the direct sum of two 2-polymatroids, one of which has rank 2 and consists of the line $y$ with the point $p$ on it. Once again, we see that $M \downarrow y=\left(M_{X} \backslash p\right) \oplus\left(M_{Y} \backslash y \backslash p\right)$.

We may now assume that $r(Y) \leqslant r(Y-y)+1$. Hence $r_{M \downarrow y}(Y-y)=r(Y)-1$. Clearly $r(X \cup y)>r(X)$. Thus

$$
\sqcap_{M \downarrow y}(X, Y-y)=1 .
$$

By Proposition 45, $M \downarrow y=P\left(M_{1}, M_{2}\right) \backslash p$ for some 2-polymatroids $M_{1}$ and $M_{2}$ with ground sets $X \cup p$ and $(Y-y) \cup p$, respectively. We shall show that $M_{1}=M_{X}$ and $M_{2}=M_{Y} \downarrow y$.

First observe that, for $A \subseteq X$, we have

$$
r_{M_{1}}(A)= \begin{cases}r_{M \downarrow y}(A), & \text { if } p \notin A ; \\ r_{M \downarrow y}((A-p) \cup(Y-y))-r_{M \downarrow y}(Y-y)+1, & \text { if } p \in A .\end{cases}
$$

Since $r(X \cup y)>r(X)$, we see that if $p \notin A$, then $r_{M_{1}}(A)=r_{M \downarrow y}(A)=r_{M}(A)=r_{M_{X}}(A)$.

Now suppose $p \in A$. Assume $r((A-p) \cup(Y-y))=r((A-p) \cup Y)$. Then

$$
r_{M \downarrow y}((A-p) \cup(Y-y))=r((A-p) \cup(Y-y))-1=r((A-p) \cup Y)-1 .
$$

Moreover, $r_{M \downarrow y}(Y-y)=r(Y)-1$. Hence

$$
r_{M_{1}}(A)=r_{M}((A-p) \cup Y)-r_{M}(Y)+1=r_{M_{X}}(A) .
$$

To show that $M_{1}=M_{X}$, it remains to consider when $p \in A$ and $r((A-p) \cup(Y-y))<$ $r((A-p) \cup Y)$. Then, as $r(Y-y) \geqslant r(Y)-1$, we deduce that $r((A-p) \cup(Y-y))=$ $r((A-p) \cup Y)-1$, so $r(Y-y)=r(Y)-1$. Thus we have

$$
\begin{aligned}
r_{M_{1}}(A) & =r_{M \downarrow y}((A-p) \cup(Y-y))-r_{M \downarrow y}(Y-y)+1 \\
& =r((A-p) \cup(Y-y))-r(Y-y)+1 \\
& =r((A-p) \cup Y)-1-r(Y)+1+1 \\
& =r_{M_{X}}(A) .
\end{aligned}
$$


We conclude that $M_{1}=M_{X}$.

To show that $M_{2}=M_{Y} \downarrow y$, suppose that $A \subseteq(Y-y) \cup p$. Now

$$
r_{M_{2}}(A)= \begin{cases}r_{M \downarrow y}(A), & \text { if } p \notin A ; \\ r_{M \downarrow y}((A-p) \cup X)-r_{M \downarrow y}(X)+1, & \text { if } p \in A .\end{cases}
$$

Suppose $p \notin A$. Then

$$
\begin{aligned}
r_{M_{2}}(A) & = \begin{cases}r(A), & \text { if } r(A \cup y)>r(A) ; \\
r(A)-1, & \text { otherwise; }\end{cases} \\
& =r_{M_{Y} \downarrow y}(A) .
\end{aligned}
$$

Now assume that $p \in A$. Then $r_{M \downarrow y}(X)=r(X)$. Thus

$$
r_{M_{2}}(A)= \begin{cases}r((A-p) \cup X)-r(X)+1, & \text { if } r((A-p) \cup X \cup y)>r((A-p) \cup X) ; \\ r((A-p) \cup X)-1-r(X)+1, & \text { otherwise. }\end{cases}
$$

Moreover,

$$
r_{M_{Y} \downarrow y}(A)= \begin{cases}r_{M_{Y}}(A), & \text { if } r_{M_{Y}}(A \cup y)>r_{M_{Y}}(A) ; \\ r_{M_{Y}}(A)-1, & \text { otherwise. }\end{cases}
$$

Now $r_{M_{Y}}(A)=r((A-p) \cup X)-r(X)+1$. Thus

$$
\begin{gathered}
r_{M_{Y}}(A \cup y)-r_{M_{Y}}(A)=r((A-p) \cup y \cup X)-r(X)+1-r((A-p) \cup X) \\
+r(X)-1 \\
=r((A-p) \cup y \cup X)-r((A-p) \cup X) .
\end{gathered}
$$

We conclude that, when $p \in A$, we have $r_{M_{Y} \downarrow y}(A)=r_{M_{2}}(A)$. Thus $M_{Y} \downarrow y=M_{2}$. Hence $M \downarrow y=P\left(M_{X}, M_{Y} \downarrow y\right) \backslash p$. Using (11), it is straightforward to show that $\lambda_{M_{Y} \downarrow y}(\{p\})=1$. It follows that $M \downarrow y=M_{X} \oplus_{2}\left(M_{Y} \downarrow y\right)$.

The following was shown by Hall [5, Corollary 3.5].

Proposition 47. Let $M_{1}$ and $M_{2}$ be 2-polymatroids $\left(E_{1}, r_{1}\right)$ and $\left(E_{2}, r_{2}\right)$ where $E_{1} \cap E_{2}=$ $\{p\}$ and $r_{1}(\{p\})=r_{2}(\{p\})=1$ and each of $M_{1}$ and $M_{2}$ has at least two elements. Then the following are equivalent.

(i) $M_{1}$ and $M_{2}$ are both 2-connected;

(ii) $M_{1} \oplus_{2} M_{2}$ is 2-connected;

(iii) $P\left(M_{1}, M_{2}\right)$ is 2-connected.

One situation that will often occur will be when we have a certain 3-connected 2polymatroid $N$ arising as a c-minor of a 2-polymatroid $M$ that has a 2-separation. Recall that a special $N$-minor of $M$ is a c-minor of $M$ that either equals $N$ or differs from $N$ by having a single point relabelled. 
Lemma 48. Let $M$ be a 2-polymatroid that can be written as the 2-sum $M_{X} \oplus_{2} M_{Y}$ of 2-polymatroids $M_{X}$ and $M_{Y}$ with ground sets $X \cup p$ and $Y \cup p$, respectively. Let $N$ be a 3-connected 2-polymatroid with $|E(N)| \geqslant 4$ and $E(N) \subseteq E(M)$. If $M_{X}$ has a special $N$-minor, then $M$ has a special $N$-minor.

Proof. Since $M_{X} \backslash p=M \backslash Y$ and $M_{X} / p=M / Y$, we may assume that the special $N$-minor of $M_{X}$ uses $p$. Hence every other element of the special $N$-minor of $M_{X}$ is in $E(N)$. For $y$ in $Y$, we will denote by $M_{X}(y)$ the 2-polymatroid that is obtained from $M_{X}$ by relabelling $p$ by $y$. We argue by induction on $|Y|$. Take $y$ in $Y$.

Suppose $|Y|=1$. If $y$ is a point, then the result is immediate since $M=M_{X}(y)$. If $y$ is a line, then compactifying this line gives $M_{X}(y)$ and again the result holds.

Now suppose that $|Y|>1$. Suppose $\sqcap(\{y\}, X)=1$. Then $M \mid(X \cup y)=M_{X}(y)$ if $y$ is a point. If $y$ is a line, then compactifying $y$ in $M \mid(X \cup y)$ gives $M_{X}(y)$. In each case, the result holds. We may now assume that $\sqcap(\{y\}, X)=0$. Then, by Lemma 46(iv), $M / y=M_{X} \oplus_{2}\left(M_{Y} / y\right)$, so the result follows by induction.

Lemma 49. Let $M$ be a 2-polymatroid that can be written as the 2-sum $M_{X} \oplus_{2} M_{Y}$ of 2-polymatroids $M_{X}$ and $M_{Y}$ with ground sets $X \cup p$ and $Y \cup p$, respectively. Let $N$ be a 3-connected 2-polymatroid with $|E(N)| \geqslant 4$ such that $N$ is a c-minor of $M$. If $|E(N) \cap X| \geqslant|E(N)|-1$, then $M_{X}$ has a special $N$-minor that uses $E(N) \cap X$.

Proof. As $N$ is a c-minor of $M$, it follows by Corollary 8 that $N$ can be obtained from $M$ by a sequence of deletions and contractions followed by one compactification at the end. Let $N_{1}$ be the 2-polymatroid that is obtained prior to the last compactification. We know that we can shuffle these deletions and contractions at will. In producing $N_{1}$ from $M$, let $C_{Y}$ and $D_{Y}$ be the sets of elements of $Y$ that are contracted and deleted, respectively.

Suppose $\sqcap\left(X, C_{Y}\right)=1$. Now $M=P\left(M_{X}, M_{Y}\right) \backslash p$. Consider $P\left(M_{X}, M_{Y}\right) / C_{Y} \backslash D_{Y}$. This has $p$ as a loop, so $P\left(M_{X}, M_{Y}\right) \backslash p / C_{Y} \backslash D_{Y}=P\left(M_{X}, M_{Y}\right) / p / C_{Y} \backslash D_{Y}$. Because $P\left(M_{X}, M_{Y}\right) / p=\left(M_{X} / p\right) \oplus\left(M_{Y} / p\right)$, we deduce that $Y=D_{Y} \cup C_{Y}$, so $N_{1}$ is a c-minor of $M_{X} / p$. As we can perform a compactification whenever we want, $N$ is a c-minor of $\left(M_{X} / p\right)^{b}$ and hence of $M_{X}$.

We may now assume that $\sqcap\left(X, C_{Y}\right)=0$. Suppose $Y \cap E(N)=\emptyset$. Then $M \backslash D_{Y} / C_{Y}=$ $M \backslash Y=M_{X} \backslash p$. Hence $N_{1}$ is a c-minor of $M_{X}$, so $N$ is a c-minor of $\left(M_{X}\right)^{b}$. It remains to consider the case when $Y \cap E(N)$ consists of a single element, $y$. In $M / C_{Y} \backslash D_{Y}$, we must have $\sqcap(X,\{y\})=1$, otherwise $\sqcap(X,\{y\})=0$ and $\{y\}$ is 1-separating in $N_{1}$ and hence in $N$, a contradiction. We deduce that, in $M_{Y} / C_{Y} \backslash D_{Y}$, the element $y$ is either a point parallel to the basepoint $p$ or a line through $p$. In the latter case, $\left(M / C_{Y} \backslash D_{Y}\right)^{b}$ is $\left(M_{X}(y)\right)^{b}$ where $M_{X}(y)$ is obtained from $M_{X}$ by relabelling $p$ by $y$. In both cases, $\left(M_{X}(y)\right)^{b}$ has $N$ as a c-minor so $\left(M_{X}\right)^{b}$ and hence $M_{X}$ has a special $N$-minor.

Lemma 50. Let $p$ be a point in a 2-polymatroid $P$ having ground set $E$. If $\sqcap(p, E-p)=1$, then $P$ has as a minor a 2-element 2-connected 2-polymatroid using $p$.

Proof. We argue by induction on $|E-p|$. the result is certainly true if $|E-p|=1$. Assume it true for $|E-p|<n$ and let $|E-p|=n$. If $E-p$ contains an element $z$ 
such that $\sqcap(p, z)=1$, then the result is immediate. Thus $E-p$ contains an element $z$ such that $\sqcap(p, z)=0$. Then $\Pi_{P / z}(p, E-\{p, z\})=r(p)+r(E-p)-r(P)=1$. Thus, by the induction assumption, $P / z$ and hence $P$ has, as a minor, a 2-element 2-connected 2-polymatroid using $p$.

Lemma 51. Let $(X, Y)$ be an exact 2-separation of a 2-polymatroid $M$ and let $N$ be a 3-connected 2-polymatroid that is a c-minor of $M$. Suppose that $|E(N)-X| \leqslant 1$ and $y \in Y$.

(i) If $\sqcap_{M \backslash y}(X, Y-y)=1$, then $M \backslash y$ has a special $N$-minor.

(ii) If $\sqcap_{M / y}(X, Y-y)=1$, then $M / y$ has a special $N$-minor.

(iii) If $\sqcap_{M \downarrow y}(X, Y-y)=1$, then $M \downarrow y$ has a special $N$-minor.

Proof. By Lemma 46, $M=M_{X} \oplus_{2} M_{Y}$ where $M_{X}$ and $M_{Y}$ have ground sets $X \cup p$ and $Y \cup p$, respectively. By Lemma $49, M_{X}$ has a special $N$-minor using $E(N) \cap X$. Suppose $\sqcap_{M \backslash y}(X, Y-y)=1$. Then $\sqcap_{M_{Y}}(\{p\}, Y-y)=1$. Thus, by Lemma 50, $M_{Y} \backslash y$ has as a minor a 2-polymatroid with ground set $\{p, z\}$ for some $z$ in $Y-y$ where either $p$ and $z$ are parallel points, or $z$ is a line and $p$ is a point on this line. It follows that $(M \backslash y)^{b}$ has as a c-minor the 2-polymatroid that is obtained from $\left(M_{X}\right)^{b}$ by relabelling $p$ by $z$. Hence $M \backslash y$ has a special $N$-minor and (i) holds.

Now suppose that $\sqcap_{M / y}(X, Y-y)=1$. Then, by Lemma 29, $\sqcap(X,\{y\})=0$. Thus, by Lemma 46 (iv), $M / y=M_{X} \oplus_{2}\left(M_{Y} / y\right)$. Moreover, $\sqcap_{M_{Y} / y}(\{p\}, Y-y)=1$. Then, by replacing $M_{Y} \backslash y$ by $M_{Y} / y$ in the argument in the previous paragraph, we deduce that (ii) holds.

Finally, suppose that $\sqcap_{M \downarrow y}(X, Y-y)=1$. Assume first that $r(\{y\}) \leqslant 1$. Then $M \downarrow y=M / y$, so $\sqcap_{M / y}(X, Y-y)=1$, and the result follows by (ii). Now let $y$ be a line of $M$. Then, by Lemma 46(vi), $M \downarrow y=M_{X} \oplus_{2}\left(M_{Y} \downarrow y\right)$. Again, by replacing $M_{Y} \backslash y$ by $M_{Y} \downarrow y$ in the argument in the first paragraph, we get that (iii) holds.

Lemma 52. Let $Q$ be a 2-polymatroid having $k$ and $\ell$ as distinct elements and suppose that $\ell$ is a 2 -separating line. Then

$$
Q \downarrow \underline{\ell} \downarrow k=Q \downarrow k \downarrow \underline{\ell} .
$$

Proof. The result is easily checked if $\lambda(\ell)=0$, so assume that $\lambda(\ell)=1$. Then, by Lemma 46, $Q=P\left(Q_{1}, Q_{2}\right) \backslash p$ for some 2-polymatroids $Q_{1}$ and $Q_{2}$ with ground sets $(E(Q)-\ell) \cup p$ and $\{\ell, p\}$ where $Q_{2}$ consists of the line $\ell$ with the point freely placed on it. Moreover, either

(i) $k$ is a point that is parallel to $p$ in $Q_{1}$; or

(ii) $Q \downarrow k=P\left(Q_{1} \downarrow k, Q_{2}\right) \backslash p$. 
Consider the first case. Then $Q \downarrow k=Q / k$ and $Q \downarrow k \downarrow \ell$ can be obtained from $Q_{1} / p$ by adjoining $\ell$ as a loop. On the other hand, $Q \downarrow \ell$ can be obtained from $Q_{1}$ by relabelling $p$ as $\ell$. Thus $Q \downarrow \ell \downarrow k$, which equals $Q \downarrow \ell / k$, can be obtained from $Q_{1} / p$ by adjoining $\ell$ as a loop. Hence the result holds in case (i).

Now suppose (ii) holds. Then $Q \downarrow k \downarrow \ell$ can be obtained from $Q_{1} \downarrow k$ by relabelling $p$ as $\ell$. On the other hand, $Q \downarrow \ell \ell$ can be obtained from $Q_{1}$ by relabelling $p$ as $\ell$. Hence $Q \downarrow \ell \downarrow k$ can be obtained from $Q_{1} \downarrow k$ by relabelling $p$ as $\ell$. Thus the lemma holds.

We end this section with three lemmas concerning 2-element prickly 3-separators.

Lemma 53. Let $P$ be a compact 2-polymatroid $(E, r)$ having $\{j, k\}$ as a prickly 3separator. Suppose $y \in E-\{j, k\}$. If $\{j, k\}$ is not a prickly 3 -separator of $P / y$, then

(i) $r(\{j, k, y\})=3$ and $P / y$ has $\{j, k\}$ as a 1-separating set; or

(ii) $P \downarrow k / y=P / y \rrbracket k$; or

(iii) $P \downarrow k / y=P / y / k$; or

(iv) $P \downarrow k / y$ can be obtained from $P / y \rrbracket j$ by relabelling $k$ as $j$.

Proof. Suppose first that $r_{P / y}(\{j, k\})=1$. Then $y$ is a line of $P$ that is in the closure of $\{j, k\}$. Thus $\lambda_{P / y}(\{j, k\})=0$ and (i) holds.

Next assume that $r_{P / y}(\{j, k\})=2$. Then $\lambda_{P / y}(\{j, k\})=1$. Thus $P / y$ can be written as the 2-sum, with basepoint $p$ of two polymatroids, one of which, $P_{1}$, has ground set $\{j, k, p\}$ and has rank 2. As $P$ is compact, so is $P / y$. Hence neither $j$ nor $k$ is parallel to $p$. There are four choices for $P_{1}$ :

(a) $j$ and $k$ are parallel lines and $p$ is a point lying on them both;

(b) $P_{1}$ is isomorphic to the matroid $U_{2,3}$;

(c) $P_{1}$ has $k$ as a line and has $j$ and $p$ as distinct points on this line; or

(d) $P_{1}$ has $j$ as a line and has $k$ and $p$ as distinct points on this line;

By Lemma 18, $P \downarrow k / y=P / y \downarrow k$. If $P_{1}$ is one of the 2-polymatroids in (b) or (d), then, as $k$ is a point of $P / y$, it follows that $P / y \downarrow k=P / y / k$, so (iii) holds. Next suppose that $P_{1}$ is the 2-polymatroid in (a). Then, as $P / y$ is compact, it follows that $P / y \downarrow k=P / y \rrbracket k$, so (ii) holds. Finally, suppose that $P_{1}$ is the 2-polymatroid in (c). Then $P \downarrow j / y=P / y \downarrow j=P / y \| j$. By Lemma 16, $P \downarrow j$ can be obtained from $P \downarrow k$ by relabelling $j$ as $k$. Thus $P \downarrow j / y$ can be obtained from $P / y \| j$ by relabelling $k$ as $j$, that is, (iv) holds.

We may now assume that $r_{P / y}(\{j, k\})=3$. Then $\sqcap(\{y\},\{j, k\})=0$ and one easily checks that $\{j, k\}$ is a prickly 3 -separator of $P / y$, a contradiction.

Lemma 54. Let $\{j, k\}$ be a prickly 3-separator in a 3-connected 2-polymatroid $M$. Then $M \downarrow j$ and $M \downarrow k$ are 3-connected. 
Proof. It suffices to show that $M \downarrow j$ is 3-connected. We form $M \downarrow j$ by freely adding a point $j^{\prime}$ to $j$, deleting $j$, and contracting $j^{\prime}$. As $M$ is 3 -connected, so is the 2-polymatroid $M^{\prime}$ we get by adding $j^{\prime}$. Now $M \downarrow j=M^{\prime} \backslash j / j^{\prime}$. Assume this 2-polymatroid is not 3 -connected, letting $(U, V)$ be an $m$-separation of it for some $m$ in $\{1,2\}$. Then

$$
r_{M^{\prime} / j^{\prime}}(U)+r_{M^{\prime} / j^{\prime}}(V)=r\left(M^{\prime} / j^{\prime}\right)+m-1 .
$$

Thus

$$
r_{M^{\prime}}\left(U \cup j^{\prime}\right)+r_{M^{\prime}}\left(V \cup j^{\prime}\right)=r\left(M^{\prime}\right)+m .
$$

Without loss of generality, $k \in V$. Then $r_{M^{\prime}}\left(V \cup j^{\prime}\right)=r_{M}(V \cup j)$ and $r_{M^{\prime}}\left(U \cup j^{\prime}\right)=$ $r_{M}(U)+1$. Therefore

$$
r_{M}(U)+r_{M}(V \cup j)=r(M)+m-1 .
$$

As $M$ is 3 -connected, we deduce that $m=2$. Then $\max \left\{|U|, r_{M^{\prime} / j^{\prime}}(U)\right\} \geqslant 2$. Hence $(U, V \cup j)$ is a 2-separation of $M$, a contradiction.

Lemma 55. Let $\{j, k\}$ be a prickly 3-separator in a 2-polymatroid $P$. Then

(i) $P \downarrow k \backslash j=P \backslash k, j$; and

(ii) $P \downarrow k / j=P / k, j$.

Proof. Suppose $X \subseteq E(P)-\{j, k\}$. Then $r_{P \downarrow k}(X)=r_{P}(X)$ as $r(X \cup k)>r(X)$. Thus (i) holds.

To see (ii), observe that $r_{P \downarrow k / j}(X)=r_{P \downarrow k}(X \cup j)-r(\{j\})$ since $r(\{j, k\})>r(\{j\})$. Now, by Lemma 14, $r_{P \downarrow k}(X \cup j)=r(X \cup j \cup k)-1$. Thus $r_{P \downarrow k / j}(X)=r(X \cup j \cup k)-3=r_{P / k, j}(X)$, so (ii) holds.

\section{The strategy of the proof}

The proof of Theorem 4 is long and will occupy the rest of the paper. In this section, we outline the steps in the proof. We shall assume that the theorem fails for $M$. Hence $|E(M)| \geqslant|E(N)|+2$. As $|E(N)| \geqslant 4$, we deduce that $|E(M)| \geqslant 6$.

We know that $M$ has $N$ as an s-minor. This means, of course, that $N$ can be obtained from $M$ by a sequence of contractions, deletions accompanied by compactifications, and series compressions. Our first goal will be to prove the following.

Lemma 56. The 2-polymatroid $M$ has an s-minor that is isomorphic to $N$ such that, in the production of this s-minor, all of the series compressions are done last in the process.

Next we focus on the c-minor $N_{0}$ of $M$ that is obtained in the above process after all of the contractions and compactified deletions are done but before doing any of the series compressions. By Lemma 19, $N_{0}$ is 3 -connected. In view of this, we see that, to prove Theorem 4 , it suffices to prove Theorem 5 , which we restate here for the reader's convenience. 
Theorem 57. Let $M$ and $N$ be distinct 3-connected 2-polymatroids such that $N$ is a c-minor of $M$ and $|E(N)| \geqslant 4$. Then

(i) $r(M) \geqslant 3$ and $M$ is a whirl or the cycle matroid of a wheel; or

(ii) $M$ has an element $\ell$ such that $M \backslash \ell$ or $M / \ell$ is 3-connected having a c-minor isomorphic to $N$; or

(iii) $M$ has a prickly 3-separator $\{y, z\}$ such that $M \downarrow y$ is 3-connected having a c-minor isomorphic to $N$.

Note that this restatement of Theorem 5 omits mention of $M \downarrow z$ in (iii) since, by Lemma 16, $M \downarrow z \cong M \downarrow j$. Our focus now becomes proving Theorem 57 . For the rest of this section, we assume that the pair $(M, N)$ is a counterexample to that theorem. Note that, by Lemma 22, this means that $\left(M^{*}, N^{*}\right)$ is also a counterexample to Theorem 57 . The first two steps in the argument, whose proofs appear in Section 7, are as follows.

Lemma 58. $M$ has no point $z$ such that both $M \rrbracket z$ and $M / z$ have c-minors isomorphic to $N$.

Lemma 59. $M$ has no element $\ell$ such that $M \backslash \ell$ or $M / \ell$ is disconnected having a c-minor isomorphic to $N$.

Note that the use of $\ell$ above, and in what follows, does not imply that $\ell$ is a line, although most of our attention will be focused on that case.

Now $N$ occurs as a c-minor of $M$. Although we will often work with c-minors of $M$ that are isomorphic to $N$, at a certain point in the argument, we will settle on a particular labelled c-minor of $M$ that is isomorphic to $N$.

When $M$ has $N$ as a c-minor and has a 2-separation $(X, Y)$, either $X$ or $Y$, say $X$, contains at least $|E(N)|-1$ elements of $N$. We call $X$ the $N$-side of the 2-separation and $Y$ the $n o n-N$-side.

Suppose $M \rrbracket \ell$ has $N$ as a c-minor. As the theorem fails, $M \backslash \ell$ is not 3-connected. Now, by Lemma 2(iii), $\lambda_{M \backslash \ell}=\lambda_{M \backslash \ell}$. Thus a partition $(X, Y)$ of $E-\ell$ with $\min \{|X|,|Y|\} \geqslant 2$ is a 2-separation of $M \backslash \ell$ if and only if it is a 2-separation of $M \backslash \ell$. It follows that we can label the $N$ - and non- $N$-sides of a non-trivial 2-separation of $M \backslash \ell$ based on their labels in the corresponding 2-separation of $M \rrbracket \ell$. Among all 2-separations of $M \backslash \ell$, let the maximum cardinality of the non- $N$-side be $\mu(\ell)$. Similarly, if $M / \ell$ has $N$ as a c-minor, let $\mu^{*}(\ell)$ be the maximum cardinality of the non- $N$-side of a 2-separation of $M / \ell$. We observe that $\mu(\ell)$ and $\mu^{*}(\ell)$ are not defined unless $M \backslash \ell$ and $M / \ell$, respectively, have $N$ as a c-minor.

The next step in the argument establishes the following.

60. $M$ has no element $\ell$ for which $\mu(\ell)=2$ or $\mu^{*}(\ell)=2$.

The argument for 60 is quite long since it involves a detailed analysis of the various structures that can arise on the non- $N$-side when $\mu(\ell)=2$. We then use duality to eliminate the cases when $\mu^{*}(\ell)=2$. These arguments appear in Section 8 . 
Recall that a special $N$-minor of $M$ is any c-minor of $M$ that is either equal to $N$ or differs from $N$ by having a single point relabelled. The next major step in the argument, which is dealt with in Lemma 93, proves the following.

61. If $(X, Y)$ is a 2-separation of $M \backslash \ell$ where $X$ is the $N$-side and $|Y|=\mu(\ell)$, then $Y$ contains an element $y$ such that both $M \backslash y$ and $M / y$ have special $N$-minors.

We say that an element $\ell$ of $M$ is doubly labelled if both $M \backslash \ell$ and $M / \ell$ have special $N$-minors. In Lemma 96, we use the doubly labelled element found in the last step to prove the following.

62. There is a c-minor $N^{\prime}$ of $M$ that is isomorphic to $N$ such that $M$ has a 3 -separator $(X, Y)$ with $\left|E\left(N^{\prime}\right) \cap Y\right| \leqslant 1$ such that if $|Y|=2$, then both elements of $Y$ are lines.

The particular c-minor $N^{\prime}$ whose existence is proved in 62 is the one used throughout the rest of the argument. From that point on in the argument, we use $N$ to denote $N^{\prime}$. An exactly 3-separating set $Y$ is called a non- $N$-3-separator if $|E(N) \cap Y| \leqslant 1$ and, when $|Y|=2$, both elements of $Y$ are lines. By 62, a non- $N$-3-separator exists. Hence there is a minimal such set.

At the beginning of Section 11, we prove that

63. $M$ has a minimal non- $N$-3-separator with at least three elements.

The rest of Section 11 is devoted to showing the following.

64. A minimal non- $N$-3-separator of $M$ with exactly three elements consists of three lines.

The purpose of Section 12 is to prove that

65. $M$ has a minimal non- $N$-3-separator with at least four elements.

The argument to show 65 is quite long since it involves treating all non- $N$-3-separators that consist of exactly three lines.

The next step, which is shown in Section 13, establishes the following.

66 . If $Y_{1}$ is a minimal non- $N$-3-separator of $M$ with at least four elements, then $Y_{1}$ contains a doubly labelled element.

Next we take the doubly labelled element $\ell$ identified in the last step. We then take non-trivial 2-separations $\left(D_{1}, D_{2}\right)$ and $\left(C_{1}, C_{2}\right)$ of $M \backslash \ell$ and $M / \ell$, respectively, having $D_{1}$ and $C_{1}$ as their $N$-sides. We show that these 2-separations can be chosen so that each of $D_{2}$ and $C_{2}$ is contained in $Y_{1}-\ell$, and neither contains any points of $M$.

We then show that each of $D_{1} \cap C_{2}, D_{2} \cap C_{1}$, and $D_{2} \cap C_{2}$ consists of a single line of $M$, that the union of these lines spans $\ell$, and these four lines together make up $Y_{1}$.

The final contradiction is obtained by showing that $M / \ell_{22}$ is 3 -connected having a c-minor isomorphic to $N$, where $\ell_{22}$ is the unique element in $D_{2} \cap C_{2}$.

\section{The reduction to c-minors}

The goal of this section is to prove Lemma 56 and thereby show that Theorem 4 can be proved by verifying Theorem 57 . 
Proof of Lemma 56. Consider the s-minors of $M$ that are isomorphic to $N$ and are obtained using the minimum number of series compressions. Suppose $N_{1}$ is such an s-minor and let the number of series compressions used in its production be $m$. If $m=0$, then $N_{1}$ is an s-minor of $M$ satisfying the requirements of the lemma. Hence we may assume that $m>0$. Let $n_{1}$ be the number of elements that are removed, via deletion or contraction, after the last series compression has been completed. For $2 \leqslant i \leqslant m$, let $n_{i}$ be the number of elements that are removed, via deletion or contraction, between the $(m-i+1)$ st and the $(m-i+2)$ nd series compressions. Consider the sequence $\left(n_{1}, n_{2}, \ldots, n_{m}\right)$ and let $N_{0}$ be a choice for $N_{1}$ for which the corresponding sequence is lexicographically minimal. If each $n_{i}$ is zero, then we have found, as desired, an s-minor of $M$ in which all of the series compressions are performed after all of the contractions and compactified deletions. Assume then that $n_{i}$ is the first non-zero $n_{j}$. Let $P$ be the 2-polymatroid that we have immediately prior to the $(m-i+1)$ st series compression, with this series compression involving compressing the line $k$ from the prickly 3 -separator $\{j, k\}$ of $P$. Let $Q$ be the 2 -polymatroid we have immediately prior to the $(m-i+2)$ nd series compression.

By Lemma 55, we may assume that $j$ is neither deleted or contracted in producing $N_{0}$ otherwise we can replace the compression of $k$ by a deletion followed by a compactification or by a contraction. By Lemma 10, we may assume that either

(a) all of the elements removed in producing $Q$ from $P \downarrow k$ are done so by deletion followed by compactification; or

(b) the next move in the production of $Q$ is the contraction of an element, say $y$.

Assume that (b) holds. By Lemma 18, $P \downarrow k / y=P / y \downarrow k$. Assume that $\{j, k\}$ is not a prickly 3-separator of $P / y$. We now apply Lemma 53. If $r(\{y, j, k\})=3$, then $j$ is a loop of $P \downarrow k / y$ so $j$ must be deleted or contracted to produce $N_{0}$, a contradiction. If $P \downarrow k / y$ is $P / y \rrbracket k$ or $P / y / k$, then we do not need to compress $k$ in the production of $N_{0}$, so the choice of $N_{0}$ is contradicted. We are left with the possibility that $P \downarrow k / y$ can be obtained from $P / y \| j$ by relabelling $k$ as $j$. Again we obtain the contradiction that we can reduce the number of series compressions where, if $j \in E\left(N_{0}\right)$, we replace $N_{0}$ by the 2-polymatroid in which $j$ is relabelled by $k$. We conclude that $\{j, k\}$ is a prickly 3 -separator of $P / y$. In that case, interchanging the compression of $k$ and the contraction of $y$ in $P$ produces a 2-polymatroid in which $n_{i}$ is reduced and so the choice of $N_{0}$ is contradicted. We deduce that (b) does not hold, so (a) holds.

In the construction of $N_{0}$, let $y$ be the first element that is deleted following the compression of $k$. Now, by Lemma 18, $P \downarrow k \backslash y=(P \downarrow k \backslash y)^{b}=(P \backslash y \downarrow k)^{b}$. By Lemmas 2 and 17, $P$ is compact, so $r(E-y)=r(E)$ where $E$ is the ground set of $P$. If $r(E-\{y, j, k\})=r(E)-3$, then $P \backslash y$ has $\{j, k\}$ as a 1 -separating set. This is a contradiction as $j$ cannot be deleted or contracted in the production of $N_{0}$ from $P$. Hence

$$
r(E-\{y, j, k\}) \geqslant r(E)-2 .
$$

Next we show that

66.1. $r_{P \backslash y \downarrow k}(E-\{y, j, k\})=r(E-\{y, j, k\})$. 
By Lemmas 55(i) and 18(i), $P \downarrow k \backslash j=P \backslash k, j$ and $P \downarrow k \backslash y=P \backslash y \downarrow k$. Thus

$$
P \backslash k \backslash j \backslash y=P \downarrow k \backslash j \backslash y=P \downarrow k \backslash y \backslash j=P \backslash y \downarrow k \backslash j,
$$

and 66.1 follows.

By definition,

$$
r_{P \backslash y \downarrow k}(E-\{y, k\})= \begin{cases}r(E-\{y, k\}) & \text { if } r(E-y)>r(E-\{y, k\}) ; \\ r(E-\{y, k\})-1 & \text { if } r(E-y)=r(E-\{y, k\}) .\end{cases}
$$

But $r(E)=r(E-y)$ and, by submodularity, $r(E-\{y, k\}) \geqslant r(E-y)-1$. Thus, if $r(E-y)>r(E-\{y, k\})$, then $r(E-\{y, k\})=r(E)-1$; and if $r(E-y)=r(E-\{y, k\})$, then $r(E-\{y, k\})-1=r(E)-1$. Hence

$$
r(P \backslash y \downarrow k)=r(E)-1 .
$$

We now show that

66.2. $\lambda_{P \backslash y}(\{j, k\})=\lambda_{P \backslash y \downarrow k}(\{j\})$.

Using 66.1 and (13), we see that

$$
\begin{aligned}
\lambda_{P \backslash y}(\{j, k\}) & =r(\{j, k\})+r(E-\{y, j, k\})-r(E-y) \\
& =3+r_{P \backslash y \downarrow k}(E-\{y, j, k\})-r(E) \\
& =2+r_{P \backslash y \downarrow k}(E-\{y, j, k\})-(r(E)-1) \\
& =r_{P \backslash y \downarrow k}(\{j\})+r_{P \backslash y \downarrow k}(E-\{y, j, k\})-r(P \backslash y \downarrow k) \\
& =\lambda_{P \backslash y \downarrow k}(\{j\}) .
\end{aligned}
$$

Thus 66.2 holds.

Let $\left\{\ell_{1}, \ell_{2}, \ldots, \ell_{t}\right\}$ be the set $S$ of 2 -separating lines in $P \backslash y$. Clearly no member of $S-k$ is parallel to $k$. We show next that

66.3. $S \cap\{j, k\} \neq \emptyset$.

Suppose, instead, that neither $j$ nor $k$ is in $S$. Then, by Lemma 15 , the set of 2 separating lines of $P \backslash y \downarrow k$ is either $S$ or $S \cup j$. Assume the latter. Then, by 66.2, $\lambda_{P \backslash y}(\{j, k\})=1$. Thus $P \backslash y$ is the 2 -sum with basepoint $p$ of two 2-polymatroids $P_{1}$ and $P_{2}$ having ground sets $(E-\{y, j, k\}) \cup p$ and $\{j, k, p\}$, respectively. Since neither $j$ nor $k$ is 2-separating in $P \backslash y$, it follows that, in the rank-three 2-polymatroid $P_{2}$, the point $p$ does not lie on either of the lines $j$ or $k$. By Lemma 46(vi), $P \backslash y \downarrow k=P_{1} \oplus_{2}\left(P_{2} \downarrow k\right)$. Now $P_{2} \downarrow k$ consists of the line $j$ with the point $p$ lying on it. As $S \cup j$ is the set of 2-separating lines of $P \backslash y \downarrow k$, and $P_{2} \downarrow k \downarrow \downarrow j=P_{2} / k$, we deduce that $P \backslash y \downarrow k \downarrow j=P \backslash y / k$. 
It follows that $S$ is the set of 2 -separating lines of $P \backslash y / k$. Thus

$$
\begin{aligned}
& P \downarrow k \backslash y=(P \backslash y \downarrow k)^{b} \\
& =P \backslash y \downarrow k \downarrow j \downarrow \\
& =(P \backslash y \downarrow k \downarrow j) \downarrow \ell_{1} \downarrow \ell_{2} \downarrow \ldots \downarrow
\end{aligned}
$$

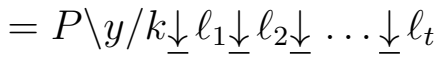

$$
\begin{aligned}
& =P / k \backslash y \downarrow \ell_{1} \ell_{2} \ell_{2} \ldots \ell_{t} \\
& =P / k \rrbracket y \text {. }
\end{aligned}
$$

We conclude that, instead of compressing $k$, we can contract it, which contradicts that choice of $N_{0}$.

We may now assume that $S$ is the set of 2-separating lines of $P \backslash y \downarrow k$. Then, by 66.2 , $\lambda_{P \backslash y}(\{j, k\})=2$. Now

$$
P \backslash y \downarrow k=P \backslash y \underline{\downarrow} \ell_{1} \downarrow \ell_{2} \downarrow \cdots \underline{\downarrow} \ell_{t} \downarrow k .
$$

Thus, by repeated application of Lemma 52 and using Lemma 18, we see that

$$
\begin{aligned}
P \backslash y \downarrow k & =P \backslash y \downarrow k \downarrow \ell_{1} \downarrow \ell_{2} \downarrow \cdots \underline{\downarrow} \ell_{t} \\
& =P \downarrow k \backslash y \downarrow \ell_{1} \downarrow \ell_{2} \downarrow \cdots \underline{\downarrow} \ell_{t} \\
& =P \downarrow k \backslash y .
\end{aligned}
$$

Since neither $j$ nor $k$ is in $S$, we see that $\sqcap(\{j\}, E-\{y, j, k\})=1=\sqcap(\{k\}, E-\{y, j, k\})$. Thus $\{j, k\}$ is a prickly 3-separator of $P \backslash y$. It follows without difficulty that $\{j, k\}$ is a prickly 3-separator of $P \Downarrow y$. As $P \downarrow k \| y=P \Downarrow y \downarrow k$, we can reduce $n_{i}$, a contradiction. We conclude that 66.3 holds.

We now know that $j$ or $k$ is in $S$. Suppose next that both $j$ and $k$ are in $S$. Thus $r(E-\{y, j\})=r(E)-1=r(E-\{y, k\})$. By submodularity and (12), we deduce that $r(E-\{y, j, k\})=r(E)-2$. Hence $P \backslash y$ is the 2-sum with basepoint $p$ of two 2-polymatroids $P_{1}$ and $P_{2}$ having ground sets $(E-\{y, j, k\}) \cup p$ and $\{j, k, p\}$, respectively. Moreover, in $P_{2}$, the point $p$ lies on both $j$ and $k$. Now $P \| y=P \backslash y \underline{\downarrow} \ell_{1} \downarrow \ell_{2} \downarrow \cdots \underline{\downarrow} \ell_{t} \underline{j} \underline{\downarrow} k$. Hence $P \| y$ has $j$ and $k$ as parallel points. Thus

$$
\begin{aligned}
& P \downarrow k \backslash y=(P \backslash y \downarrow k)^{b} \\
& =P \backslash y \downarrow k \downarrow \underline{\downarrow} \ell_{1} \underline{\ell_{2}} \underline{\downarrow} \ldots \underline{\downarrow} \ell_{t} \underline{j} \\
& =P \backslash y \underline{\downarrow} \ell_{1} \downarrow \ell_{2} \downarrow \cdots \downarrow-\ell_{t} \downarrow j \downarrow k \quad \text { by Lemma } 52 \text {; }
\end{aligned}
$$

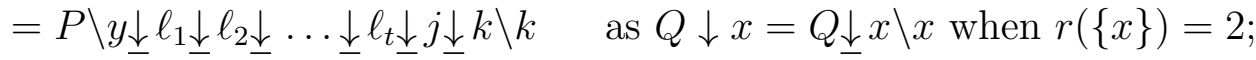

$$
\begin{aligned}
& =P \Downarrow y \backslash k \\
& =P \Downarrow y \rrbracket k \text {, }
\end{aligned}
$$

where the last step follows because $P \downarrow k \Downarrow y$ is compact and so $P \Downarrow y \backslash k$ is compact. Again we have a contradiction since we have managed to remove $k$ via deletion rather than by series compression. 
Now assume that $k$ is in $S$ but $j$ is not. Then

$$
\begin{aligned}
& P \downarrow k \rrbracket y=(P \backslash y \downarrow k)^{b} \\
& =P \backslash y \underline{\downarrow} \ell_{1} \downarrow \ell_{2} \downarrow \cdots \underline{\downarrow} \ell_{t} \downarrow k \\
& =P \backslash y \underline{\downarrow} \ell_{1} \downarrow \ell_{2} \downarrow \cdots \downarrow \\
& =P \Downarrow \backslash y \backslash k \\
& =P \Downarrow y \rrbracket k \text {. }
\end{aligned}
$$

Once again we have managed to avoid the need to perform a series compression on $k$, a contradiction.

Finally, suppose $j$ is in $S$ but $k$ is not. Then we use the fact, established in Lemma 16, that $P \downarrow j$ is $P \downarrow k$ with $j$ relabelled as $k$. The argument in the last paragraph yields a contradiction where, when $j \in E\left(N_{0}\right)$, we replace $N_{0}$ by the 2-polymatroid in which $j$ is relabelled as $k$.

\section{$7 \quad$ Eliminating doubly labelled points}

In this section, we prove that, when $(M, N)$ is a counterexample to Theorem $57, M$ has no doubly labelled point and has no element whose deletion or contraction is disconnected having a c-minor isomorphic to $N$.

The following elementary lemmas will be helpful.

Lemma 67. Let $T$ be a set of three points in a 2-polymatroid $Q$ and suppose $x \in T$.

(i) If $T$ is a triangle of $Q$, then $\lambda_{Q / x}(T-x) \leqslant 1$.

(ii) If $T$ is a triad of $Q$, then $\lambda_{Q \backslash x}(T-x) \leqslant 1$.

Lemma 68. Let $T_{1}$ and $T_{2}$ be distinct triads in a 2-polymatroid $Q$. Then $r\left(E(Q)-\left(T_{1} \cup\right.\right.$ $\left.\left.T_{2}\right)\right) \leqslant r(Q)-2$.

Proof. We know that $r\left(E(Q)-T_{i}\right)=r(Q)-1$ for each $i$. The lemma follows easily by applying the submodularity of the rank function.

Proof of Lemma 58. Suppose $M$ has a point $z$ such that both $M \rrbracket z$ and $M / z$ have cminors isomorphic to $N$. Then neither $M \backslash z$ nor $M / z$ is 3-connected. We may also assume that $M$ is neither a whirl nor the cycle matroid of a wheel. By Lemma 41, $M$ has points $s$ and $t$ such that $\{z, s, t\}$ is a triangle or a triad of $M$. By replacing $M$ by $M^{*}$ if necessary, we may assume that $\{z, s, t\}$ is a triangle of $M$. Then $M / z$ has $s$ and $t$ as a pair of parallel points. Thus both $M / z \backslash s$ and $M / z \backslash t$ have c-minors isomorphic to $N$. As the theorem fails, neither $M \backslash s$ nor $M \backslash t$ is 3-connected. Thus, by Lemma 42, $M$ has a triad that contains $z$ and exactly one of $s$ and $t$. We may assume that the triad is $\{z, s, u\}$. Then $t, z, s, u$ is a fan in $M$.

Now take a fan $x_{1}, x_{2}, \ldots, x_{k}$ in $M$ of maximal length such that both $M \backslash x_{2}$ and $M / x_{2}$ have c-minors isomorphic to $N$. Then $k \geqslant 4$. A straightforward induction argument, whose details we omit, gives the following. 
68.1. For all $i$ in $\{2,3, \ldots, k-1\}$, both $M \backslash x_{i}$ and $M / x_{i}$ have c-minors isomorphic to $N$.

Now consider $\left\{x_{k-2}, x_{k-1}, x_{k}\right\}$. Suppose first that it is a triangle. As $M / x_{k-1}$ has a c-minor isomorphic to $N$, so do $M / x_{k-1} \backslash x_{k}$ and hence $M \backslash x_{k}$. As $M \backslash x_{k-1}$ also has a cminor isomorphic to $N$, neither $M \backslash x_{k}$ nor $M \backslash x_{k-1}$ is 3-connected. Thus, by Lemma 42, it follows that $M$ has a triad $T^{*}$ containing $x_{k}$ and exactly one of $x_{k-2}$ and $x_{k-1}$. Let its third element be $x_{k+1}$. By the choice of $k$, it follows that $x_{k+1} \in\left\{x_{1}, x_{2}, \ldots, x_{k-3}\right\}$. Suppose $k=4$. Then $x_{1} \in T^{*}$. Then $\left\{x_{1}, x_{2}, x_{3}, x_{4}\right\}$ contains two distinct triads so, by Lemma 68 , $r\left(E-\left\{x_{1}, x_{2}, x_{3}, x_{4}\right\}\right) \leqslant r(M)-2$. Thus $\lambda\left(\left\{x_{1}, x_{2}, x_{3}, x_{4}\right\}\right) \leqslant 1$, a contradiction since $|E| \geqslant 6$. We deduce that $k \geqslant 5$.

As $M$ cannot have a triangle and a triad that meet in a single element, either

(i) $x_{k+1}=x_{1}$ and $\left\{x_{1}, x_{2}, x_{3}\right\}$ is a triad; or

(ii) $T^{*}$ contains $\left\{x_{k}, x_{k-2}\right\}$, and $x_{k+1} \in\left\{x_{k-3}, x_{k-4}\right\}$.

In the latter case, let $X=\left\{x_{k-4}, x_{k-3}, x_{k-2}, x_{k-1}, x_{k}\right\}$. Then, by Lemma 68 ,

$$
r(X)+r(E-X)-r(M) \leqslant 3+r(M)-2-r(M)=1 .
$$

Since $M$ is 3 -connected, we obtain a contradiction unless $E-X$ is empty or contains a single element, which must be a point. In the exceptional case, $M$ is a 3-connected matroid having 5 or 6 elements and containing a 5 -element subset that contains two triangles and two triads. But there is no 3-connected matroid with these properties. We deduce that (ii) does not hold.

We now know that (i) holds and that $T^{*}$ contains $\left\{x_{k}, x_{k-1}\right\}$. Then $k$ is even. Let $X=\left\{x_{1}, x_{2}, \ldots, x_{k}\right\}$. As $M \backslash x_{2}$ has a c-minor isomorphic to $N$ and has $\left\{x_{1}, x_{3}\right\}$ as a series pair of points, it follows that $M \backslash x_{2} / x_{1}$, and hence, $M / x_{1}$ has a c-minor isomorphic to $N$. Thus, by Lemma $42, M$ has a triangle containing $x_{1}$ and exactly one of $x_{2}$ and $x_{3}$. This triangle must also contain $x_{k}$ or $x_{k-1}$. Hence $r(X) \leqslant r\left(\left\{x_{2}, x_{4}, x_{6}, \ldots, x_{k}\right\}\right) \leqslant \frac{k}{2}$. Also $r^{*}(X) \leqslant r\left(\left\{x_{1}, x_{3}, x_{5}, \ldots, x_{k-1}\right\}\right) \leqslant \frac{k}{2}$. Thus, by Lemma $28, \lambda(X)=0$, so $X=E(M)$. Hence $M$ is a 3-connected matroid in which every element is in both a triangle and a triad, so $M$ is a whirl or the cycle matroid of a wheel, a contradiction.

We still need to consider the case when $\left\{x_{k-2}, x_{k-1}, x_{k}\right\}$ is a triad of $M$. Then it is a triangle of $M^{*}$ and the result follows by replacing $M$ by $M^{*}$ in the argument above.

Proof of Lemma 59. Suppose $M \backslash \ell$ is disconnected having a c-minor isomorphic to $N$. Then $E(M \backslash \ell)$ has a non-empty proper subset $X$ such that $\lambda_{M \backslash \ell}(X)=0$ and $M \backslash \ell \backslash X$ has $N$ as a c-minor. Then, by Lemma 58, every element of $X$ must be a line. Let $Y=E(M \backslash \ell)-X$. Since $r(M \backslash \ell)=r(M)$, we deduce that

$$
r(X)+r(Y)=r(M) .
$$

As $r(X) \geqslant 2$ and $(X, Y \cup \ell)$ is not a 2-separation of $M$, we deduce that $r(Y \cup \ell)=r(Y)+2$. It follows, since $Y$ and $\ell$ are skew and $M \backslash X$ has $N$ as a c-minor, that $M / \ell$ has $N$ as a 
c-minor. Since $(M, N)$ is a counterexample to the theorem, $M / \ell$ is not 3 -connected. Thus there is a partition $\left(C_{1}, C_{2}\right)$ of $E(M)-\ell$ such that, for some $k$ in $\{1,2\}$,

$$
r_{M / \ell}\left(C_{1}\right)+r_{M / \ell}\left(C_{2}\right) \leqslant r(M / \ell)+k-1
$$

where, if $k=2$, we may assume that $\min \left\{\left|C_{1}\right|, r_{M / \ell}\left(C_{1}\right),\left|C_{2}\right|, r_{M / \ell}\left(C_{2}\right)\right\} \geqslant 2$. Hence

$$
r\left(C_{1} \cup \ell\right)+r\left(C_{2} \cup \ell\right) \leqslant r(M)+3 .
$$

By (14), (16), and submodularity, $r\left(X \cup C_{1} \cup \ell\right)+r\left(X \cap C_{1}\right)+r\left(Y \cup C_{2} \cup \ell\right)+r\left(Y \cap C_{2}\right) \leqslant$ $2 r(M)+3$. Then

$$
\begin{gathered}
r\left(X \cup C_{1} \cup \ell\right)+r\left(Y \cap C_{2}\right) \leqslant r(M)+1 \quad \text { or } \\
r\left(Y \cup C_{2} \cup \ell\right)+r\left(X \cap C_{1}\right) \leqslant r(M)+1,
\end{gathered}
$$

SO

$$
r\left(Y \cap C_{2}\right) \leqslant 1 \text { or } r\left(X \cap C_{1}\right) \leqslant 1 .
$$

By symmetry,

$$
r\left(Y \cap C_{1}\right) \leqslant 1 \text { or } r\left(X \cap C_{2}\right) \leqslant 1 .
$$

Since $X$ does not contain any points, either $r\left(Y \cap C_{2}\right) \leqslant 1$ and $r\left(Y \cap C_{1}\right) \leqslant 1$; or, for some $i$ in $\{1,2\}$,

$$
X \cap C_{i}=\emptyset \quad \text { and } \quad r\left(Y \cap C_{i}\right) \leqslant 1 .
$$

In the former case, $|Y| \leqslant 2$, a contradiction since $Y$ contains $E(N)$. In the latter case, we may assume that $C_{1}$ consists of a single point $p$. Then we deduce that $k=1$ in (15). Thus $p$ is a point of $M$ and $\{p\}$ is a component of $M / \ell$. Hence both $M \backslash p$ and $M / p$ have $N$ as c-minors, a contradiction to Lemma 58. We conclude that if $M \backslash \ell$ has a c-minor isomorphic to $N$, then $M \backslash \ell$ is 2-connected.

Now suppose $M / \ell$ is disconnected having a c-minor isomorphic to $N$. By Lemma 2,

$$
\lambda_{M / \ell}=\lambda_{(M / \ell)^{*}}=\lambda_{\left(M^{*} \backslash \ell\right)^{b}}=\lambda_{M^{*} \backslash \ell} .
$$

Thus, by replacing $M$ by $M^{*}$ in the argument above, we deduce that if $M / \ell$ has a c-minor isomorphic to $N$, then $M / \ell$ is 2 -connected.

\section{If all 2-separations have a side with at most two elements}

The purpose of this section is to treat 60 . The argument here is long as it involves analyzing numerous cases. The setup is that $M$ and $N$ are 3-connected 2-polymatroids such that $|E(N)| \geqslant 4$. The pair $(M, N)$ is a counterexample to Theorem 57 and $M$ has an element $\ell$ such that $M \backslash \ell$ has $N$ as a c-minor. Thus $M \backslash \ell$ is not 3-connected. We assume that the non- $N$-side of every non-trivial 2-separation of $M \backslash \ell$ has exactly two elements. Thus $\mu(\ell)=2$. Let $(X, Y)$ be a non-trivial 2-separation of $M \backslash \ell$ in which $Y$ is the non- $N$ side. Now $M \backslash \ell$ can be written as the 2-sum, with basepoint $p$, of 2-polymatroids $M_{X}$ and $M_{Y}$ having ground sets $X \cup p$ and $Y \cup p$. The first lemma identifies the various possibilities for $M_{Y}$. 
Lemma 69. Let $P$ be a 2-connected 2-polymatroid with three elements and rank at least two. Suppose $P$ has a distinguished point $p$. Then $P$ is one of the nine 2-polymatroids, $P_{1}, P_{2}, \ldots, P_{9}$, depicted in Figure 1.

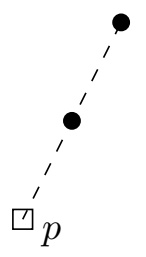

$P_{1}$

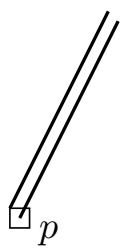

$P_{4}$

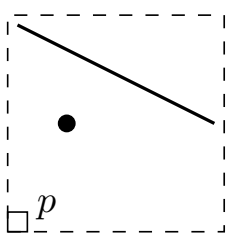

$P_{7}$

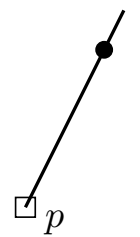

$P_{2}$

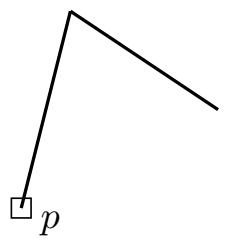

$P_{5}$

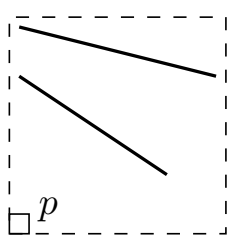

$P_{8}$

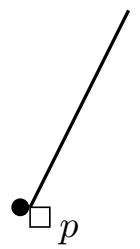

$P_{3}$

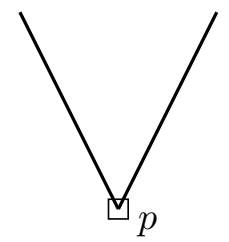

$P_{6}$

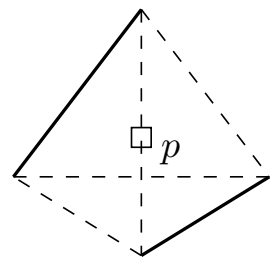

$P_{9}$

Figure 1: The nine possible 3-element 2-polymatroids in Lemma 69.

Proof. As $P$ is 2 -connected having rank at least 2 , we see that $2 \leqslant r(P) \leqslant 4$. If $r(P)=2$, then $P$ is one of $P_{1}, P_{2}, P_{3}$, or $P_{4}$; if $r(P)=3$, then $P$ is one of $P_{5}, P_{6}, P_{7}$, or $P_{8}$; if $r(P)=4$, then $P$ is $P_{9}$.

We shall systematically eliminate the various possibilities for $M_{Y}$. In each case, we will label the two elements of $M_{Y}$ other than $p$ by $a$ and $b$.

Lemma 70. $M_{Y}$ is not isomorphic to $P_{2}$ or $P_{3}$.

Proof. Assume the contrary. Then $M_{Y}$ and hence $M$ has a point $q$ on a line $y$ where $q \neq p$. Thus $M \backslash q$ is 3-connected, so $M \backslash q$ has no c-minor isomorphic to $N$. In particular, $q \in E(N)$. Thus $y \notin E(N)$. As $M \backslash \ell / y$ has $q$ as a loop, we must delete $y$ from $M \backslash \ell$ in constructing $N$. If $P=P_{2}$, then $M \backslash \ell \backslash y$ has $\{q\}$ as a 1-separating set, a contradiction. Thus $P=P_{3}$. Then, in $M \Downarrow \ell$, we see that $y$ and $q$ are parallel points. Thus $M \backslash \ell \backslash q$, and hence $M \backslash q$, has a c-minor isomorphic to $N$, a contradiction. 
Lemma 71. $M_{Y}$ is not isomorphic to $P_{4}$.

Proof. Assume the contrary. Let the two parallel lines in $M_{Y}$ be $y$ and $y^{\prime}$ where we may assume that $y \notin E(N)$. Now $M \backslash y$ is 3-connected, so $M \backslash y$ does not have $N$ as a c-minor. Thus $M / y$ has $N$ as a c-minor. But $y^{\prime}$ is a loop of $M / y$, so $y^{\prime} \notin E(N)$ and $M \backslash y^{\prime}$ has $N$ as a c-minor. Since $M \backslash y^{\prime}$ is 3 -connected, we have a contradiction.

The next lemma is designed to facilitate the elimination of the cases when $M_{Y}$ is one of $P_{1}, P_{7}$, or $P_{9}$.

Lemma 72. Suppose both $a$ and $b$ are skew to $p$ in $M_{Y}$, and both $M_{Y} / a$ and $M_{Y} / b$ are 2-connected. Then $M / a$ and $M / b$ have 2 -separations $\left(X_{a}, Y_{a}\right)$ and $\left(X_{b}, Y_{b}\right)$ such that $\ell \in Y_{a} \cap Y_{b}$. Moreover, both $M / a$ and $M / b$ have special $N$-minors, and

(i) $b \in X_{a}$ and $a \in X_{b}$;

(ii) both $Y_{a}$ and $Y_{b}$ properly contain $\{\ell\}$;

(iii) $\left(X_{a}, Y_{a}-\ell\right)$ and $\left(X_{b}, Y_{b}-\ell\right)$ are 2-separating partitions of $M / a \backslash \ell$ and $M / b \backslash \ell$, respectively, and $\ell \in \mathrm{cl}_{M / a}\left(Y_{a}-\ell\right)$ and $\ell \in \mathrm{cl}_{M / b}\left(Y_{b}-\ell\right)$;

(iv) $\left(X_{a} \cup a, Y_{a}-\ell\right)$ and $\left(X_{b} \cup b, Y_{b}-\ell\right)$ are 2-separating partitions of $M \backslash \ell$;

(v) for $c$ in $\{a, b\}$, provided $a$ or $b$ is a point, $\left(X_{c}, Y_{c}-\ell\right)$ is a 2-separation of $M / c \backslash \ell$ and $\left(X_{c} \cup c, Y_{c}-\ell\right)$ is a 2-separation of $M \backslash \ell$;

(vi) either $\left(Y_{a}-\ell\right) \cap\left(Y_{b}-\ell\right) \neq \emptyset$; or each of $X_{b} \cap\left(Y_{a}-\ell\right)$ and $X_{a} \cap\left(Y_{b}-\ell\right)$ consists of a single point, both $a$ and $b$ are lines of $M$, and, when $r(\{a, b\})=4$, the element $\ell$ is a point of $M$.

Proof. Since both $M_{Y} / a$ and $M_{Y} / b$ are 2-connected, it follows by Lemma 51 that both $M \backslash \ell / a$ and $M \backslash \ell / b$ have special $N$-minors. Hence so do both $M / a$ and $M / b$. Since the theorem fails, $M / a$ and $M / b$ have 2-separations $\left(X_{a}, Y_{a}\right)$ and $\left(X_{b}, Y_{b}\right)$ such that $\ell \in Y_{a} \cap Y_{b}$.

To see that (i) holds, it suffices to show that $b \in X_{a}$. Assume $b \in Y_{a}$. Then

$$
r_{M / a}\left(X_{a}\right)+r_{M / a}\left(Y_{a}\right)=r(M / a)+1,
$$

so $r_{M}\left(X_{a} \cup a\right)-r_{M}(\{a\})+r_{M}\left(Y_{a} \cup a\right)=r(M)+1$. As $a$ is skew to $p$ in $M_{Y}$, it follows that $a$ is skew to $X$ in $M$. Since $X_{a} \subseteq X$, it follows that $\left(X_{a}, Y_{a} \cup a\right)$ is a 2-separation of $M$, a contradiction. Hence (i) holds.

Part (ii) is an immediate consequence of Lemma 37. To prove (iii), first observe that, by Proposition $47, M / a \backslash \ell$ is 2 -connected. We show next that

$$
r(M / a \backslash \ell)=r(M / a) .
$$

Suppose not. Then $r(M / a \backslash \ell) \leqslant r(M / a)-1$. Since $M / a$ is 2-connected, it follows that equality must hold here and $\ell$ is a line of $M / a$. This gives a contradiction to Lemma 37 . Hence (17) holds. 
Now

$$
\begin{aligned}
r(M / a \backslash \ell)+1 & \leqslant r_{M / a \backslash \ell}\left(X_{a}\right)+r_{M / a \backslash \ell}\left(Y_{a}-\ell\right) \\
& \leqslant r_{M / a}\left(X_{a}\right)+r_{M / a}\left(Y_{a}\right) \\
& =r(M / a)+1 \\
& =r(M / a \backslash \ell)+1,
\end{aligned}
$$

where the last equality follows from (17). We see that equality must hold throughout the last chain of inequalities. Hence $\left(X_{a}, Y_{a}-\ell\right)$ is a 2-separating partition of $M / a \backslash \ell$, and $\ell \in \mathrm{cl}_{M / a}\left(Y_{a}-\ell\right)$. Using symmetry, we deduce that (iii) holds.

Since $b \in X_{a}$, we see that $Y_{a}-\ell \subseteq X$, so $a$ is skew to $Y_{a}-\ell$. It follows by (iii) that $\left(X_{a} \cup a, Y_{a}-\ell\right)$ is a 2-separating partition of $M \backslash \ell$, and (iv) follows by symmetry.

To show (v), observe that, since $Y_{c}-\ell$ avoids $\{a, b\}$, it follows that $c$ is skew to $Y_{c}-\ell$. Thus it suffices to show that $\left(X_{c}, Y_{c}-\ell\right)$ is a 2-separation of $M / c \backslash \ell$. Assume it is not. Then $Y_{c}-\ell$ consists of a single point $e$ of $M / c \backslash \ell$. Then $e$ is a point of $M$ and, by (iii), $r_{M / c}(\{e, \ell\})=r_{M / c}(\{e\})=1$, so

$$
r_{M}(\{c, e, \ell\})=r_{M}(\{c, e\})=1+r(\{c\}) .
$$

Suppose $c$ is a point. If $\ell$ is a line, then $c$ and $e$ are on $\ell$, so $M \backslash e$ is 3-connected. Since $M / c$ has $e$ and $\ell$ as parallel points, $M \backslash e$ is 3 -connected having a c-minor isomorphic to $N$, a contradiction. Thus we may assume that $\ell$ is a point. Then $\{e, \ell, c\}$ is a triangle in $M$. Thus, for $\{c, d\}=\{a, b\}$, we see that $(X \cup \ell \cup c,\{d\})$ is a 2-separation of $M$ unless $d$ is a point of $M$. In the exceptional case, $M$ has $e, c, \ell, d$ as a fan with $M / c$ having a c-minor isomorphic to $N$. Thus, by Lemmas 43 and 58, we have a contradiction.

We may now assume that $c$ is a line. Then $r(\{c, e\})=3$, so, by $(18), r(\{c, \ell\})=3$. Thus $(X,\{c, \ell\})$ is a 2-separation of $M \backslash d$ where $\{c, d\}=\{a, b\}$. Moreover, by hypothesis, $d$ is a point. Thus, by Lemma 40 , we obtain the contradiction that $M / d$ is 3 -connected unless $M / d$ has a parallel pair $\left\{z_{1}, z_{2}\right\}$ of points. In the exceptional case, we deduce that $z_{1}$, say, is $\ell$. Hence $(X \cup \ell \cup d,\{c\})$ is a 2-separation of $M$, a contradiction. We conclude that (v) holds.

To prove (vi), assume that $\left(Y_{a}-\ell\right) \cap\left(Y_{b}-\ell\right)=\emptyset$. Then $Y_{b}-\ell \subseteq X_{a} \cup a$. But $\ell \in \operatorname{cl}\left(\left(Y_{b}-\ell\right) \cup b\right)$ and $b \in X_{a}$, so $\ell \in \operatorname{cl}\left(X_{a} \cup a\right)$. Because $M$ is 3-connected, it follows that $Y_{a}-\ell$ consists of a single point $a^{\prime}$. By symmetry, $Y_{b}-\ell$ consists of a single point $b^{\prime}$. Then $\left(X_{a} \cup a, Y_{a}-\ell\right)$ is not a 2-separation of $M \backslash \ell$, so, by (v), each of $a$ and $b$ is a line of $M$.

To finish the proof of (vi), it remains to show that, when $r(\{a, b\})=4$, the element $\ell$ is a point of $M$. Assume $\ell$ is a line. Then, in $M / a$, we have $a^{\prime}$ and $\ell$ as parallel points, so $M / a \backslash a^{\prime}$, and hence $M \backslash a^{\prime}$, has a c-minor isomorphic to $N$. As $\ell \in \operatorname{cl}_{M / a}\left(\left\{a^{\prime}\right\}\right)$, it follows that $\ell \in \operatorname{cl}_{M}(X \cup a)$. By symmetry, $\ell \in \mathrm{cl}_{M}(X \cup b)$. Thus

$$
\begin{aligned}
r(X)+2+r(X)+2 & =r(X \cup a)+r(X \cup b) \\
& =r(X \cup a \cup \ell)+r(X \cup b \cup \ell) \\
& \geqslant r(X \cup \ell)+r(M) \\
& =r(X \cup \ell)+r(X)+3 .
\end{aligned}
$$


Thus

$$
r(X \cup \ell) \leqslant r(X)+1
$$

Then

$$
\begin{aligned}
3+r(X)+1 & \geqslant r\left(\left\{a^{\prime}, a, \ell\right\}\right)+r(X \cup \ell) \\
& \geqslant r\left(\left\{a^{\prime}, \ell\right\}\right)+r\left(X \cup\left\{a^{\prime}, a, \ell\right\}\right) \\
& =r\left(\left\{a^{\prime}, \ell\right\}\right)+r(X \cup a) \\
& =r\left(\left\{a^{\prime}, \ell\right\}\right)+r(X)+2 .
\end{aligned}
$$

We deduce that $r\left(\left\{a^{\prime}, \ell\right\}\right)=2$, so $a^{\prime}$ is a point on the line $\ell$. Thus $M \backslash a^{\prime}$ is 3 -connected having a c-minor isomorphic to $N$, a contradiction.

Next we eliminate the possibility that $M_{Y}$ is $P_{1}$.

Lemma 73. $M_{Y}$ is not isomorphic to $P_{1}$.

Proof. Assume $M_{Y}$ is isomorphic to $P_{1}$. Since $\{a, b\}$ is a series pair in $M \backslash \ell$, it follows that both $M / a$ and $M / b$ have c-minors isomorphic to $N$. Hence neither $M / a$ nor $M / b$ is 3-connected.

We show next that

73.1. $\ell$ is a line of $M$.

Assume $\ell$ is a point. Then $\{\ell, a, b\}$ is a triad of $M$. Since neither $M / a$ nor $M / b$ is 3 -connected, it follows by Lemma 42 that $M$ has a triangle containing $a$ and exactly one of $b$ and $\ell$. If $M$ has $\{a, b, c\}$ as a triangle, then $M / a$ has $\{b, c\}$ as a parallel pair of points. Thus $M / a \backslash b$, and hence $M \backslash b$, has a c-minor isomorphic to $N$. Thus $b$ is a doubly labelled point, a contradiction to Lemma 58. We deduce that $M$ has $\{a, \ell\}$ in a triangle with a point $d$, say. Then $M$ has $d, a, \ell, b$ as a fan with $M / a$ having a c-minor isomorphic to $N$. Thus, by Lemmas 43 and 58, we have a contradiction. We conclude that 73.1 holds.

By Lemma $72, M / a$ and $M / b$ have 2-separations $\left(X_{a}, Y_{a}\right)$ and $\left(X_{b}, Y_{b}\right)$ such that $\ell \in Y_{a} \cap Y_{b}$. Moreover, both $M / a$ and $M / b$ have special $N$-minors, and

(i) $b \in X_{a}$ and $a \in X_{b}$;

(ii) both $Y_{a}$ and $Y_{b}$ properly contain $\{\ell\}$;

(iii) $\left(X_{a}, Y_{a}-\ell\right)$ and $\left(X_{b}, Y_{b}-\ell\right)$ are 2-separating partitions of $M / a \backslash \ell$ and $M / b \backslash \ell$, respectively, and $\ell \in \operatorname{cl}_{M / a}\left(Y_{a}-\ell\right)$ and $\ell \in \operatorname{cl}_{M / b}\left(Y_{b}-\ell\right)$;

(iv) $\left(X_{a} \cup a, Y_{a}-\ell\right)$ and $\left(X_{b} \cup b, Y_{b}-\ell\right)$ are 2-separating partitions of $M \backslash \ell$; and

(v) $\left(Y_{a}-\ell\right) \cap\left(Y_{b}-\ell\right) \neq \emptyset$.

73.2. $\left(Y_{a}-\ell\right) \cup\left(Y_{b}-\ell\right)=E-\{a, b, \ell\}$. 
We know that $\lambda_{M \backslash \ell}\left(Y_{a}-\ell\right)=1=\lambda_{M \backslash \ell}\left(Y_{b}-\ell\right)$ and $\left(Y_{a}-\ell\right) \cap\left(Y_{b}-\ell\right) \neq \emptyset$, so $\lambda_{M \backslash \ell}\left(\left(Y_{a}-\ell\right) \cap\left(Y_{b}-\ell\right)\right) \geqslant 1$. Thus, by applying the submodularity of the connectivity function, we see that

$$
\begin{aligned}
1+1 & =\lambda_{M \backslash \ell}\left(Y_{a}-\ell\right)+\lambda_{M \backslash \ell}\left(Y_{b}-\ell\right) \\
& \geqslant \lambda_{M \backslash \ell}\left(\left(Y_{a}-\ell\right) \cap\left(Y_{b}-\ell\right)\right)+\lambda_{M \backslash \ell}\left(\left(Y_{a}-\ell\right) \cup\left(Y_{b}-\ell\right)\right) \\
& \geqslant 1+\lambda_{M \backslash \ell}\left(\left(Y_{a}-\ell\right) \cup\left(Y_{b}-\ell\right)\right) .
\end{aligned}
$$

Since $M \backslash \ell$ is 2-connected, we deduce that $\lambda_{M \backslash \ell}\left(\left(Y_{a}-\ell\right) \cup\left(Y_{b}-\ell\right)\right)=1$.

This application of the submodularity of the connectivity function is an example of an 'uncrossing' argument. For the rest of the paper, we will omit the details of such arguments and will follow the practice of using the abbreviation by uncrossing to mean 'by applying the submodularity of the connectivity function.'

Now $\left(X_{a} \cup a, Y_{a}\right)$ is not a 2-separation of $M$, so, as $\ell \in \mathrm{cl}_{M / a}\left(Y_{a}-\ell\right)$, we see that

$$
r\left(Y_{a}-\ell\right)<r\left(Y_{a}\right) \leqslant r\left(Y_{a} \cup a\right)=r\left(\left(Y_{a}-\ell\right) \cup a\right) \leqslant r\left(Y_{a}-\ell\right)+1 .
$$

Hence

$$
r\left(Y_{a}\right)=r\left(Y_{a} \cup a\right)=r\left(\left(Y_{a}-\ell\right) \cup a\right)=r\left(Y_{a}-\ell\right)+1 .
$$

Thus $r\left(\left(Y_{a}-\ell\right) \cup\left(Y_{b}-\ell\right) \cup\{a, b\}\right)=r\left(Y_{a} \cup Y_{b} \cup\{a, b\}\right)=r\left(Y_{a} \cup Y_{b}\right) \leqslant r\left(\left(Y_{a}-\ell\right) \cup\left(Y_{b}-\ell\right)\right)+1$. Also, as $\{a, b\}$ is a series pair of points in $M \backslash \ell$, we see that $r\left(X_{a} \cap X_{b}\right) \leqslant r\left(\left(X_{a} \cap X_{b}\right) \cup\right.$ $\{a, b\})-1$. Therefore, $\lambda_{M}\left(Y_{a} \cup Y_{b} \cup\{a, b\}\right) \leqslant 1$. Thus, we may assume that $X_{a} \cap X_{b}$ consists of a single point, $z$, otherwise $\left(Y_{a}-\ell\right) \cup\left(Y_{b}-\ell\right)=E-\{a, b, \ell\}$ as desired.

Now $\lambda_{M \backslash \ell}\left(X_{a} \cap X_{b}\right)=\lambda_{M \backslash \ell}(\{a, b, z\})=1$. If $a \notin \operatorname{cl}(\{b, z\})$, then $\lambda_{M \backslash \ell}\left(\left(Y_{a}-\ell\right) \cup\left(Y_{b}-\right.\right.$ $\ell) \cup a) \leqslant 1$, so $\lambda_{M}\left(\left(Y_{a}-\ell\right) \cup\left(Y_{b}-\ell\right) \cup a \cup \ell\right)=\lambda_{M}\left(Y_{a} \cup Y_{b} \cup a\right) \leqslant 1$, a contradiction. Thus $a \in \operatorname{cl}(\{b, z\})$. Hence $\{a, b, z\}$ is a triangle of $M$. It follows that the point $b$ is doubly labelled, a contradiction to Lemma 58. We conclude that 73.2 holds.

73.3. $Y_{a}-Y_{b} \neq \emptyset$ and $Y_{b}-Y_{a} \neq \emptyset$.

By symmetry, it suffices to prove the first of these. Assume $Y_{a}-Y_{b}=\emptyset$. Then, as $\left(Y_{a}-\ell\right) \cup\left(Y_{b}-\ell\right)=E-\{a, b, \ell\}$, we deduce that $X_{b}=\{a\}$, so $\left(X_{b}, Y_{b}-\ell\right)$ is not a 2-separation of $M \backslash \ell / b$, a contradiction. Thus 73.3 holds.

By 73.3 and the fact that $\left(X_{a} \cup a\right) \cap\left(X_{b} \cup b\right)$ contains $\{a, b\}$, we see that each of $X_{a} \cup a$ and $X_{b} \cup b$ has at least three elements. It follows by the definition of $\mu(\ell)$ that each of $Y_{a}-\ell$ and $Y_{b}-\ell$ has exactly two elements. Since each of $Y_{a}-Y_{b},\left(Y_{a} \cap Y_{b}\right)-\ell$, and $Y_{b}-Y_{a}$ is non-empty, each of these sets has exactly one element. As the union of these sets is $E-\{\ell, a, b\}$, we deduce that $|E(M)|=6$ and $\left|X_{a} \cup a\right|=3$. Since at least one of $a$ and $b$ is not in $E(N)$, we deduce that each of $X_{a} \cup a$ and $Y_{a}-\ell$ contains at most two elements of $N$, a contradiction as one of these sets must contain at least three elements of $E(N)$. We conclude that Lemma 73 holds.

Lemma 74. $M_{Y}$ is not isomorphic to $P_{7}$.

Proof. Assume that $M_{Y}$ is isomorphic to $P_{7}$, letting $a$ be the line. Then, by Lemma 72 , $M / a$ and $M / b$ have 2-separations $\left(X_{a}, Y_{a}\right)$ and $\left(X_{b}, Y_{b}\right)$ such that $\ell \in Y_{a} \cap Y_{b}$. Moreover, both $M / a$ and $M / b$ have special $N$-minors, and 
(i) $b \in X_{a}$ and $a \in X_{b}$;

(ii) both $Y_{a}$ and $Y_{b}$ properly contain $\{\ell\}$;

(iii) $\left(X_{a}, Y_{a}-\ell\right)$ and $\left(X_{b}, Y_{b}-\ell\right)$ are 2-separating partitions of $M / a \backslash \ell$ and $M / b \backslash \ell$, respectively, and $\ell \in \mathrm{cl}_{M / a}\left(Y_{a}-\ell\right)$ and $\ell \in \mathrm{cl}_{M / b}\left(Y_{b}-\ell\right)$;

(iv) $\left(X_{a} \cup a, Y_{a}-\ell\right)$ and $\left(X_{b} \cup b, Y_{b}-\ell\right)$ are 2-separating partitions of $M \backslash \ell$; and

(v) $\left(Y_{a}-\ell\right) \cap\left(Y_{b}-\ell\right) \neq \emptyset$.

We show next that

74.1. $X_{a} \cap X_{b}$ is empty or consists of a single point.

Suppose $b \notin \operatorname{cl}\left(Y_{b}\right)$. Then $r\left(Y_{b} \cup b\right)=r\left(Y_{b}\right)+1$. Thus $\left(X_{b} \cup b, Y_{b}\right)$ is a 2-separation of $M$, a contradiction. Hence $r\left(Y_{b} \cup b\right)=r\left(Y_{b}\right)$. Now

$$
\begin{aligned}
r\left(\left(Y_{a}-\ell\right) \cup\left(Y_{b}-\ell\right)\right)+2 & \geqslant r\left(\left(Y_{a}-\ell\right) \cup\left(Y_{b}-\ell\right) \cup a\right) \\
& =r\left(Y_{a} \cup\left(Y_{b}-\ell\right) \cup a\right) \\
& =r\left(Y_{a} \cup Y_{b} \cup a\right) \\
& =r\left(Y_{a} \cup Y_{b} \cup a \cup b\right) .
\end{aligned}
$$

Also $r\left(X_{a} \cap X_{b}\right) \leqslant r\left(\left(X_{a} \cup a\right) \cap\left(X_{b} \cup b\right)\right)-2$ since $X_{a} \cap X_{b} \subseteq X$ and $\sqcap_{M}(X, Y)=1$ while $r_{M}(\{a, b\})=3$. Thus

$$
\begin{aligned}
\lambda_{M}\left(X_{a} \cap X_{b}\right) & =r\left(Y_{a} \cup Y_{b} \cup a \cup b\right)+r\left(X_{a} \cap X_{b}\right)-r(M) \\
& \leqslant r\left(\left(Y_{a}-\ell\right) \cup\left(Y_{b}-\ell\right)\right)+2+r\left(\left(X_{a} \cup a\right) \cap\left(X_{b} \cup b\right)\right)-2-r(M \backslash \ell) \\
& =\lambda_{M \backslash \ell}\left(\left(X_{a} \cup a\right) \cap\left(X_{b} \cup b\right)\right) \\
& =1,
\end{aligned}
$$

where the second-last step follows by uncrossing $\left(X_{a} \cup a, Y_{a}-\ell\right)$ and $\left(X_{b} \cup b, Y_{b}-\ell\right)$. We deduce that 74.1 holds.

74.2. $E(M)-\{\ell, a, b\}$ contains no point $\gamma$ such that $\{a, b, \gamma\}$ is 2-separating in $M \backslash \ell$.

To see this, suppose that such a point $\gamma$ exists. Recall that $M \backslash \ell$ has $N$ as a c-minor so at most one element of $\{a, b\}$ is in $E(N)$. Thus at most two elements of $\{a, b, \gamma\}$ are in $E(N)$. But $|E(N)| \geqslant 4$. Hence $\{a, b, \gamma\}$ is the non- $N$-side of a 2-separation of $M \backslash \ell$ contradicting the fact that $\mu(\ell)=2$. We conclude that 74.2 holds.

An immediate consequence of 74.2 is that $X_{a} \cap X_{b}$ does not consist of a single point. Hence, by $74.1, X_{a} \cap X_{b}=\emptyset$. As $\left(X_{a}, Y_{a}\right)$ is a 2-separation of $M / a$, it follows that $X_{a}$ cannot contain just the element $b$. Thus $\left(X_{a} \cup a\right) \cap\left(Y_{b}-\ell\right) \neq \emptyset$. We show next that

74.3. $\left(X_{b} \cup b\right) \cap\left(Y_{a}-\ell\right) \neq \emptyset$.

Suppose $\left(X_{b} \cup b\right) \cap\left(Y_{a}-\ell\right)=\emptyset$. Then $Y_{b}-\ell=E(M)-\{a, b, \ell\}=X$ so $r\left(Y_{b}-\ell\right)=$ $r(M)-2$. Hence $r\left(\left(Y_{b}-\ell\right) \cup b\right) \leqslant r(M)-1$. But $\ell \in \operatorname{cl}_{M / b}\left(Y_{b}-\ell\right)$. Thus $r\left(Y_{b} \cup b\right) \leqslant r(M)-1$, so $\{a\}$ is 2 -separating in $M$, a contradiction. We deduce that 74.3 holds. 
By uncrossing, $\lambda_{M \backslash \ell}\left(\left(X_{b} \cup b\right) \cap\left(Y_{a}-\ell\right)\right)=1=\lambda_{M \backslash \ell}\left(\left(X_{a} \cup a\right) \cap\left(Y_{b}-\ell\right)\right)$. As $\ell$ is in both $\operatorname{cl}\left(\left(Y_{a}-\ell\right) \cup a\right)$ and $\operatorname{cl}\left(\left(Y_{b}-\ell\right) \cup b\right)$, we deduce that each of $\left(X_{a} \cup a\right) \cap\left(Y_{b}-\ell\right)$ and $\left(X_{b} \cup b\right) \cap\left(Y_{a}-\ell\right)$ consists of a single point. Thus we get a contradiction to 74.2 that completes the proof of Lemma 74.

On combining Lemmas 70, 73, and 74, we immediately obtain the following.

Corollary 75. The non-N-side of every 2-separation of $M \backslash \ell$ does not contain any points.

Lemma 76. $M_{Y}$ is not isomorphic to $P_{9}$.

Proof. Assume $M_{Y}$ is isomorphic to $P_{9}$. Since each of $M_{Y} \backslash \ell / a$ and $M_{Y} \backslash \ell / b$ consists of a line through $p$, it follows that both $M / a$ and $M / b$ have c-minors isomorphic to $N$. Hence neither $M / a$ nor $M / b$ is 3-connected. Then $M / a$ and $M / b$ have 2-separations $\left(X_{a}, Y_{a}\right)$ and $\left(X_{b}, Y_{b}\right)$ such that $\ell \in Y_{a} \cap Y_{b}$. Moreover, by Lemma 72 ,

(i) $b \in X_{a}$ and $a \in X_{b}$;

(ii) both $Y_{a}$ and $Y_{b}$ properly contain $\{\ell\}$;

(iii) $\left(X_{a}, Y_{a}-\ell\right)$ and $\left(X_{b}, Y_{b}-\ell\right)$ are 2-separating partitions of $M / a \backslash \ell$ and $M / b \backslash \ell$, respectively, and $\ell \in \mathrm{cl}_{M / a}\left(Y_{a}-\ell\right)$ and $\ell \in \mathrm{cl}_{M / b}\left(Y_{b}-\ell\right)$;

(iv) $\left(X_{a} \cup a, Y_{a}-\ell\right)$ and $\left(X_{b} \cup b, Y_{b}-\ell\right)$ are 2-separating partitions of $M \backslash \ell$; and

(v) either $\left(Y_{a}-\ell\right) \cap\left(Y_{b}-\ell\right) \neq \emptyset$; or each of $X_{b} \cap\left(Y_{a}-\ell\right)$ and $X_{a} \cap\left(Y_{b}-\ell\right)$ consists of a single point, both $a$ and $b$ are lines of $M$, and $\ell$ is a point of $M$.

76.1. $\left(Y_{a}-\ell\right) \cap\left(Y_{b}-\ell\right) \neq \emptyset$.

Assume the contrary. Then, by $(\mathrm{v}), X_{b} \cap\left(Y_{a}-\ell\right)$ consists of a point, $a^{\prime}$, say. By (iii), $\ell \in \operatorname{cl}_{M / a}\left(\left\{a^{\prime}\right\}\right)$, so $\ell \in \operatorname{cl}\left(\left\{a^{\prime}, a\right\}\right)$. As $r(M)-3=r(X)$, it follows that $r(X \cup a \cup \ell) \leqslant$ $r(M)-1$. Hence the line $\{b\}$ is 2-separating in $M$, a contradiction. Thus 76.1 holds.

76.2. $\left|\left(Y_{a}-\ell\right) \cup\left(Y_{b}-\ell\right)\right| \geqslant 2$.

Assume $\left(Y_{a}-\ell\right) \cup\left(Y_{b}-\ell\right)$ contains a unique element, $z$. Then, by 76.1, $z \in\left(Y_{a}-\ell\right) \cap$ $\left(Y_{b}-\ell\right)$. Now $\ell \in \mathrm{cl}_{M / a}(\{z\})$, so $\ell \in \mathrm{cl}_{M}(\{z, a\})$. Thus

$$
r(X \cup a \cup \ell)=r(X \cup a)=r(X)+2=r(M)-1,
$$

$\operatorname{so}(X \cup a \cup \ell,\{b\})$ is a 2-separation of $M$, a contradiction. Thus 76.2 holds.

By 76.1 and uncrossing, we see that $\lambda_{M \backslash \ell}\left(\left(X_{a} \cup a\right) \cap\left(X_{b} \cup b\right)\right)=1$. Next we show the following.

76.3. $\left(Y_{a}-\ell\right) \cup\left(Y_{b}-\ell\right)$ is the non- $N$-side of a 2 -separation of $M \backslash \ell$ and it is a 2 -element set, both members of which are lines. 
By 76.2, $\left(\left(X_{a} \cup a\right) \cap\left(X_{b} \cup b\right),\left(Y_{a}-\ell\right) \cup\left(Y_{b}-\ell\right)\right)$ is a 2-separation of $M \backslash \ell$. Suppose $\left(X_{a} \cup a\right) \cap\left(X_{b} \cup b\right)$ is the non- $N$-side of this 2-separation. Then, as $\mu(\ell)=2$, we deduce that $\left(X_{a} \cup a\right) \cap\left(X_{b} \cup b\right)=\{a, b\}$. Thus, as $\ell \in \operatorname{cl}\left(\left(Y_{a}-\ell\right) \cup a\right)$,

$$
\begin{aligned}
r(M)+1 & =r\left(\left(Y_{a}-\ell\right) \cup\left(Y_{b}-\ell\right)\right)+r(\{a, b\}) \\
& =r\left(\left(Y_{a}-\ell\right) \cup\left(Y_{b}-\ell\right) \cup a\right)+r(\{b\}) \\
& =r\left(Y_{a} \cup Y_{b} \cup a\right)+r(\{b\}) .
\end{aligned}
$$

Hence $\{b\}$ is 2 -separating in $M$, a contradiction. Thus $\left(Y_{a}-\ell\right) \cup\left(Y_{b}-\ell\right)$ must be the non- $N$ side of a 2-separation of $M \backslash \ell$, so this set has cardinality two. Moreover, by Corollary 75 , both elements of this set are lines. Thus 76.3 holds.

We deduce from 76.3 that $Y_{a}-\ell$ and $Y_{b}-\ell$ are the non- $N$-sides of 2 -separations of $M \backslash \ell$. Thus, by symmetry, we may assume that $Y_{b}-\ell \subseteq Y_{a}-\ell$. Hence

$$
\left(Y_{a}-\ell\right) \cup\left(Y_{b}-\ell\right)=Y_{a}-\ell .
$$

76.4. $\left(Y_{a} \cup\{a, b\}, X_{a} \cap X_{b}\right)$ is a 2-separation of $M$.

Since $Y_{a}-\ell \supseteq Y_{b}-\ell$, we have

$$
r\left(Y_{a} \cup a\right)=r\left(\left(Y_{a}-\ell\right) \cup a\right)=r\left(Y_{a}-\ell\right)+2
$$

and

$$
r\left(Y_{a} \cup b\right)=r\left(\left(Y_{a}-\ell\right) \cup b\right)=r\left(Y_{a}-\ell\right)+2 .
$$

Moreover,

$$
r\left(Y_{a} \cup\{a, b\}\right)=r\left(\left(Y_{a}-\ell\right) \cup\{a, b\}\right) \geqslant r\left(Y_{a}-\ell\right)+3 .
$$

Thus, by submodularity,

$$
\begin{aligned}
r\left(Y_{a}-\ell\right)+2+r\left(Y_{a}-\ell\right)+2 & =r\left(Y_{a} \cup a\right)+r\left(Y_{a} \cup b\right) \\
& \geqslant r\left(Y_{a} \cup a \cup b\right)+r\left(Y_{a}\right) \\
& \geqslant r\left(Y_{a}-\ell\right)+3+r\left(Y_{a}\right) \\
& \geqslant r\left(Y_{a}-\ell\right)+3+r\left(Y_{a}-\ell\right)+1,
\end{aligned}
$$

where the last step follows because $\ell \notin \operatorname{cl}\left(Y_{a}-\ell\right)$.

We see that equality must hold throughout the last chain of inequalities. Hence $r\left(Y_{a}\right)=r\left(Y_{a}-\ell\right)+1$ and $r\left(Y_{a} \cup\{a, b\}\right)=r\left(Y_{a}-\ell\right)+3=r\left(Y_{a}\right)+2$. As $\lambda_{M \backslash \ell}\left(Y_{a}-\ell\right)=1$, it follows that $\lambda_{M}\left(Y_{a}\right)=2$, that is,

$$
r\left(Y_{a}\right)+r\left(\left(X_{a} \cup a\right) \cap\left(X_{b} \cup b\right)\right)-r(M)=2 .
$$

Hence

$$
\begin{array}{rrr}
r\left(Y_{a} \cup\{a, b\}\right)+r\left(X_{a} \cap X_{b}\right)-r(M) & \leqslant r\left(Y_{a}\right)+2+r\left(\left(X_{a} \cup a\right) \cap\left(X_{b} \cup b\right)\right) \\
& =1 . & -3-r(M)
\end{array}
$$


Thus $\left(Y_{a} \cup\{a, b\}, X_{a} \cap X_{b}\right)$ is a 2-separating partition of $M$. Since $\left(X_{a} \cup a\right) \cap\left(X_{b} \cup b\right)$ is the $N$-side of a 2-separation of $M \backslash \ell$, it follows that $X_{a} \cap X_{b}$ contains at least two elements of $E(N)$ as $\{a, b\}$ contains at most one element of $E(N)$. Thus $\left(Y_{a} \cup\{a, b\}, X_{a} \cap X_{b}\right)$ is a 2-separation of $M$, that is, 76.4 holds. But 76.4 gives a contradiction and thereby completes the proof of Lemma 76.

We now know that there are only three possibilities for $M_{Y}$, namely $P_{5}, P_{6}$, or $P_{8}$. The next few lemmas will be useful in treating all three cases.

Lemma 77. Assume $M \backslash \ell$ has $(X,\{a, b\})$ as a 2-separation where $r(\{a, b\})=3$ and each of $a$ and $b$ is a line. Then $r(X \cup \ell)=r(X)+1$ if and only if $\{a, b\}$ is a prickly 3-separating set in $M$.

Proof. If $\{a, b\}$ is a 3 -separating set in $M$, then $r(X \cup \ell)=r(M)-1$. But $r(X)=r(M)-2$, so $r(X \cup \ell)=r(X)+1$. Conversely, if $r(X \cup \ell)=r(X)+1$, then $r(X \cup \ell)=r(M)-1$, so $\{a, b\}$ is a 3-separating set in $M$. Now $r(X \cup \ell \cup a)=r(M)$ otherwise $\{b\}$ is 2-separating in $M$. By symmetry, $r(X \cup \ell \cup b)=r(M)$. Hence $\{a, b\}$ is a prickly 3-separating set in $M$.

Lemma 78. Assume $M$ has $\{a, b\}$ as a prickly 3-separating set that is 2-separating in $M \backslash \ell$. Then $M \downarrow a$ and $M \downarrow b$ are 3-connected having c-minors isomorphic to $N$.

Proof. By Lemma 54, $M \downarrow a$ and $M \downarrow b$ are 3-connected. Since $M_{X}$ and $M_{Y}$ have ground sets $X \cup p$ and $\{a, b, p\}$, we see that $r\left(M_{Y}\right)=3$. By Lemma 18, $M \downarrow a \backslash \ell=M \backslash \ell \downarrow a$. But $M \backslash \ell \downarrow a$ equals the 2 -sum of $M_{X}$ and the 2-polymatroid consisting of a line $b$ through the point $p$. Compactifying $b$ in $M \backslash \ell \downarrow a$ gives the 2-polymatroid that is obtained from $M_{X}$ by relabelling $p$ by $b$. Hence $M \downarrow a \| \ell$ has a c-minor isomorphic to $N$. Thus, using symmetry, so do $M \downarrow a$ and $M \downarrow b$.

Lemma 79. If $M_{Y}$ is $P_{5}, P_{6}$, or $P_{8}$, then $r(X \cup \ell)=r(X)+2$, so $\ell$ is a line.

Proof. Assume $r(X \cup \ell)=r(X)+1$. Then, by Lemma 77, \{a,b\} is a prickly 3-separating set in $M$. Then, by Lemma 78, $M \downarrow a$ and $M \downarrow b$ are 3 -connected having c-minors isomorphic to $N$, a contradiction to the fact that $(M, N)$ is a counterexample to Theorem 57 . Thus $r(X \cup \ell) \neq r(X)+1$. Since $\ell \notin \operatorname{cl}(X)$, we deduce that $r(X \cup \ell)=r(X)+2$, so $\ell$ is a line.

Next we deal with the case when $M \backslash \ell$ has $(X, Y)$ as its only 2-separation with $|Y|=2$, beginning with the possibility that $M_{Y}=P_{6}$.

Lemma 80. Suppose $M_{Y}=P_{6}$ and $(X, Y)$ is the only non-trivial 2-separation of $M \backslash \ell$. Then

(i) $M \rrbracket a$ or $M \rrbracket b$ is 3-connected having a special $N$-minor; or

(ii) each of $\{a, \ell\}$ and $\{b, \ell\}$ is a prickly 3-separator of $M$, and each of $M \downarrow a$ and $M \downarrow b$ is 3-connected having a c-minor isomorphic to $N$. 
Proof. By Lemma 79, $\ell$ is a line of $M$ and $\sqcap(X, \ell)=0$. In $M \backslash \ell$, we see that $a$ and $b$ are parallel points. Hence each of $M \backslash a$ or $M \backslash b$ has a special $N$-minor. But $r(E-\{a, b, \ell\})=$ $r(M)-2$ and $r(E-\{a, \ell\})=r(M)-1$, so $\{\ell\}$ is 2-separating in $M \backslash a$. Now both $M \backslash a$ or $M \backslash b$ have special $N$-minors. Hence we may assume that neither of these polymatroids is 3 -connected.

Next we show that

80.1. $r(\{a, \ell\})=3=r(\{b, \ell\})$.

We shall show that $r(\{b, \ell\})=3$, which, by symmetry, will suffice. As $M \rrbracket a$ is not 3-connected, $M \backslash a$ has a non-trivial 2-separation $(A, B)$ in which $A$ contains $\ell$. Then $(A-\ell, B)$ is a 2-separating partition of $M \backslash a \backslash \ell$. Observe that $r(M \backslash a \backslash \ell)=r(M)-1$. Suppose $b \in B$. Then $r(B \cup a)=r(B)+1$. Thus $(A-\ell, B \cup a)$ is a 2-separating partition of $M \backslash \ell$. Since $B \cup a \neq\{a, b\}$, we deduce that $A-\ell$ contains a unique element. Moreover, as $\sqcap(X, \ell)=0$, it follows that $r(A)=r(A-\ell)+2$. Thus $(A-\ell, B \cup a)$ is a 1-separating partition of $M \backslash a$, a contradiction to Lemma 59.

We may now assume that $b \in A-\ell$. Then $((A-\ell) \cup a, B)$ is a non-trivial 2-separation of $M \backslash \ell$. Thus $(A-\ell) \cup a=\{a, b\}$, so $A=\{b, \ell\}$. Hence $B=X$ and $r(\{b, \ell\})=3$. Thus 80.1 holds.

As $r(X \cup a)=r(M)-1$, we deduce that $\{b, \ell\}$ is a prickly 3-separator of $M$. Now $M \backslash \ell \downarrow b$, which, by Lemma 18, equals $M \downarrow b \backslash \ell$, has a c-minor isomorphic to $N$. Hence so does $M \downarrow b$ and, by symmetry, $M \downarrow a$. Thus, by Lemma 54, part (ii) of the lemma holds.

Lemma 81. Suppose $M_{Y}$ is $P_{5}$ or $P_{8}$. Let a be an element of $Y$ for which $\sqcap(\{a\},\{p\})=0$. Then

(i) $M /$ a has a 2-separation; and

(ii) for every 2-separation $(A, B)$ of $M /$ a with $\ell$ in $A$,

(a) $b \in B$;

(b) $(A-\ell, B \cup a)$ is a 2-separation of $M \backslash \ell$ and $|B-b| \geqslant 2$;

(c) $|A-\ell| \leqslant 2$ and if $|A-\ell|=1$, then $A-\ell$ consists of a line of $M / a$;

(d) $r_{M / a}(A-\ell)=r_{M / a}(A)$; and

(e) $\sqcap(\{a, b\}, A-\ell)=0$.

Moreover, if $(X, Y)$ is the unique non-trivial 2-separation of $M \backslash \ell$, then $M /$ a has a unique 2 -separation $(A, B)$ with $\ell$ in $A$. Further, $A-\ell$ consists of a line of $M / a$.

Proof. Certainly $M \backslash \ell / a$ and hence $M / a$ has a c-minor isomorphic to $N$. By Lemma 79, $\ell$ is a line and $\sqcap(X, \ell)=0$. As the theorem fails, $M / a$ is not 3-connected, but, by Lemma 59 , it is 2-connected. Let $(A, B)$ be a 2-separation of $M / a$ with $\ell$ in $A$.

81.1. $b \in B$.

Suppose $b \in A$. Then $a$ is skew to $B$ in $M$, so $(A \cup a, B)$ is a 2-separation of $M$, a contradiction. Thus 81.1 holds. 
81.2. $M$ does not have a point $c$ such that $B=\{b, c\}$.

Assume the contrary. We have $r_{M / a}(A)+r_{M / a}(B)-r(M / a)=1$, that is, $r(A \cup a)-$ $2+r(\{a, b, c\})-r(M)=1$. But $r(A-\ell) \leqslant r(A \cup a)-2$ and $A-\ell=X-c$. Hence $r(X-c)+r(\{a, b, c\})-r(M) \leqslant 1$. Since $r(M)=r(M \backslash \ell)$, this implies that $(X-c,\{a, b, c\})$ is a 2-separation of $M \backslash \ell$ that violates the fact that $\mu(\ell)=2$.

If such a point $c$ exists, then $A \cup a \supseteq X \cup a$, so $r(A \cup a)=r(M)$. Hence $r(\{a, b, c\})=$ $3=r(\{a, b\})$, so $(X-c,\{a, b, c\})$ is a 2-separation of $M \backslash \ell$ that violates the choice of $Y$. Thus 81.2 holds.

Next we show that

81.3. $(A-\ell, B)$ is a 2-separation of $M \backslash \ell / a$.

Certainly $(A-\ell, B)$ is 2 -separating in $M \backslash \ell / a$. We need to show that $\max \{\mid A-$ $\ell \mid, r(A-\ell)\} \geqslant 2$. By Lemma $24, A \neq\{\ell\}$. Assume $A=\{\ell, c\}$ where $c$ is a point of $M / a$. Then $c$ is a point in $M$ as $a$ is skew to $X$. Moreover,

$$
c \in \mathrm{cl}_{M}(X-c)
$$

otherwise $(X-c,\{a, b, c\})$ is a 2-separation of $M \backslash \ell$ that violates the choice of $Y$.

By Lemma 24, $a$ is not skew to $\{c, \ell\}$, so $r_{M / a}(\{c, \ell\})<r_{M}(\{c, \ell\}) \leqslant 3$. Suppose $r_{M / a}(\{c, \ell\})=2$. Then $r_{M}(B \cup a)=r(M)-1$, so $(\{c\}, B \cup a)$ is a 1-separation of $M \backslash \ell$, a contradiction. We conclude that

81.4. $r_{M / a}(\{c, \ell\})=1$, so $r_{M}(\{a, c, \ell\})=3$ and $r(M \backslash \ell / a)=r(M / a)$.

Since $c$ and $\ell$ are parallel points in $M / a$, we deduce that $M \backslash c$ has a c-minor isomorphic to $N$. Thus $M \backslash c$ has a 2 -separation $(U, V)$ where we may assume that $\ell \in U$ and $a \in V$ otherwise $M$ has a 2-separation.

Continuing with the proof of 81.3 , next we show that

81.5. $b \in U$.

Suppose $b \in V$. Then, as $a \in V$, we see that $r(V \cup \ell) \leqslant r(V)+1$ and $r(U-\ell)=$ $r(U)-2$. Thus $U=\{\ell\}$ otherwise $(U-\ell, V \cup \ell)$ is a 1-separation of $M \backslash c$. But, by (20), $c \in \operatorname{cl}(E-c-\ell)$. Hence $(U, V \cup c)$ is a 2-separation of $M$, a contradiction. Hence 81.5 holds.

81.6. $U \neq\{\ell, b\}$.

Assume $U=\{\ell, b\}$. Then $V=(X-c) \cup a$. Thus $r(V) \geqslant r(X)+1=r(M)-1$. But $r(U) \geqslant 3$ so $(U, V)$ is not a 2-separation of $M \backslash c$. This contradiction completes the proof of 81.6 .

81.7. $M$ does not have a point $d$ such that $U=\{\ell, b, d\}$.

Assume the contrary. Then

$$
r(V)=r((X-\{c, d\}) \cup a) \geqslant r(M)-2
$$

so, as $r(U)+r(V)=r(M)+1$, we must have that

$$
r(\{\ell, b, d\})=r(U) \leqslant 3 .
$$


Thus equality must hold in each of (21) and (22).

As $r(\{a, c, \ell\})=3$, we have

$$
\begin{aligned}
r(\{b, d\})+r((X-d) \cup\{a, \ell\}) & =r(\{b, d\})+r((X-\{c, d\}) \cup\{a, \ell\}) \\
& \leqslant r(\{\ell, b, d\})+r((X-\{c, d\}) \cup a)+1 \\
& =r(M)+2 .
\end{aligned}
$$

Now $r(\{b, d\})=3$, otherwise $\{a, b, d\}$ contradicts the choice of $Y$ since at most one of $a$ and $b$ is in $E(N)$. Hence $(\{b, d\},(X-d) \cup\{a, \ell\})$ is a 3-separation of $M$. Thus $r((X-d) \cup\{a, \ell\})=r(M)-1$, so $r((X-d) \cup a) \leqslant r(M)-1$. Hence $r(X-d) \leqslant r(M)-3$, while $r(X)=r(M)-2$. Thus $(X-d,\{a, b, d\})$ is a 2-separation of $M \backslash \ell$ contradicting the choice of $Y$. We conclude that 81.7 holds.

Now recall that $\{\ell, b\} \subseteq U$ and $a \in V$. Moreover, $r(\{a, c, \ell\})=3$ and $\sqcap(a, b)=1$. Thus

$$
r(V \cup\{\ell, b\}) \leqslant r(V)+2 .
$$

Also $\ell \notin \operatorname{cl}(X \cup b)$ otherwise $\{a\}$ is 2-separating in $M$, a contradiction. Thus

$$
r(U-\{\ell, b\}) \leqslant r(U)-2 .
$$

It follows by 81.6 and 81.7 that $(U-\{\ell, b\}, V \cup\{\ell, b\})$ is a 2-separation of $M \backslash c$, so $(U-\{\ell, b\}, V \cup\{\ell, b\} \cup c)$ is a 2-separation of $M$. This contradiction completes the proof of 81.3 .

We deduce from 81.3 that (ii)(d) of the lemma holds, that is, 81.8. $r((A-\ell) \cup a)=r(A \cup a)$.

Moreover, since $a$ is skew to $X$, and $A-\ell \subseteq X$, it follows, by Lemma 24, that 81.9. $(A-\ell, B \cup a)$ is a 2-separation of $M \backslash \ell$.

Now $(A, B)$ is a 2-separation of $M / a$ and $b \in B$. Since $b$ is a point of $M / a$, it follows that $|B| \geqslant 2$, so $|B \cup a| \geqslant 3$. Hence $B \cup a$ is the $N$-side of the 2-separation $(A-\ell, B \cup a)$ of $M \backslash \ell$. At most one member of $\{a, b\}$ is in $E(N)$. Since $|E(N)| \geqslant 4$, it follows that at least two elements of $N$ are in $B-b$, so $|B-b| \geqslant 2$. Thus (ii)(b) of the lemma holds. Moreover, $|A-\ell| \leqslant 2$. Since $A-\ell$ is one side of a 2 -separation, if it contains a single element, that element is a line of $M / a$. Thus (ii)(c) of the lemma holds.

Next we observe that

81.10. $\sqcap(\{a, b\}, A-\ell)=0$.

Since $\sqcap(\{a, b\}, X)=1$, we see that $\sqcap(\{a, b\}, A-\ell) \leqslant 1$. Assume $\sqcap(\{a, b\}, A-\ell)=1$. Then $r((A-\ell) \cup\{a, b\})=r(A-\ell)+2$. But $r(A-\ell)+r(B \cup a)=r(M \backslash \ell)+1$. Thus

$$
r((A-\ell) \cup\{a, b\})+r(B-b) \leqslant r(M \backslash \ell)+1 .
$$

By 81.8, $r((A-\ell) \cup a)=r(A \cup a)$. Hence we obtain the contradiction that $(A \cup\{a, b\}, B-b)$ is a 2-separation of $M$. Thus 81.10 holds.

Now suppose that $(X, Y)$ is the unique non-trivial 2-separation of $M \backslash \ell$. We complete the proof of the lemma by showing that 
81.11. $M / a$ has a unique 2 -separation $(A, B)$ with $\ell$ in $A$. Moreover, $A-\ell$ consists of a line of $M / a$.

Let $\left(A_{1}, B_{1}\right)$ and $\left(A_{2}, B_{2}\right)$ be distinct 2-separations of $M / a$ with $\ell$ in $A_{1} \cap A_{2}$. Then $b \in B_{1} \cap B_{2}$. By (ii)(c), $\left|A_{i}-\ell\right| \leqslant 2$. Suppose $\left|A_{i}-\ell\right|=2$. Then, by (ii)(b), $\left(A_{i}-\ell, B_{i} \cup a\right)$ is a non-trivial 2-separation of $M \backslash \ell$, so $A_{i}-\ell=Y$, a contradiction as $a \notin A_{i}-\ell$. We deduce that $\left|A_{i}-\ell\right|=1$, so $A_{i}-\ell$ consists of a line $m_{i}$ of $M / a$.

Now $\left(\left\{m_{1}\right\}, B_{1} \cup a\right)$ and $\left(\left\{m_{2}\right\}, B_{2} \cup a\right)$ are 2 -separations of $M \backslash \ell$. Thus $r\left(\left\{m_{1}, m_{2}\right\}\right)=4$ otherwise one easily checks that $\left(\left\{m_{1}, m_{2}\right\},\left(B_{1} \cap B_{2}\right) \cup a\right)$ is a 2-separation of $M \backslash \ell$ that contradicts the uniqueness of $(X, Y)$. Now $\sqcap(a, X)=0$, so $\sqcap\left(a,\left\{m_{1}, m_{2}\right\}\right)=0$. Thus $r_{M / a}\left(\left\{m_{1}, m_{2}\right\}\right)=4$. But, by (ii)(d) of the lemma,

$$
\begin{aligned}
2+2 & =r_{M / a}\left(\left\{m_{1}, \ell\right\}\right)+r_{M / a}\left(\left\{m_{2}, \ell\right\}\right) \\
& \geqslant r_{M / a}\left(\left\{m_{1}, m_{2}, \ell\right\}\right)+r_{M / a}(\{\ell\}) \\
& \geqslant 4+1 .
\end{aligned}
$$

This contradiction finishes the proof of 81.11 and thereby completes the proof of the lemma.

Lemma 82. If $M_{Y}=P_{8}$, then $(X, Y)$ is not the only non-trivial 2-separation of $M \backslash \ell$.

Proof. Assume $(X, Y)$ is the unique such 2-separation. By Lemma $81, M / a$ and $M / b$ have unique 2-separations $\left(A_{1}, B_{1}\right)$ and $\left(A_{2}, B_{2}\right)$ with $\ell$ in $A_{1} \cap A_{2}$. Moreover, $A_{1}-\ell$ and $A_{2}-\ell$ consist of lines $\ell_{1}$ and $\ell_{2}$ in $M / a$ and $M / b$; and $M \backslash \ell$ has $\left(A_{1}-\ell, B_{1} \cup a\right)$ and $\left(A_{2}-\ell, B_{2} \cup b\right)$ as 2-separations.

Assume $\ell_{1} \neq \ell_{2}$. Then $\left\{b, \ell_{2}\right\} \subseteq B_{1} \cup a$, so $\ell \in \operatorname{cl}\left(B_{1} \cup a\right)$. Hence $\left(A_{1}-\ell, B_{1} \cup a \cup \ell\right)$ is a 2 -separation of $M$, a contradiction. Thus $\ell_{1}=\ell_{2}$. Hence $r\left(\left\{\ell_{1}, b, \ell\right\}\right)=r\left(\left\{\ell_{1}, b\right\}\right)=4$. But we also know that $r\left(\left\{\ell_{1}, a, \ell\right\}\right)=r\left(\left\{\ell_{1}, a\right\}\right)=4$. By Lemma 81(ii)(a) and (b), we see that $b \notin \mathrm{cl}_{M / a}\left(A_{1}\right)$, so $r\left(\left\{\ell_{1}, \ell, b, a\right\}\right) \geqslant 5$. Thus

$$
\begin{aligned}
4+4 & =r\left(\left\{\ell_{1}, \ell, b\right\}\right)+r\left(\left\{\ell_{1}, \ell, a\right\}\right) \\
& \geqslant r\left(\left\{\ell_{1}, \ell, b, a\right\}\right)+r\left(\left\{\ell_{1}, \ell\right\}\right) \\
& \geqslant 5+r\left(\left\{\ell_{1}, \ell\right\}\right) .
\end{aligned}
$$

Therefore $r\left(\left\{\ell_{1}, \ell\right\}\right) \leqslant 3$. As $\sqcap\left(\left\{\ell_{1}\right\},\{\ell\}\right)=0$, we deduce that $r(\{\ell\})=1$, a contradiction to Lemma 79.

Lemma 83. If $M_{Y}=P_{5}$, then $(X, Y)$ is not the only non-trivial 2-separation of $M \backslash \ell$.

Proof. Assume $(X, Y)$ is the unique such 2-separation. Label $Y$ so that $\sqcap(a, X)=0$ and $\sqcap(b, X)=1$.

83.1. $\sqcap(a, \ell)=0$.

Suppose $\sqcap(a, \ell)=1$. Then $r(\{a, \ell\})=3$. Now $r(E-\ell)=r(E)$ and $r(E-\{\ell, a\})=$ $r(E)-1$. Thus, by Lemma 77, $\{a, \ell\}$ is a prickly 3-separator of $M$. Now $M \backslash \ell \downarrow a$ has a c-minor isomorphic to $N$ since it is the 2-sum of $M_{X}$ and the 2-polymatroid consisting 
of the line $b$ with the point $p$ on it. But, by Lemma 18, $M \backslash \ell \downarrow a=M \downarrow a \backslash \ell$. Thus, by Lemma $54, M \downarrow a$ is 3 -connected having a c-minor isomorphic to $N$, a contradiction. We conclude that 83.1 holds.

By Lemma 81, $M / a$ has a unique 2-separation and it has the form $\left(\left\{\ell_{1}, \ell\right\}, E-\right.$ $\left.\left\{\ell_{1}, \ell, a\right\}\right)$ where $\ell_{1}$ is a line of $M / a$. Moreover, $r\left(\left\{\ell_{1}, \ell, a\right\}\right)=r\left(\left\{\ell_{1}, \ell\right\}\right)$. Now $\sqcap(\ell, a)=0$ and, by Lemma $79, \ell$ is a line of $M$. Thus

$$
r(\{a, \ell\})=4 .
$$

Now $M \backslash \ell \backslash a$, and hence $M \backslash a$, has a c-minor isomorphic to $N$. Thus $M \backslash a$ has a nontrivial 2-separation $(U, V)$. Without loss of generality, we may assume that $\ell_{1} \in U$ and $\ell \in V$ since $r\left(\left\{\ell_{1}, \ell\right\}\right)=4=r\left(\left\{\ell_{1}, \ell, a\right\}\right)$.

83.2. $b \in V$.

Suppose $b \in U$. Then, as $\sqcap(X, \ell)=0$, we see that, unless $V=\{\ell, c\}$ for some point $c$, the partition $(U \cup \ell, V-\ell)$ is a 2-separation of $M \backslash a$, so $(U \cup \ell \cup a, V-\ell)$ is a 2-separation of $M$.

Consider the exceptional case. Then $r(V-\ell)=r(V)-2=1$. Now $r(M \backslash a, \ell)=$ $r(M)-1$ and $r(U)+r(V)=r(M)+1$. We see that $r(U)=r(E-\{a, \ell, c\})=r(M)-2$. Hence $\lambda_{M \backslash a, \ell}(\{c\})=0$, a contradiction. We conclude that 83.2 holds.

We now have that $V \supseteq\{\ell, b\}$. Next observe that

83.3. $(U,(V-\ell) \cup a)$ is a 2-separation of $M \backslash \ell$, and $r((V-\ell) \cup a)=r(V)$.

To see this, first note that, since $b \in V-\ell$, we have

$$
r((V-\ell) \cup a) \leqslant r(V-\ell)+1 .
$$

We also have

$$
r(V-\ell) \leqslant r(V)-1
$$

otherwise $r(V-\ell)=r(V)$ so $\ell \in \operatorname{cl}(E-\{a, \ell\})$. But $r(E-\{a, \ell\})=r(E)-1$, so ( $E-a,\{a\})$ is a 2-separation of $M$, a contradiction. Combining (25) and (26) gives 83.3.

Since $(X, Y)$ is the unique non-trivial 2-separation of $M \backslash \ell$, we deduce that $(V-\ell) \cup a=$ $\{a, b\}$. Moreover, by 83.3, $r(\{a, b\})=3=r(\{b, \ell\})$. It follows using submodularity that $r(\{a, b, \ell\})=4$. Thus $b \in \mathrm{cl}_{M / a}(\{\ell\})$. Hence $\left(\left\{\ell_{1}, \ell, b\right\}, E-\left\{\ell_{1}, \ell, a, b\right\}\right)$ is a 2-separation of $M / a$, which contradicts the fact that $\left(\left\{\ell_{1}, \ell\right\}, E-\left\{\ell_{1}, \ell, a\right\}\right)$ is the unique 2-separation of $M / a$. This completes the proof of Lemma 83 .

By Lemma 78, $M \backslash \ell$ has no 2-element 2-separating set that is a prickly 3-separating set in $M$.

Lemma 84. Let $\{a, b\}$ and $\{c, d\}$ be disjoint 2-separating sets of $M \backslash \ell$ where each of $a$, $b, c$, and $d$ is a line, $r(\{a, b\})=3=r(\{c, d\})$, and $\sqcap(\{a\}, E-\{a, b, \ell\})=0$. Then either

(i) $M /$ a is 3-connected having a c-minor isomorphic to $N$; or

(ii) $M / \ell$ has a c-minor isomorphic to $N$ and $\ell \in \mathrm{cl}_{M / a}(\{c, d\})$. 
Proof. Assume that the lemma fails. Let $Z=E-\{\ell, a, b, c, d\}$. Then, as neither $\{a, b\}$ nor $\{c, d\}$ is a prickly 3 -separating set of $M$, by Lemma 77 , we see that

$$
\sqcap(Z \cup\{c, d\},\{\ell\})=0=\sqcap(Z \cup\{a, b\},\{\ell\}),
$$

so $\sqcap(Z,\{\ell\})=0$ and $\sqcap(\{a, b\},\{\ell\})=0$. It follows, as $\sqcap(\{a\}, Z)=0$, that

$$
\sqcap_{M / a}(Z,\{\ell\})=0 .
$$

Let $X=E-\{a, b, \ell\}$ and $Y=\{a, b\}$. Then $M \backslash \ell=M_{X} \oplus_{2} M_{Y}$ where $M_{Y}$ has ground set $\{p, a, b\}$. Then $M_{X}$ has a c-minor isomorphic to $N$. As $\sqcap(\{a\}, X)=0$, it follows that $M \backslash \ell / a$, and hence $M / a$, has a c-minor isomorphic to $N$.

84.1. $\ell \in \mathrm{cl}_{M / a}(\{c, d\})$.

Assume $\ell \notin \mathrm{cl}_{M / a}(\{c, d\})$. Since $M / a$ is not 3 -connected, it has a 2-separation $(A, B)$ with $\ell \in A$ and $b \in B$. Moreover, by Lemma 81, we know that $(A-\ell, B \cup a)$ is a 2-separation of $M \backslash \ell$, that $|B-b| \geqslant 2$, that $|A-\ell| \leqslant 2$, and that $\ell \in \operatorname{cl}_{M / a}(A-\ell)$.

Suppose $|A-\ell|=1$. Then, by Lemma 81 again, $A-\ell$ consists of a line $m$ of $M / a$ and $\ell \in \mathrm{cl}_{M / a}(\{m\})$. Thus $m \notin\{c, d\}$, so $m \in Z$ and we have a contradiction to (27). Now suppose that $|A-\ell|=2$. Then $\ell \in \operatorname{cl}_{M / a}(A-\ell)$. Thus $\{c, d\} \neq A-\ell$. If $\{c, d\}$ avoids $A-\ell$, then we again get a contradiction to (27). Thus $A-\ell$ meets $\{c, d\}$ in a single element. Then, by uncrossing the 2-separations $(A-\ell, B \cup a)$ and $(\{c, d\}, E-\{\ell, c, d\})$ of $M \backslash \ell$, we see that $(A-\ell) \cup\{c, d\})$ is a 3 -element 2-separating set in $M \backslash \ell$. At most one element of $\{c, d\}$ is in $E(N)$. Thus $(A-\ell) \cup\{c, d\}$ is the non- $N$-side of a 2-separation of $M \backslash \ell$. This is a contradiction as this set has three elements. We conclude that 84.1 holds.

We shall complete the proof of Lemma 84 by showing that $M / \ell$ has a c-minor isomorphic to $N$. In the argument that follows, it helps to think in terms of the matroids that are naturally derived from the 2-polymatroids we are considering. We know that $M \backslash \ell=M_{X} \oplus_{2} M_{Y}$ where $M_{Y}$ has ground set $\{a, b, p\}$ with $p$ being the basepoint of the 2-sum. As $\{c, d\}$ is 2-separating in $M \backslash \ell$, it is also 2-separating in $M_{X}$. Thus $M_{X}=M_{Z} \oplus_{2} M_{W}$ where $M_{W}$ has ground set $\{c, d, q\}$ with $q$ being the basepoint of this 2sum. Now $\{c, d\}$ does not span $p$ otherwise $\{a, b, c, d\}$ is 2 -separating in $M \backslash \ell$ and contains at most two elements of $N$, a contradiction to the definition of $Y$. By two applications of Lemma 51, we see that $M_{X}$, and hence $M_{Z}$, has a c-minor isomorphic to $N$.

Now $M \backslash \ell / a$ equals $M_{X}$ after relabelling the element $p$ of the latter by $b$. We will call this relabelled 2-polymatroid $M_{X}^{\prime}$. By 84.1, $M / a$ is obtained from $M_{X}^{\prime}$ by adding $\ell$ to the closure of $\{c, d\}$ as a point or a line. Thus $M / a$ is the 2 -sum with basepoint $q$ of $M_{Z}^{\prime}$ and $M_{W}^{\prime}$ where $M_{Z}^{\prime}$ is obtained from $M_{Z}$ by relabelling $p$ as $b$, while $M_{W}^{\prime}$ is obtained from $M_{W}$ by adding $\ell$. By $(27), \ell$ is skew to $Z$ in $M / a$, so $\ell$ is skew to $q$ in $M_{W}^{\prime}$. Now $\ell$ is a not a line of $M_{W}^{\prime}$, otherwise at least one of $c$ and $d$ is parallel to the basepoint $q$ in $M_{W}^{\prime}$, so $M / a / \ell$ and hence $M / \ell$ has a c-minor isomorphic to $N$. Hence $\ell$ is a point of $M_{W}^{\prime}$, so $M_{W}^{\prime} / \ell$ has rank 2. It has no point parallel to $q$ otherwise $M / a / \ell$ has a c-minor isomorphic to $N$. Thus $M_{W}^{\prime} / \ell$ can be obtained from one of $P_{1}, P_{2}$, or $P_{4}$ by relabelling the element $p$ by $q$. In the first two cases, we can contract a point from $M_{W}^{\prime} / \ell$ to obtain a 2-polymatroid consisting of two parallel points, one of which is $q$, so we get 
the contradiction that $M / a / \ell$ has a c-minor isomorphic to $N$. In the third case, deleting one of the lines, say $c$, of $M_{W}^{\prime} / \ell$ leaves $d$ as a line through $q$. Thus $\{d\}$ is 2-separating in $M / a \backslash \ell \backslash c$. Compactifying $d$, we obtain a 2-polymatroid having a c-minor isomorphic to $N$. Again we obtain the contradiction that $M / a \backslash \ell$ has a c-minor isomorphic to $N$.

Lemma 85. Let $\{a, b\}$ and $\{c, d\}$ be disjoint 2-separating sets of $M \backslash \ell$ where each of $a$, $b, c$, and $d$ is a line, $r(\{a, b\})=3=r(\{c, d\})$. Assume $M / \ell$ has a c-minor isomorphic to $N$. Then at least one of $\sqcap(\{a\}, E-\{\ell, a, b\})$ and $\sqcap(\{b\}, E-\{\ell, a, b\})$ is not equal to one.

Proof. As before, let $Z=E-\{a, b, c, d, \ell\}$. Since the theorem fails, it follows by Lemmas 59 and 78 that $M / \ell$ is 2-connected and neither $\{a, b\}$ nor $\{c, d\}$ is a prickly 3separating set of $M$. Moreover, by Lemma 79, $\ell$ is a line that is skew to each of $Z \cup\{a, b\}$ and $Z \cup\{c, d\}$. Thus, if $(R, B)$ is a 2-separation of $M / \ell$, then, by Lemma $24, \sqcap(R,\{\ell\}) \geqslant 1$ and $\sqcap(B,\{\ell\}) \geqslant 1$.

By Lemma 32,

$$
\sqcap(R,\{\ell\})+\sqcap(B,\{\ell\})+\lambda_{M / \ell}(R)=\lambda_{M \backslash \ell}(R)+\lambda_{M}(\{\ell\}),
$$

so

$$
\sqcap(R,\{\ell\})+\sqcap(B,\{\ell\})=\lambda_{M \backslash \ell}(R)+1 .
$$

As $\sqcap(\{\ell\}, Z \cup\{a, b\})=0=\sqcap(\{\ell\}, Z \cup\{c, d\})$, it follows by Lemma 25 that both $R$ and $B$ meet both $\{a, b\}$ and $\{c, d\}$. Without loss of generality, we may assume that $\{a, c\} \subseteq R$ and $\{b, d\} \subseteq B$.

Now suppose that $\sqcap(\{a\}, E-\{\ell, a, b\})=1=\sqcap(\{b\}, E-\{\ell, a, b\})$. By Lemma 31(i),

$$
\begin{aligned}
\sqcap(\{a, c\},\{b, d\})+\sqcap(\{a\},\{c\})+\sqcap(\{b\},\{d\})=\sqcap(\{a, b\},\{c, d\})+ & \sqcap(\{a\},\{b\}) \\
+ & \sqcap(\{c\},\{d\}) .
\end{aligned}
$$

As $\mu(\ell)=2$, we see that $\sqcap(\{a, b\},\{c, d\})=0$, so $\sqcap(\{a\},\{c\})=0=\sqcap(\{b\},\{d\})$. Thus

$$
\sqcap(\{a, c\},\{b, d\})=\sqcap(\{a\},\{b\})+\sqcap(\{c\},\{d\})=2 .
$$

Hence $\sqcap(R, B) \geqslant 2$, that is, $\lambda_{M \backslash \ell}(R) \geqslant 2$. Thus, by $(28), \sqcap(R,\{\ell\})=2$ or $\sqcap(B,\{\ell\})=2$. By symmetry, we may assume the former. But, as $\sqcap(\{c, d\} \cup Z,\{\ell\})=0$ and $\sqcap(\{c, d\} \cup$ $Z,\{a\})=1$, by Lemma 31(ii),

$$
\begin{aligned}
\sqcap(\{c, d\} \cup Z \cup a,\{\ell\})+1 & =\sqcap(\{c, d\} \cup Z \cup a,\{\ell\})+\sqcap(\{c, d\} \cup Z,\{a\}) \\
& =\sqcap(\{c, d\} \cup Z \cup \ell,\{a\})+\sqcap(\{c, d\} \cup Z,\{\ell\}) \\
& \leqslant 2+0 .
\end{aligned}
$$

Thus $\sqcap(\{c, d\} \cup Z \cup a,\{\ell\}) \leqslant 1$. But $R \subseteq Z \cup\{a, c\}$ so $\sqcap(R,\{\ell\}) \leqslant 1$, a contradiction.

Lemma 86. The 2-polymatroid $M \backslash \ell$ does not have two disjoint 2-element 2-separating sets. 
Proof. Assume that $M \backslash \ell$ has $\{a, b\}$ and $\{c, d\}$ as disjoint 2-separating sets. Then each of $a, b, c$, and $d$ is a line and $r(\{a, b\})=3=r(\{c, d\})$. As before, let $Z=E-\{a, b, c, d, \ell\}$. Suppose $Y$ is $\{a, b\}$ or $\{c, d\}$, and $X=E-\ell-Y$. Then $M \backslash \ell=M_{X} \oplus_{2} M_{Y}$. By Lemmas $70,71,73,74$, and 76 , we know that $M_{Y}$ is isomorphic to $P_{5}, P_{6}$, or $P_{8}$. By Lemma 79,

86.1. $\ell$ is skew to $X$, so $\ell$ is skew to each of $a, b, c$, and $d$.

When $M_{Y} \cong P_{n}$, we shall say that $Y$ is a type-n 2-separator of $M \backslash \ell$.

86.2. Neither $\{a, b\}$ nor $\{c, d\}$ is of type- 6 .

Assume the contrary. Suppose $\{a, b\}$ is of type-6. Then, by Lemma $85, M / \ell$ does not have a c-minor isomorphic to $N$. Thus, by Lemma 84, neither $\sqcap(\{c\}, X)$ nor $\sqcap(\{d\}, X)$ is 0 . Hence $\{c, d\}$ is also of type-6. Suppose $\alpha \in\{a, b\}$ and $\gamma \in\{c, d\}$. Then $r(Z \cup\{\alpha, \gamma\})=$ $r(Z)+2$. Of course, $r(M)=r(Z)+4$.

Suppose $r(Z \cup\{\alpha, \gamma\} \cup \ell)=r(M)$. Then $\sqcap(Z \cup\{\alpha, \gamma\}, \ell)=0$. Let the elements of $\{a, b, c, d\}-\{\alpha, \gamma\}$ be $\beta$ and $\delta$. In $M \backslash \beta \backslash \delta$, the set $\{\ell\}$ is 1 -separating. Thus $M \backslash \beta \backslash \delta \backslash \ell=$ $M \backslash \beta \backslash \delta / \ell$. As $M \backslash \beta \backslash \delta \backslash \ell$ has a c-minor isomorphic to $N$, so does $M / \ell$. We then get a contradiction to Lemma 85 since $\sqcap(a, E-\{\ell, a, b\})=1=\sqcap(b, E-\{\ell, a, b\})$.

We may now assume that $r(Z \cup\{\alpha, \gamma\} \cup \ell) \leqslant r(M)-1$. By 86.1, $\ell$ is skew to $Z \cup\{a, b\}$, so $r(Z \cup a \cup \ell)=r(M)-1$. Thus, using the submodularity of $r$, we have

$$
\begin{aligned}
2 r(M)-1 & =r(Z \cup a \cup \ell)+r(M) \\
& \leqslant r(Z \cup\{a, c\} \cup \ell)+r(Z \cup\{a, d\} \cup \ell) \\
& \leqslant 2 r(M)-2 .
\end{aligned}
$$

This contradiction establishes 86.2.

We now know that each of $\{a, b\}$ and $\{c, d\}$ is of type- 5 or of type- 8 . In particular, we may assume that $\sqcap(\{a\}, Z \cup\{c, d\})=0=\sqcap(\{c\}, Z \cup\{a, b\})$. Since $\mu(\ell)=2$ and $\{a, b, c, d\}$ contains at most two elements of $N$, we see that

$$
r(\{a, b, c, d\})=6 .
$$

By Lemma 84,

86.3. $\ell \in \mathrm{cl}_{M / a}(\{c, d\})$ and $\ell \in \mathrm{cl}_{M / c}(\{a, b\})$.

We deduce that $r(\{a, c, d, \ell\})=r(\{a, c, d\})=5$ and $r(\{a, b, c, \ell\})=r(\{a, b, c\})=5$. By submodularity and (29),

$$
\begin{aligned}
10 & =r(\{a, c, d, \ell\})+r(\{a, b, c, \ell\}) \\
& \geqslant r(\{a, b, c, d, \ell\})+r(\{a, c, \ell\}) \\
& \geqslant 6+4=10 .
\end{aligned}
$$

We conclude that

$$
r(\{a, c, \ell\})=4
$$

Next we show the following. 
86.4. Both $\{a, b\}$ and $\{c, d\}$ are of type-5.

Suppose $\{a, b\}$ is of type- 8 . Then $\sqcap(\{b\}, Z \cup\{c, d\})=0$. Thus we can replace $a$ by $b$ in the argument used to prove (30) to get that $r(\{b, c, \ell\})=4$. Hence

$$
\begin{aligned}
4+4 & =r(\{a, c, \ell\})+r(\{b, c, \ell\}) \\
& \geqslant r(\{a, b, c, \ell\})+r(\{c, \ell\}) \\
& \geqslant 5+4 .
\end{aligned}
$$

This contradiction and symmetry implies that 86.4 holds.

Now, by Lemma 51, $M \backslash \ell \backslash a$, and hence $M \backslash a$, has a c-minor isomorphic to $N$. Thus $M \backslash a$ is not 3-connected. Let $(U, V)$ be a non-trivial 2-separation of $M \backslash a$. Then we may assume that $\ell \in U$ and $c \in V$ otherwise $M$ has a 2-separation.

Suppose $d \in U$. Then, by 86.1, $(U \cup c, V-c)$ is a 1-separation of $M \backslash a$, a contradiction. Thus $d \in V$. By 86.1 again, $r(U-\ell)=r(U)-2$, so we obtain the contradiction that $(U-\ell, V \cup \ell \cup a)$ is a 1- or 2-separation of $M$ unless $U-\ell$ consists of a single point, $u$, and $r(U)=3$. In the exceptional case, since $M \backslash a \backslash \ell$ is 2-connected, we see that $u \in \operatorname{cl}(V)$, so $(U-u, V \cup u)$ is a 1-separation of $M \backslash a$, a contradiction.

Lemma 87. Suppose that $M$ has an element $\ell$ such that $M \backslash \ell$ has $N$ as a c-minor. Then the largest non- $N$-side in a 2-separation of $M \backslash \ell$ has size exceeding two.

Proof. Assume $\mu(\ell)=2$. Then $M \backslash \ell=M_{X} \oplus_{2} M_{Y}$ where $|Y|=2$. In Lemma 69, we identified the nine possibilities for $M_{Y}$. We showed in Lemmas 70, 71, 73, 74, and 76 that $M_{Y}$ must be isomorphic to $P_{5}, P_{6}$, or $P_{8}$. In Lemmas 80, 82, and 83, we showed that $(X, Y)$ cannot be the sole non-trivial 2-separation of $M \backslash \ell$. Lemma 86 completes the proof by showing that $M \backslash \ell$ cannot have a second non-trivial 2-separation.

Lemma 88. Suppose that $M$ has an element $\ell$ such that $M / \ell$ has $N$ as a c-minor. Then the largest non- $N$-side in a 2-separation of $M / \ell$ has size exceeding two.

Proof. By Lemma 12, $(M / \ell)^{*}$ has a c-minor isomorphic to $N^{*}$. By Lemma $2,(M / \ell)^{*}=$ $\left(M^{*} \backslash \ell\right)^{b}$. Thus $M^{*} \backslash \ell$ has a c-minor isomorphic to $N^{*}$. Let $Y$ be a largest non- $N$-side in a 2-separation of $M / \ell$. By Lemma 2 again, $Y$ is a largest non- $N^{*}$-side in a 2-separation of $M^{*} \backslash \ell$. Replacing $(M, N)$ by $\left(M^{*}, N^{*}\right)$ in Lemma 87 , we deduce that $|Y|>2$.

\section{Finding a doubly labelled line}

Recall that we are assuming that $(M, N)$ is a counterexample to Theorem 57 where $N$ is a 3-connected 2-polymatroid that is a c-minor of $M$. In this section, we prove some lemmas that will eventually enable us to deduce that $M$ has a doubly labelled line. The first step in this process is to prove the following elementary but useful lemma.

Lemma 89. Suppose $y \in E(M)-E(N)$. If $y$ is not a doubly labelled element of $M$, and $M^{\prime}$ has a special $N$-minor for some $M^{\prime}$ in $\{M \backslash y, M / y\}$, then $M^{\prime}$ has $N$ as a c-minor. 
Proof. Since $y \in E(M)-E(N)$, some $M^{\prime \prime}$ in $\{M \backslash y, M / y\}$ has $N$ as a c-minor. Since $y$ is not doubly labelled, we see that $M^{\prime \prime}=M^{\prime}$.

The next lemma identifies an important dichotomy.

Lemma 90. Let $M^{\prime}$ be a c-minor of $M$ having $N$ as a c-minor and let $\left(X^{\prime}, Y^{\prime}\right)$ be a 2-separation of $M^{\prime}$ having $X^{\prime}$ as the $N$-side. Assume that, for all elements $y$ of $Y^{\prime}$, at least one of $M^{\prime} \backslash y$ and $M^{\prime} / y$ does not have a special $N$-minor. Then either

(i) $\sqcap_{M^{\prime}}\left(\{y\}, X^{\prime}\right)=1$ for all $y$ in $Y^{\prime}$; or

(ii) $\sqcap_{M^{\prime}}\left(Y^{\prime}-y, X^{\prime}\right)=0$ for all $y$ in $Y^{\prime}$.

Proof. Suppose $y \in Y^{\prime}$. If $\sqcap_{M^{\prime}}\left(\{y\}, X^{\prime}\right)=0$, then, by Lemma 29, $\sqcap_{M^{\prime} / y}\left(X^{\prime}, Y^{\prime}-y\right)=1$, so, by Lemma 51(ii), $M^{\prime} / y$ has a special $N$-minor. If $\sqcap_{M^{\prime}}\left(Y^{\prime}-y, X^{\prime}\right)=1$, then, by Lemma 51(i), $M^{\prime} \backslash y$ has a special $N$-minor. By hypothesis, $M^{\prime} \backslash y$ or $M^{\prime} / y$ has no special $N$-minor. We deduce the following.

90.1. Either $\sqcap_{M^{\prime}}\left(\{y\}, X^{\prime}\right)=1$ or $\sqcap_{M^{\prime}}\left(Y^{\prime}-y, X^{\prime}\right)=0$.

Next we show that all the elements of $Y^{\prime}$ behave similarly.

90.2. If $\sqcap_{M^{\prime}}\left(\{y\}, X^{\prime}\right)=1$, then $\sqcap_{M^{\prime}}(\{z\}, X)=1$ for all $z$ in $Y^{\prime}$.

To see this, note first that $M^{\prime}=M_{X^{\prime}}^{\prime} \oplus_{2} M_{Y^{\prime}}^{\prime}$. Since $\sqcap_{M^{\prime}}\left(\{y\}, X^{\prime}\right)=1$, it follows that $p \in \operatorname{cl}_{M_{Y^{\prime}}^{\prime}}(\{y\})$. Suppose $z \in Y^{\prime}-y$. Then $p \in \mathrm{cl}_{M_{Y^{\prime}}^{\prime}}\left(Y^{\prime}-z\right)$. Hence $\sqcap_{M^{\prime}}\left(X^{\prime}, Y^{\prime}-z\right)=1$ so $M^{\prime} \backslash z$ has a special $N$-minor. Thus $M^{\prime} / z$ does not have a special $N$-minor. Hence, by Lemma 51(ii), $\sqcap_{M^{\prime} / z}\left(X^{\prime}, Y^{\prime}-z\right)=0$, so, by Lemma $29, \sqcap_{M^{\prime}}\left(X^{\prime},\{z\}\right)=1$, and 90.2 holds.

Now suppose that $\sqcap_{M^{\prime}}\left(\{y\}, X^{\prime}\right)=0$. Then, for all $z$ in $Y^{\prime}$, by $90.2, \sqcap_{M^{\prime}}\left(\{z\}, X^{\prime}\right)=0$ so, by $90.1, \sqcap_{M^{\prime}}\left(Y^{\prime}-z, X^{\prime}\right)=0$.

The next lemma describes what happens when (i) of Lemma 90 holds.

Lemma 91. Suppose $M \backslash \ell$ has $N$ as a c-minor. Let $(X, Y)$ be a 2-separation of $M \backslash \ell$ in which $X$ is the $N$-side and $|Y| \geqslant 3$. Then

(i) Y contains a doubly labelled element; or

(ii) $\sqcap(\{y\}, X) \neq 1$ for some $y$ in $Y$; or

(iii) $Y$ contains an element $y$ such that $M \backslash y$ has $N$ as a c-minor and every non-trivial 2-separation of $M \backslash y$ has the form $\left(Z_{1}, Z_{2}\right)$ where $Z_{1}$ is the $N$-side and $Z_{2} \subseteq Y-y$.

Proof. Suppose that $\sqcap(\{y\}, X)=1$ for all $y$ in $Y$ and that $Y$ does not contain any doubly labelled elements. As usual, we write $M \backslash \ell$ as the 2 -sum with basepoint $p$ of the 2-polymatroids $M_{X}$ and $M_{Y}$ having ground sets $X \cup p$ and $Y \cup p$, respectively. First we show that

91.1. $Y$ does not contain a point. 
Assume that $Y$ does contain a point, $z$. Then, since $\sqcap(\{z\}, X)=1$, we see that $z$ is parallel to $p$ in $M_{Y}$. By Proposition 47, $M \backslash \ell \backslash z$ is 2-connected. Hence $M \backslash z$ is 2-connected. Also, in $M_{X}$ and $M_{Y}$, the sets $X$ and $Y-z$ span $p$, and hence span $z$. We show next that 91.2. $M \backslash z$ is 3-connected.

Suppose that $M \backslash z$ has a 2-separation $(R, B)$ where $\ell \in R$. Then $(R-\ell, B)$ is 2 separating in $M \backslash z \backslash \ell$. Note that $r(M \backslash \ell)=r(M)$, so $r(M \backslash \ell \backslash z)=r(M)$. We have

$$
r(R)+r(B)=r(M \backslash z)+1 \text {. }
$$

Thus

$$
r(R-\ell)+r(B) \leqslant r(M \backslash z, \ell)+1 .
$$

Now $R \neq\{\ell\}$ otherwise $Y-z \subseteq B$ and we obtain the contradiction that $(R, B \cup z)$ is a 2-separation of $M$. Observe that, since $M \backslash \ell \backslash z$ is 2-connected, $r(R-\ell)=r(R)$. As $M$ is 3 -connected, neither $B$ nor $R-\ell$ spans $z$. Thus neither $X$ nor $Y-z$ is contained in $B$ or $R-\ell$. Hence $(X, Y-z)$ and $(R-\ell, B)$ cross.

Now $\lambda_{M \backslash \ell \backslash z}(Y-z)=\lambda_{M \backslash \ell}(Y)=1$ and $\lambda_{M \backslash \ell \backslash z}(B)=1$. Thus, by uncrossing, $\lambda_{M \backslash \ell \backslash z}(B \cap(Y-z))=1$. Since $\ell \in \operatorname{cl}(R-\ell)$ and $z \in \operatorname{cl}(X)$, we deduce that $\lambda_{M}(B \cap(Y-$ $z))=1$. As $M$ is 3-connected, it follows that $B \cap(Y-z)$ consists of a single point $y$. Then, by assumption, $\sqcap(X,\{y\})=1$. But $\sqcap(X,\{z\})=1$. Thus $y$ is parallel to $p$ in $M_{Y}$. Hence $y$ and $z$ are parallel points in $M$, a contradiction. We conclude that 91.2 holds.

To complete the proof of 91.1 , we shall show that $M \backslash z$ has a special $N$-minor. We know that $M \backslash \ell=M_{X} \oplus_{2} M_{Y}$ where $z$ is parallel in $M_{Y}$ to the basepoint $p$ of the 2-sum. Moreover, by Lemma $49, M_{X}$ has a special $N$-minor. Now $M \backslash \ell \backslash z$ is 2 -connected and, by [5, Proposition 3.1], $M \backslash \ell \backslash z=M_{X} \oplus_{2}\left(M_{Y} \backslash z\right)$. Hence $M \backslash z$ has a special $N$-minor. Thus $M \Downarrow z$ is 3 -connected having a c-minor isomorphic to $N$, a contradiction. We deduce that 91.1 holds.

We now know that every element of $Y$ is a line $y$ with $\sqcap(X,\{y\})=1$. Hence, in $M_{Y}$, the basepoint $p$ lies on $y$. Thus, for all $y$ in $Y$, we see that $M \backslash \ell \backslash y$ is 2-connected. Then, by Lemma 48 again, we deduce that

91.3. for all $y$ in $Y$, both $M \backslash \ell \backslash y$ and $M \backslash y$ have special $N$-minors.

Since every line in $Y$ contains $p$, it follows that $M_{Y} / p$ is a matroid. Next we show that 91.4. $M_{Y} / p$ has a circuit.

Assume that $M_{Y} / p$ has no circuits. Let $y$ and $y^{\prime}$ be two distinct elements of $Y$. Then $r\left(X \cup\left(Y-\left\{y, y^{\prime}\right\}\right)\right)=r(X)+\left|Y-\left\{y, y^{\prime}\right\}\right|$ and $r(X \cup Y)=r(X)+|Y|$. As a step towards 91.4, we show that

91.5. $\sqcap\left(X \cup\left(Y-\left\{y, y^{\prime}\right\}\right),\{\ell\}\right)=0$.

Suppose that $\sqcap\left(X \cup\left(Y-\left\{y, y^{\prime}\right\}\right),\{\ell\}\right) \geqslant 1$. Then, as $r(Y)=|Y|+1$,

$$
\begin{aligned}
\lambda_{M}\left(\left\{y, y^{\prime}\right\}\right) & =r\left(X \cup\left(Y-\left\{y, y^{\prime}\right\}\right) \cup \ell\right)+r\left(\left\{y, y^{\prime}\right\}\right)-r(M) \\
& \leqslant r(X)+\left|Y-\left\{y, y^{\prime}\right\}\right|+1+3-r(M) \\
& =r(X)+r(Y)-r(M \backslash \ell)+1=2 .
\end{aligned}
$$


As $M$ is 3-connected, we see that $\lambda_{M}\left(\left\{y, y^{\prime}\right\}\right)=2$, so equality holds thoughout the last chain of inequalities. Thus $\left\{y, y^{\prime}\right\}$ is a prickly 3-separator of $M$ and $\lambda_{M \backslash \ell}\left(\left\{y, y^{\prime}\right\}\right)=1$. By Lemma 54, $M \downarrow y$ is 3-connected. By Lemma 46(vi), $(M \backslash \ell) \downarrow y=M_{X} \oplus_{2}\left(M_{Y} \downarrow y\right)$. Thus $\sqcap_{M \backslash \ell \downarrow y}(X, Y-y)=1$ so, by Lemma 51(iii), $(M \downarrow y) \backslash \ell$, and hence $M \downarrow y$, has a special $N$-minor. This contradiction implies that 91.5 holds for all distinct $y$ and $y^{\prime}$ in $Y$.

As the next step towards proving 91.4, we now show that

91.6. $M / \ell$ has a c-minor isomorphic to $N$.

In $M \backslash \ell$, deleting all but one element, $y$, of $Y$ leaves the 2-polymatroid that, when $y$ is compactified, equals $M_{X}$ with $p$ relabelled as $y$. Hence $M \backslash \ell \backslash(Y-y)$ has a c-minor isomorphic to $N$. By 91.5, since $|Y| \geqslant 3$, we deduce that $\{\ell\}$ is 1-separating in $M \backslash(Y-y)$. Hence $M \backslash(Y-y) \backslash \ell=M \backslash(Y-y) / \ell$, so, by 91.3 , we deduce that 91.6 holds.

Still continuing towards the proof of 91.4 , next we observe that

91.7. $\ell$ is a line of $M$.

Suppose $\ell$ is a point. By Lemma $40, M / \ell$ is 2 -connected having one side of every 2-separation being a pair of points of $M$ that are parallel in $M / \ell$. By $91.6, M$ must have such a pair $\{u, v\}$ of points. Then both $M \backslash u$ and $M \backslash v$ have c-minors isomorphic to $N$. By Lemma 42, $M$ has a triad of points containing $\ell$ and one of $u$ and $v$, say $u$. Let $w$ be the third point in this triad. Then $M \backslash \ell$ has $\{u, w\}$ as a series pair of points, so $M \backslash \ell / u$, and hence $M / u$, has a c-minor isomorphic to $N$. Thus the point $u$ contradicts Lemma 58 .

By 91.6, $M / \ell$ has a 2-separation $(U, V)$. Thus $r(U \cup \ell)+r(V \cup \ell)-r(M)=3$. By symmetry, we may assume that $U \subseteq(X \cup Y)-\left\{y, y^{\prime}\right\}$ for some $y^{\prime}$ in $Y-y$. Then, by 91.5 and 91.7, $r(U \cup \ell)=r(U)+2$. Hence $(U, V \cup \ell)$ is a 2-separation of $M$. This contradiction completes the proof of 91.4 .

Choose $y$ in $Y$ such that $y$ is in a circuit of $M_{Y} / p$ and $y \in E(M)-E(N)$. By 91.3, $M \backslash y$ has a special $N$-minor. Thus, by Lemma $89, M \backslash y$ has $N$ as a c-minor. Now $r(M \backslash \ell \backslash y)=r(M \backslash \ell)=r(M)=r(M \backslash y)$. Hence $\ell \in \mathrm{cl}_{M \backslash y}(X \cup(Y-y))$ and $M \backslash \ell \backslash y$ is 2-connected. Next we show the following.

91.8. Every non-trivial 2-separation of $M \backslash y$ has the form $\left(X \cup Y^{\prime} \cup \ell, Y^{\prime \prime}\right)$ where $Y^{\prime}$ and $Y^{\prime \prime}$ are disjoint and $Y^{\prime} \cup Y^{\prime \prime}=Y-y$.

Let $(A, B)$ be a non-trivial 2-separation of $M \backslash y$ that is not in the stated form. Without loss of generality, $\ell \in A$. Then $X \nsubseteq A$. Since $M \backslash \ell \backslash y$ is 2 -connected having the same rank as $M \backslash y$, it follows that $r(A-\ell)=r(A)$ and $(A-\ell, B)$ is a 2-separation of $M \backslash \ell \backslash y$. We also know that $(X, Y-y)$ is a 2-separation of $M \backslash \ell \backslash y$. Now $\ell \notin \operatorname{cl}(X)$ and $\ell \notin \operatorname{cl}(Y-y)$. But $\ell \in \operatorname{cl}(A-\ell)$, so $(A-\ell) \cap(Y-y) \neq \emptyset \neq(A-\ell) \cap X$. By uncrossing, $\lambda_{M \backslash \ell \backslash y}(B \cap X)=1$. As $\ell \in \operatorname{cl}(A-\ell)$ and $y \in \operatorname{cl}(Y-y)$, we deduce that $\lambda_{M}(B \cap X)=1$. Thus $B \cap X$ consists of a single point $x$ of $M$. Then $B \cap(Y-y) \neq \emptyset$. Therefore, by uncrossing again, $\lambda_{M \backslash \ell \backslash y}(X \cap(A-\ell))=1$, so $\lambda_{M \backslash \ell}(X \cap(A-\ell))=1$. Thus $(X-x, Y \cup x)$ is a 2-separation of $M \backslash \ell$. If $r(Y \cup x)=r(Y)$, then $x$ is parallel to $p$ in $M_{X}$. Hence, we see that $x$ lies on $y$. Then $M \backslash x$ is 3 -connected having a special $N$-minor, a contradiction. Thus we may assume that $r(Y \cup x)=r(Y)+1$. Then $r(X-x)=r(X)-1$. Hence, in $M_{X}$, the points $p$ and $x$ are a series pair. Thus $M_{X}$ is the 2-sum with basepoint $q$ of a 2-polymatroid $M_{X}^{\prime}$, say, and a copy of $U_{2,3}$ with ground set $\{q, p, x\}$. Moreover, every element of $Y$ is a 
line through $p$ in $M_{Y}$. Thus we see that both $M \backslash y$ and $M / y$ have special $N$-minors, a contradiction. We conclude that 91.8 holds, so (iii) of the lemma holds, and the proof of the lemma is complete.

Next we determine what happens when (ii) of Lemma 90 holds.

Lemma 92. Suppose $M \rrbracket \ell$ has $N$ as a c-minor. Let $(X, Y)$ be a 2-separation of $M \backslash \ell$ in which $X$ is the $N$-side and $|Y| \geqslant 3$. Let $M_{X} \oplus_{2} M_{Y}$ be the associated 2-sum decomposition of $M \backslash \ell$ with respect to the basepoint $p$. Then

(i) Y contains a doubly labelled element; or

(ii) $\sqcap(Y-y, X)>0$ for some $y$ in $Y$; or

(iii) $r\left(X \cup \ell \cup y_{0}\right)>r\left(X \cup y_{0}\right)$ for some $y_{0}$ in $Y$, and $M / y_{0}$ has a special $N$-minor. Moreover, either

(a) every non-trivial 2-separation of $M / y_{0}$ has the form $\left(Z_{1}, Z_{2}\right)$ where $Z_{1}$ is the $N$-side and $Z_{2} \subseteq Y-y_{0}$; or

(b) $M_{X}$ is the 2-sum with basepoint $q$ of two 2-polymatroids, one of which is a copy of $U_{2,3}$ with ground set $\{p, z, q\}$.

Proof. Assume that neither (i) nor (ii) holds. Suppose $y \in Y$. As $\sqcap(Y, X)=1$, it follows that $r(Y)>r(Y-y)$ so

92.1. $r(Y-y) \leqslant r(Y)-1$.

Next we show that

92.2. $\lambda_{M_{Y}}(\{y\})=\lambda_{M \backslash(X \cup \ell)}(\{y\})+1$.

We see that $\lambda_{M_{Y}}(\{y\})=r_{M}(\{y\})+r_{M_{Y}}((Y-y) \cup p)-r\left(M_{Y}\right)$. Since $\sqcap(Y-y, X)=0$, we deduce that $r_{M_{Y}}((Y-y) \cup p)=r_{M}(Y-y)+1$. As $M_{Y}$ is 2-connected, $r(Y)=r\left(M_{Y}\right)$ and 92.2 follows.

We now extend 92.1 as follows.

92.3. Let $\left\{y_{1}, y_{2}, \ldots, y_{k}\right\}$ be a subset of $Y$. Then

$$
r\left(Y-\left\{y_{1}, y_{2}, \ldots, y_{k}\right\}\right) \leqslant r(Y)-k .
$$

By 92.1, $r\left(Y-y_{1}\right) \leqslant r(Y)-1$ and $r\left(Y-y_{2}\right) \leqslant r(Y)-1$. Thus, by submodularity, $r\left(Y-\left\{y_{1}, y_{2}\right\}\right) \leqslant r(Y)-2$. Repeating this argument gives 92.3.

Next we show the following.

92.4. For all $y$ in $Y$, the 2-polymatroid $M \backslash \ell / y$ has a special $N$-minor and $\lambda_{M \backslash \ell / y}(X)=1$.

Let $M^{\prime}=M \backslash \ell$. By Corollary 33,

$$
\begin{aligned}
\lambda_{M^{\prime} / y}(X) & =\lambda_{M^{\prime} \backslash y}(X)-\sqcap_{M^{\prime}}(X, y)-\sqcap_{M^{\prime}}(Y-y, y)+r(\{y\}) \\
& =\lambda_{M^{\prime} \backslash y}(X)-r_{M^{\prime}}(Y-y)+r_{M^{\prime}}(Y) \text { as } \sqcap\left(X, Y-y^{\prime}\right)=0 \text { for all } y^{\prime} \text { in } Y ; \\
& =r_{M^{\prime}}(Y)-r_{M^{\prime}}(Y-y) .
\end{aligned}
$$


But

$$
\begin{aligned}
1 & =\lambda_{M^{\prime}}(X) \\
& =r(X)+r(Y)-r\left(M^{\prime}\right) \\
& \geqslant r(X \cup y)-r(\{y\})+r(Y)-r(\{y\})-r\left(M^{\prime}\right)+r(\{y\}) \\
& =\lambda_{M^{\prime} / y}(X) .
\end{aligned}
$$

We conclude, using (31) that, since $r(Y) \neq r(Y-y)$, we have $\lambda_{M^{\prime} / y}(X)=1$ for all $y$ in $Y$. Then, by Lemma 51(ii), $M^{\prime} / y$ has a special $N$-minor. Hence $M \backslash \ell / y$ has a special $N$-minor, that is, 92.4 holds.

92.5. If $y \in Y$ and $\ell$ is in a parallel pair of points in $M / y$, then $r(X \cup \ell \cup y)=r(X \cup y)$.

To see this, observe that, as $M$ is 3-connected, $\ell \notin \mathrm{cl}_{M}(Y)$. Thus $\ell$ is parallel to a point of $X$ in $M / y$, and 92.5 follows.

92.6. Let $Y=\left\{y_{1}, y_{2}, \ldots, y_{n}\right\}$. If $r\left(X \cup \ell \cup y_{i}\right)=r\left(X \cup y_{i}\right)$ for all $i$ in $\{1,2, \ldots, n\}$, then $\left\{y_{n-1}, y_{n}\right\}$ is a prickly 3-separator of $M$, and $M \downarrow y_{n}$ is 3-connected having a special $N$-minor.

First observe that each $y_{i}$ in $Y$ is a line for if $y_{i}$ is a point, then

$$
r\left(X \cup \ell \cup y_{i}\right)=r\left(X \cup y_{i}\right)=r(X)+r\left(\left\{y_{i}\right\}\right)=r(X)+1 .
$$

As $r\left(Y-y_{i}\right) \leqslant r(Y)-1$, we deduce that $\left(X \cup \ell \cup y_{i}, Y-y_{i}\right)$ is a 2-separation of $M$, a contradiction.

Continuing with the proof of 92.6 , next we show the following.

92.7. For $1 \leqslant k \leqslant n-1$,

$$
\begin{aligned}
r\left(X \cup \ell \cup\left\{y_{1}, y_{2}, \ldots, y_{k}\right\}\right) & =r(X)+1+k \text { and } \\
r\left(Y-\left\{y_{1}, y_{2}, \ldots, y_{k}\right\}\right) & =r(Y)-k .
\end{aligned}
$$

We argue by induction on $k$. By assumption, $r\left(X \cup \ell \cup y_{1}\right)=r(X)+r\left(\left\{y_{1}\right\}\right)=r(X)+2$. Moreover, $r\left(Y-y_{1}\right) \leqslant r(Y)-1$. Equality must hold otherwise we get the contradiction that $\left(X \cup \ell \cup y_{1}, Y-y_{1}\right)$ is a 2-separation of $M$. We deduce that the result holds for $k=1$. Assume it holds for $k<m$ and let $k=m \geqslant 2$. Then

$$
\begin{aligned}
r\left(X \cup\left\{y_{1}, y_{2}, \ldots, y_{m-1}\right\} \cup \ell\right)+r\left(X \cup\left\{y_{2}, y_{3}, \ldots, y_{m}\right\} \cup \ell\right) \\
\\
\geqslant r\left(X \cup\left\{y_{2}, y_{3}, \ldots, y_{m-1}\right\} \cup \ell\right)+r\left(X \cup\left\{y_{1}, y_{2}, \ldots, y_{m}\right\} \cup \ell\right) .
\end{aligned}
$$

If $m=2$, then $r\left(X \cup\left\{y_{2}, y_{3}, \ldots, y_{m-1}\right\} \cup \ell\right)=r(X \cup \ell) \geqslant r(X)+1$. If $m>2$, then $r\left(X \cup\left\{y_{2}, y_{3}, \ldots, y_{m-1}\right\} \cup \ell\right)=r(X)+m-1$ by the induction assumption. Thus

$$
\begin{aligned}
r\left(X \cup\left\{y_{1}, y_{2}, \ldots, y_{m}\right\} \cup \ell\right) & \leqslant r(X)+m+r(X)+m-(r(X)+m-1) \\
& =r(X)+m+1 .
\end{aligned}
$$


But

$$
r\left(Y-\left\{y_{1}, y_{2}, \ldots, y_{m}\right\}\right) \leqslant r(Y)-m .
$$

It follows that equality must hold in (32) and (33). Thus, by induction, 92.7 holds.

By 92.7, $r\left(Y-\left\{y_{1}, y_{2}, \ldots, y_{n-1}\right\}\right)=r(Y)-(n-1)$. But $r\left(Y-\left\{y_{1}, y_{2}, \ldots, y_{n-1}\right\}\right)=$ $r\left(\left\{y_{n}\right\}\right)=2$. Thus $r(Y)=n+1$, and it follows by 92.7 that $r\left(\left\{y_{n-1}, y_{n}\right\}\right)=3$ and $\left\{y_{n-1}, y_{n}\right\}$ is a prickly 3 -separating set in $M$. Hence, by Lemma $54, M \downarrow y_{n}$ is 3-connected. Recall that

$$
r_{M \downarrow y_{n}}(Z)= \begin{cases}r(Z), & \text { if } r\left(Z \cup y_{n}\right)>r(Z) ; \text { and } \\ r(Z)-1, & \text { otherwise. }\end{cases}
$$

Thus

$$
\begin{aligned}
\sqcap_{M \downarrow y_{n}}\left(X, Y-y_{n}\right) & =r_{M \downarrow y_{n}}(X)+r_{M \downarrow y_{n}}\left(Y-y_{n}\right)-r_{M \downarrow y_{n}}\left(X \cup\left(Y-y_{n}\right)\right) \\
& =r(X)+r\left(Y-y_{n}\right)-r(M)+1 \\
& =r(X)+r(Y)-r(M) \text { by } 92.7 ; \\
& =1 .
\end{aligned}
$$

It follows by Lemma 46 (vi) that $(M \backslash \ell) \downarrow y_{n}=M_{X} \oplus_{2} M_{Y} \downarrow y_{n}$. Then, by Lemma 51(iii), $(M \backslash \ell) \downarrow y_{n}$ has a special $N$-minor. We deduce that $M \downarrow y_{n}$ is 3 -connected having a special $N$-minor. Thus 92.6 holds.

Since we have assumed that the theorem fails, it follows, by 92.6, that, for some element $y_{0}$ of $Y$,

$$
r\left(X \cup \ell \cup y_{0}\right)>r\left(X \cup y_{0}\right) .
$$

By $92.4, M / y_{0}$ has a special $N$-minor. Thus $M / y_{0}$ is not 3 -connected. Moreover, by 92.5 , the element $\ell$ is not in a pair of parallel points of $M / y_{0}$.

Let $(A \cup \ell, B)$ be a 2 -separation of $M / y_{0}$ with $\ell \notin A$. Next we show that 92.8. $(A, B)$ is an exact 2-separation of $M / y_{0} \backslash \ell$, and $\ell \in \mathrm{cl}_{M / y_{0}}(A)$.

If $(A, B)$ is not exactly 2-separating in $M / y_{0} \backslash \ell$, then, by Proposition $47, M_{Y} / y_{0}$ is not 2-connected, so we obtain the contradiction that $Y$ contains a doubly labelled element. Thus $r_{M / y_{0}}(A \cup \ell)=r_{M / y_{0}}(A)$ and 92.8 holds.

We shall show that

92.9. either (iii)(b) holds, or $(A, B)$ does not cross $\left(X, Y-y_{0}\right)$.

Assume each of $A$ and $B$ meets each of $X$ and $Y-y_{0}$. Then, by uncrossing, $\lambda_{M \backslash \ell / y_{0}}(X \cap$ $B)=1$. But $\sqcap\left(X,\left\{y_{0}\right\}\right)=0$, so $r_{M}(X \cap B)=r_{M / y_{0}}(X \cap B)$. Also $r_{M}\left(\left(Y-y_{0}\right) \cup A \cup \ell \cup y_{0}\right)=$ $r_{M / y_{0}}\left(\left(Y-y_{0}\right) \cup A \cup \ell\right)+r\left(\left\{y_{0}\right\}\right)$. Then

$$
\begin{aligned}
& r(X \cap B))+r\left(\left(Y-y_{0}\right) \cup A \cup \ell \cup y_{0}\right)-r(M) \\
& =r_{M / y_{0}}(X \cap B)+r_{M / y_{0}}\left(\left(Y-y_{0}\right) \cup A \cup \ell\right)+r\left(\left\{y_{0}\right\}\right)-r\left(M / y_{0}\right)-r\left(\left\{y_{0}\right\}\right) \\
& =\lambda_{M / y_{0}}(X \cap B) \\
& =\lambda_{M / y_{0} \backslash \ell}(X \cap B) \quad \text { as } \ell \in \mathrm{cl}_{M / y_{0}}(A) ; \\
& =1 .
\end{aligned}
$$


Since $M$ is 3-connected, it follows that $X \cap B$ consists of a point $z$ of $M$.

Now $\lambda_{M \backslash \ell / y_{0}}\left(\left(Y-y_{0}\right) \cup z\right)=1$, so

$$
\begin{aligned}
1 & =r_{M / y_{0}}\left(\left(Y-y_{0}\right) \cup z\right)+r_{M / y_{0}}(A \cap X)-r\left(M / y_{0}\right) \\
& =r(Y \cup z)-r\left(\left\{y_{0}\right\}\right)+r\left((A \cap X) \cup y_{0}\right)-r\left(\left\{y_{0}\right\}\right)-r(M)+r\left(\left\{y_{0}\right\}\right) \\
& =r(Y \cup z))+r(A \cap X)-r(M \backslash \ell) \text { since } \sqcap\left(X,\left\{y_{0}\right\}\right)=0 .
\end{aligned}
$$

Thus $Y \cup z$ is 2-separating in $M \backslash \ell$. If $r(Y \cup z)=r(Y)$, then $z$ is parallel to the basepoint $p$ of the 2-sum. Hence each element of $Y$ is doubly labelled, a contradiction. Thus we may assume that $r(Y \cup z)=r(Y)+1$. Then $r(X-z)=r(X)-1$. Now $M_{X}$ is 2-connected, so $r\left(M_{X}\right)=r(X)$ and $M_{X}$ has $\{p, z\}$ as a series pair of points. It follows that $M_{X}$ is the 2-sum with basepoint $q$ of a 2-polymatroid $M_{X}^{\prime}$ and a copy of $U_{2,3}$ with ground set $\{q, z, p\}$. Thus (iii)(b) of the lemma holds. Hence so does 92.9.

We shall now assume that (iii)(b) does not hold.

92.10. $A \nsubseteq Y-y_{0}$ and $B \nsubseteq X$ and $A \nsubseteq X$.

To see this, first suppose that $A \subseteq Y-y_{0}$. Then, as $\ell \in \mathrm{cl}_{M / y_{0}}(A)$, we deduce that $\ell \in \operatorname{cl}_{M}(Y)$, a contradiction. Thus $A \nsubseteq Y-y_{0}$.

Now suppose that $B \subseteq X$. We have

$$
\begin{aligned}
1 & =\lambda_{M / y_{0}}(B) \\
& =r_{M / y_{0}}(B)+r_{M / y_{0}}(A \cup \ell)-r\left(M / y_{0}\right) \\
& =r\left(B \cup y_{0}\right)-r\left(\left\{y_{0}\right\}\right)+r\left(A \cup \ell \cup y_{0}\right)-r\left(\left\{y_{0}\right\}\right)-r(M)+r\left(\left\{y_{0}\right\}\right) \\
& =r(B)+r\left(A \cup \ell \cup y_{0}\right)-r(M) \text { as } B \subseteq X .
\end{aligned}
$$

Thus $\left(A \cup \ell \cup y_{0}, B\right)$ is a 2 -separation of $M$, a contradiction. Thus $B \nsubseteq X$.

Next suppose that $A \subseteq X$. As $(A \cup \ell, B)$ is a 2-separation of $M / y_{0}$, we have

$$
\begin{aligned}
1 & =r_{M / y_{0}}(A \cup \ell)+r_{M / y_{0}}(B)-r\left(M / y_{0}\right) \\
& =r\left(A \cup \ell \cup y_{0}\right)-r\left(\left\{y_{0}\right\}\right)+r\left(B \cup y_{0}\right)-r\left(\left\{y_{0}\right\}\right)-r(M)+r\left(\left\{y_{0}\right\}\right) \\
& \geqslant r\left(A \cup y_{0}\right)-r\left(\left\{y_{0}\right\}\right)+r\left(B \cup y_{0}\right)-r(M) \\
& \geqslant r(A)+r\left(B \cup y_{0}\right)-r(M \backslash \ell) \text { as } A \subseteq X ; \\
& \geqslant 1 \text { as } M \backslash \ell \text { is 2-connected. }
\end{aligned}
$$

We deduce that equality holds throughout, so $r\left(A \cup \ell \cup y_{0}\right)=r\left(A \cup y_{0}\right)$. But $A \subseteq X$, so $r\left(X \cup \ell \cup y_{0}\right)=r\left(X \cup y_{0}\right)$, contradicting the choice of $y_{0}$. Hence $A \nsubseteq X$, so 92.10 holds.

By 92.9 , we deduce that $B \subseteq Y-y_{0}$. Since, by $92.4, M / y_{0}$ has a special $N$-minor, we see that (iii)(a) of the lemma holds, so the lemma is proved.

We now combine the above lemmas to prove the following result. This lemma and its corollary are the main results of this section.

Lemma 93. Suppose $M \backslash \ell$ has $N$ as a c-minor. Let $(X, Y)$ be a 2-separation of $M \backslash \ell$ having $X$ as the $N$-side and $|Y|=\mu(\ell)$. Then $Y$ contains a doubly labelled element. 
Proof. By Lemma 87, $|Y| \geqslant 3$. Assume that $Y$ does not contain a doubly labelled element. Then, by Lemma 91,

(i)(a) $\sqcap(\{y\}, X) \neq 1$ for some $y$ in $Y$; or

(i)(b) $Y$ contains an element $y$ such that $M \backslash y$ has $N$ as a c-minor and every non-trivial 2-separation of $M \backslash y$ has the form $\left(Z_{1}, Z_{2}\right)$ where $Z_{1}$ is the $N$-side and $Z_{2} \subseteq Y-y$.

Now, since $|Y|=\mu(\ell)$, outcome (iii)(b) of Lemma 92 does not arise. Thus, by that lemma and Lemma 89,

(ii)(a) $\sqcap(Y-y, X)>0$ for some $y$ in $Y$; or

(ii)(b) $Y$ contains an element $y$ such that $M / y$ has $N$ as a c-minor and every non-trivial 2-separation of $M / y$ has the form $\left(Z_{1}, Z_{2}\right)$ where $Z_{1}$ is the $N$-side and $Z_{2} \subseteq Y-y$.

By Lemma 90, (i)(a) and (ii)(a) cannot both hold. Thus (i)(b) or (ii)(b) holds. Therefore, for some $y$ in $Y$, either $M \backslash y$ has $N$ as a c-minor and has a 2 -separation $\left(Z_{1}, Z_{2}\right)$ where $Z_{1}$ is the $N$-side, $Z_{2} \subseteq Y-y$, and $\left|Z_{2}\right|=\mu(y)<\mu(\ell)$, or $M / y$ has $N$ as a c-minor and has a 2-separation $\left(Z_{1}, Z_{2}\right)$ where $Z_{1}$ is the $N$-side, $Z_{2} \subseteq Y-y$, and $\left|Z_{2}\right|=\mu^{*}(y)<\mu(\ell)$. We can now repeat the argument above using $\left(y, Z_{2}\right)$ in place of $(\ell, Y)$ and, in the latter case, $M^{*}$ in place of $M$. Since we have eliminated the possibility that $\mu(\ell)=2$ or $\mu^{*}(\ell)=2$, after finitely many repetitions of this argument, we obtain a contradiction that completes the proof.

Corollary 94. The 2-polymatroid $M$ contains a doubly labelled line.

Proof. Take $\ell$ in $E(M)-E(N)$. Then $M \backslash \ell$ or $M / \ell$ has $N$ as a c-minor, so applying the last lemma to $M$ or its dual gives that $M$ has a doubly labelled element. By Lemma 58, this element is a line.

\section{Non- $N$-3-separators exist}

The purpose of this section is prove the existence of a non- $N$-3-separating set in $M$ where we recall that such a set $Y$ is exactly 3 -separating, meets $E(N)$ in at most one element, and, when it has exactly two elements, both of these elements are lines. The following lemma will be key in what follows.

Lemma 95. Let $(X, Y)$ be a 2-separation of $M \backslash \ell$ where $X$ is the $N$-side, $|Y| \geqslant 2$, and $Y$ is not a series pair of points in $M \backslash \ell$. Then $Y$ contains no points.

Proof. Assume that $Y$ contains a point $y$. Then, by Lemma 58, $y$ is not doubly labelled. 95.1. $M \backslash y$ or $M / y$ has a special $N$-minor.

To see this, consider the 2-connected 2-polymatroid $M_{Y}$. By Lemma 36, $M_{Y} \backslash y$ or $M_{Y} / y$ is 2-connected, so $\sqcap_{M \backslash y}(X, Y-y)=1$ or $\sqcap_{M / y}(X, Y-y)=1$. As $M_{X}$ has a special $N$-minor, so does $M \backslash y$ or $M / y$. 
95.2. $M \backslash y$ does not have a special $N$-minor.

Assume $M \backslash y$ does have a special $N$-minor. Then, as $y$ is not doubly labelled, $M / y$ does not have a special $N$-minor. Then, by Lemma 51(ii), $\Pi_{M / y}(X, Y-y)=0$, that is, $r_{M / y}(X)+r_{M / y}(Y-y)-r(M / y)=0$, so $r(X \cup y)+r(Y)=r(M)+r(\{y\})=r(M)+1$. But $r(X)+r(Y)=r(M)+1$, so $r(X \cup y)=r(X)$ and $r(Y-y)=r(Y)$ otherwise $(X \cup y, Y-y)$ is a 1-separation of $M \backslash \ell$, a contradiction to Lemma 59. Since $y \in Y$ and $r(X \cup y)=r(X)$, we see that $\sqcap(X,\{y\})=1$. But $\sqcap(X, Y)=1$. Thus, in $M_{Y}$, the point $y$ is parallel to the basepoint $p$ of the 2-sum. Hence $M \backslash \ell \backslash y$ is 2-connected and $r(M \backslash \ell \backslash y)=r(M)$.

Let $(A \cup \ell, B)$ be a non-trivial 2-separation of $M \backslash y$ where $\ell \notin A$. Now

$$
\begin{aligned}
1 & \leqslant r(A)+r(B)-r(M \backslash \ell, y) \\
& \leqslant r(A \cup \ell)+r(B)-r(M \backslash y) \\
& =1 .
\end{aligned}
$$

Thus $r(A)=r(A \cup \ell)$. Hence $\ell \in \operatorname{cl}(A)$ so $r(A) \geqslant 2$. Continuing with the proof of 95.2, we now show the following.

95.3. $(A, B)$ crosses $(X, Y-y)$.

Because $y \in \operatorname{cl}(X) \cap \operatorname{cl}(Y-y)$ but $y \notin \operatorname{cl}(A) \cup \operatorname{cl}(B)$, we deduce that neither $A$ nor $B$ contains $X$ or $Y-y$, so 95.3 holds.

By uncrossing, $\lambda_{M \backslash \ell, y}(B \cap(Y-y))=1$. But $\ell \in \operatorname{cl}(A)$ and $y \in \operatorname{cl}(X)$ so $\lambda_{M}(B \cap(Y-$ $y))=1$. Hence $B \cap(Y-y)$ consists of a single point, say $z$. As $z$ is not parallel to $y$, we deduce that $\sqcap(X,\{z\})=0$. Thus, by Lemma $29, \sqcap_{M / z}(X, Y-z)=\lambda_{M \backslash \ell / z}(X)=1$. Hence, by Lemma 51(ii), $M \backslash \ell / z$, and hence $M / z$, has a special $N$-minor. On the other hand,

$$
1=\sqcap(X,\{y\}) \leqslant \sqcap(X, Y-z) \leqslant \sqcap(X, Y)=1 .
$$

Thus $\sqcap_{M \backslash z}(X, Y-z)=1$ so $M \backslash z$ has a special $N$-minor. Since $z$ is a point, we have a contradiction to Lemma 58 that proves 95.2.

By combining 95.1 and 95.2 , we deduce that $M / y$ has a special $N$-minor but $M \backslash y$ does not. Since $(M, N)$ is a counterexample, $M / y$ is not 3-connected. By Lemma 59, $M / y$ is 2-connected.

As $M \backslash y$ does not have a special $N$-minor, by Lemma $51(\mathrm{i}), \sqcap(X, Y-y)=0$. But $\sqcap(X, Y)=1$. As $y$ is a point, it follows that

$$
r(Y-y)=r(Y)-1
$$

and $r(X \cup(Y-y))=r(X \cup Y)$. Moreover, as $(X \cup y, Y-y)$ is not a 1-separation of $M \backslash \ell$, we deduce that

95.4. $r(X \cup y)=r(X)+1$.

Now $r\left(M_{Y} \backslash p, y\right)=r(Y-y)=r(Y)-1$. But $r\left(M_{Y} \backslash p\right)=r(Y)$. If $r\left(M_{Y} \backslash y\right)=r(Y)-1$, then $\{y\}$ is a 1-separating set in $M_{Y}$. We deduce that $\{p, y\}$ is a series pair of points in $M_{Y}$. Thus $M_{Y} \backslash y$ is not 2-connected but $M_{Y}$ is, so, by Lemma 36, $M_{Y} / y$ is 2-connected. Hence, by Proposition 47, $M \backslash \ell / y$ is 2-connected. 
95.5. $(\{\ell\}, X \cup(Y-y))$ is not a 2-separation of $M / y$.

Assume the contrary. Then $r(\{\ell, y\})+r(X \cup Y)=r(M)+2$. But $r_{M / y}(\{\ell\})=2$ otherwise we do not have a 2-separation. Thus $r(\{\ell, y\})=3$, so $(\{\ell\}, X \cup Y)$ is a 2separation of $M$, a contradiction. Therefore 95.5 holds.

Let $(A \cup \ell, B)$ be a 2 -separation of $M / y$ with $\ell$ not in $A$. By 95.5, $A \neq \emptyset$. Since $M / y \backslash \ell$ is 2-connected, $\lambda_{M / y \backslash \ell}(A)>0$. Hence $\lambda_{M / y \backslash \ell}(A)=1$, so $\ell \in \mathrm{cl}_{M / y}(A)$. Hence one easily checks that

95.6. (i) $r(A \cup y \cup \ell)=r(A \cup y)$; and

(ii) $r(A \cup y)+r(B \cup y)=r(M \backslash \ell)+2$.

Next we show that

95.7. $(A, B)$ crosses $(X, Y-y)$.

Assume $B \cap(Y-y)=\emptyset$ or $B \cap X=\emptyset$. As $r(X \cup y)=r(X)+1$ and $r(Y)=r(Y-y)+1$, we have $r(B \cup y)=r(B)+1$. Then, as $r(A \cup y \cup \ell)=r(A \cup y)$, we have, by 95.6,

$$
r(A \cup y \cup \ell)+r(B)=r(M)+1,
$$

that is, $(A \cup y \cup \ell, B)$ is a 2-separation of $M$, a contradiction. We deduce that $B \cap(Y-y) \neq$ $\emptyset \neq B \cap X$.

Now assume that $A \cap(Y-y)=\emptyset$. Then $A \subseteq X$ and $Y-y \subseteq B$, so $r(X \cup y \cup \ell)=r(X \cup y)$. As $r(X \cup y)=r(X)+1$ and $r(Y-y)=r(Y)-1$, it follows that $(X \cup y \cup \ell, Y-y)$ is 2separating in $M$. Hence $Y-y$ consists of a single point $z$. Now $r(X)+r(Y)=r(M \backslash \ell)+1$, so $r(X)=r(M \backslash \ell)-1$. As $M \backslash \ell$ is connected, neither $y$ nor $z$ is $\operatorname{in} \operatorname{cl}(X)$ so $\{y, z\}$ is a series pair of points in $M \backslash \ell$, a contradiction. Hence $A \cap(Y-y) \neq \emptyset$.

Finally, assume that $X \cap A=\emptyset$. Then $A \subseteq Y-y$, so, as $r(A \cup y \cup \ell)=r(A \cup y)$, it follows that $r(Y \cup \ell)=r(Y)$, so $(X, Y \cup \ell)$ is a 2-separation of $M$, a contradiction. We conclude that 95.7 holds.

Next we determine the structure of the set $B$.

95.8. In $M$, the set $B$ consists of two points, $x^{\prime}$ and $y^{\prime}$, that lie in $B \cap X$ and $B \cap(Y-y)$, respectively.

By uncrossing, $\lambda_{M \backslash \ell / y}(X \cap B)=1$, so

$$
r((X \cap B) \cup y)+r(A \cup Y)-r(M \backslash \ell)=2 .
$$

As $X \cap B \subseteq X$, we deduce that $r((X \cap B) \cup y)=r(X \cap B)+1$. Also $y \in Y$, so $r(A \cup Y)=r(A \cup Y \cup \ell)$. Thus $(X \cap B, A \cup Y \cup \ell)$ is 2-separating in $M$. Hence $X \cap B$ consists of a point, say $x^{\prime}$.

By uncrossing again, we see that $\lambda_{M \backslash \ell / y}((Y-y) \cap B)=1$, so

$$
r(((Y-y) \cap B) \cup y)+r(A \cup X \cup y)-r(M \backslash \ell)=2 .
$$

Thus

$$
r((Y-y) \cap B)+r(A \cup X \cup y \cup \ell)=r(M)+1
$$


since $r(((Y-y) \cap B) \cup y)=r((Y-y) \cap B)+1$ and $r(A \cup X \cup y)=r(A \cup X \cup y \cup \ell)$. Hence $((Y-y) \cap B, A \cup X \cup y \cup \ell)$ is 2-separating in $M$, so $(Y-y) \cap B$ consists of a single matroid point, $y^{\prime}$. We deduce that 95.8 holds.

95.9. The element $y^{\prime}$ is doubly labelled.

To see this, first observe that, in $M / y$, the set $B$ is a 2 -separating set consisting of two matroid points, $x^{\prime}$ and $y^{\prime}$. Suppose $r_{M / y}(B)=2$. Then $r_{M / y}(A \cup \ell)=r(M / y)-1$, so $r(A \cup \ell \cup y)=r(M)-1$. Hence $\left(A \cup \ell \cup y,\left\{x^{\prime}, y^{\prime}\right\}\right)$ is a 2-separation of $M$, a contradiction. We deduce that $r_{M / y}(B)=1$ so $\left\{x^{\prime}, y^{\prime}\right\}$ is a pair of parallel points in $M / y$. Then $M / y \backslash y^{\prime}$, and so $M \backslash y^{\prime}$, has a special $N$-minor.

Now $r_{M / y}\left(\left\{x^{\prime}, y^{\prime}\right\}\right)=1$, so $r\left(\left\{x^{\prime}, y^{\prime}, y\right\}\right)=2$. Thus $y \in \mathrm{cl}_{M / y^{\prime}}(X)$, so $r\left(X \cup y^{\prime} \cup y\right)=$ $r\left(X \cup y^{\prime}\right)$. But, by 95.4, $r(X \cup y)>r(X)$, so $r\left(X \cup y^{\prime}\right)>r(X)$. Hence $\sqcap\left(X,\left\{y^{\prime}\right\}\right)=0$. Thus, by Lemma $29, \sqcap_{M / y^{\prime}}\left(X, Y-y^{\prime}\right)=\sqcap(X, Y)=1$. We conclude by Lemma 51 that $M / y^{\prime}$ has a special $N$-minor. Therefore 95.9 holds.

As 95.9 contradicts Lemma 58, we deduce that Lemma 95 holds.

Lemma 96. There is a c-minor $N_{0}$ of $M$ that is isomorphic to $N$ such that $M$ has a non- $N_{0}-3$-separating set.

Proof. By Corollary 94, $M$ has a doubly labelled line $\ell$. Moreover, by Lemma 59, each of $M \backslash \ell$ and $M / \ell$ is 2-connected.

Assume the lemma fails. Let $N_{D}$ and $N_{C}$ be special $N$-minors of $M \backslash \ell$ and $M / \ell$, respectively. We now apply what we have learned earlier using $N_{D}$ in place of $N$. Let $(X, Y)$ be a 2-separation of $M \backslash \ell$ in which $X$ is the $N_{D}$-side and $|Y|=\mu(\ell)$. Then $|Y| \geqslant 3$. Now $\sqcap(X,\{\ell\}) \in\{0,1\}$.

We show next that

96.1. $\sqcap(X,\{\ell\})=0$ and $\sqcap(Y,\{\ell\})=0$.

Assume that $\sqcap(X,\{\ell\})=1$. Then $r(X \cup \ell)=r(X)+1$, so $\lambda_{M}(Y)=2$. Thus $Y$ is a non- $N_{D}$-3-separating set, a contradiction. Thus $\sqcap(X,\{\ell\})=0$. Similarly, if $\sqcap(Y,\{\ell\})=1$, then $\lambda_{M}(X)=2$, so $Y \cup \ell$ is a non- $N_{D^{-}}$-3-separating set. This contradiction completes the proof of 96.1 .

We deduce that $M \backslash \ell$ has a 2 -separation $\left(D_{1}, D_{2}\right)$ where $D_{1}$ is the $N_{D}$-side, $\left|D_{2}\right|=$ $\mu(\ell) \geqslant 3$, and $\sqcap\left(D_{1}, \ell\right)=0=\sqcap\left(D_{2}, \ell\right)$. A similar argument to that used to show 96.1 shows that $M / \ell$ has a 2 -separation $\left(C_{1}, C_{2}\right)$ where $C_{1}$ is the $N_{C^{-}}$side, $\left|C_{2}\right|=\mu^{*}(\ell) \geqslant 3$, and $\sqcap\left(C_{1}, \ell\right)=2=\sqcap\left(C_{2}, \ell\right)$. We observe here that the definition of $\mu^{*}(\ell)$ depends on $N_{C}$ here rather than on $N_{D}$.

By the local connectivity conditions between $\ell$ and each of $D_{1}, D_{2}, C_{1}$, and $C_{2}$, 96.2. $\left(C_{1}, C_{2}\right)$ and $\left(D_{1}, D_{2}\right)$ cross.

We have $r\left(D_{1}\right)+r\left(D_{2}\right)=r(E-\ell)+1$ and $r\left(C_{1}\right)+r\left(C_{2}\right)=r(E-\ell)+3$. By uncrossing,

$$
\lambda_{M \backslash \ell}\left(D_{2} \cap C_{2}\right)+\lambda_{M \backslash \ell}\left(D_{1} \cap C_{1}\right) \leqslant 4 .
$$

Suppose $\lambda_{M \backslash \ell}\left(D_{2} \cap C_{2}\right) \leqslant 1$. Since $\ell \in \operatorname{cl}\left(D_{1} \cup C_{1}\right)$, it follows that $\lambda_{M}\left(D_{2} \cap C_{2}\right) \leqslant 1$. Thus $D_{2} \cap C_{2}$ consists of a single point, $z$. Then

$$
2=\sqcap\left(C_{2},\{\ell\}\right) \leqslant \sqcap\left(D_{1} \cup z,\{\ell\}\right) \leqslant \sqcap\left(D_{1},\{\ell\}\right)+1=1,
$$


a contradiction. We deduce that $\lambda_{M \backslash \ell}\left(D_{2} \cap C_{2}\right)=2=\lambda_{M \backslash \ell}\left(D_{1} \cap C_{1}\right)$, so $\lambda_{M}\left(D_{2} \cap C_{2}\right)=$ $2=\lambda_{M}\left(D_{1} \cap C_{1}\right)$. By symmetry, $\lambda_{M}\left(D_{1} \cap C_{2}\right)=2=\lambda_{M}\left(D_{2} \cap C_{1}\right)$.

Clearly each of $D_{2} \cap C_{1}$ and $D_{2} \cap C_{2}$ contains at most one element of $N_{D}$. As $\left|D_{2}\right| \geqslant 3$, we deduce from Lemma 95 that $D_{2}$ contains no points. Hence, some $Z$ in $\left\{D_{2} \cap C_{1}, D_{2} \cap C_{2}\right\}$ contains at least two elements. Then $Z$ is a non- $N_{D^{-}} 3$-separator of $M$.

For the rest of the proof of Theorem 57, we will use the c-minor $N_{0}$ of $M$ found in the last lemma. To avoid cluttering the notation, we will relabel $N_{0}$ as $N$.

Lemma 97. Let $Y_{1}$ be a minimal non- $N$-3-separating set in $M$ with $\left|Y_{1}\right| \geqslant 3$, and let $X_{1}=E(M)-Y_{1}$. Suppose $\ell$ is an element of $Y_{1}$ such that $M \backslash \ell$ has $N$ as a c-minor. Let $(A, B)$ be a 2-separation of $M \backslash \ell$ where $A$ is the $N$-side and $|B|=\mu(\ell)$. Then one of the following holds.

(i) $\lambda_{M \backslash \ell}\left(Y_{1}-\ell\right)=1$; or

(ii) $B \subseteq Y_{1}-\ell$; or

(iii) $(A, B)$ crosses $\left(X_{1}, Y_{1}-\ell\right)$ and $\lambda_{M \backslash \ell}\left(A \cap\left(Y_{1}-\ell\right)\right)=1=\lambda_{M \backslash \ell}\left(B \cap\left(Y_{1}-\ell\right)\right)$, while $\lambda_{M \backslash \ell}\left(A \cap X_{1}\right)=2=\lambda_{M \backslash \ell}\left(B \cap X_{1}\right)=\lambda_{M \backslash \ell}\left(Y_{1}-\ell\right)$.

Proof. Assume neither (i) nor (ii) holds. Then $\ell \in \operatorname{cl}\left(Y_{1}-\ell\right)$ and $B \nsubseteq Y_{1}-\ell$. If $B \subseteq X_{1}$, then $\lambda_{M}(B)=1$, a contradiction. If $B \supseteq Y_{1}-\ell$, then $\lambda_{M}(A)=1$, a contradiction. Finally, observe that $\left|X_{1} \cap A\right| \geqslant 2$ since $|E(N)| \geqslant 4$ and $X_{1}$ and $A$ are the $N$-sides of their separations. We conclude that $(A, B)$ crosses $\left(X_{1}, Y_{1}-\ell\right)$.

By Lemma 87, $|B| \geqslant 3$. By Lemma $95, B$ contains no points. Now $\lambda_{M \backslash \ell}\left(B \cap X_{1}\right) \geqslant 2$ otherwise, as $\ell \in \mathrm{cl}\left(Y_{1}-\ell\right)$, we get the contradiction that $\lambda_{M}\left(B \cap X_{1}\right)=1$. By uncrossing, we deduce that $\lambda_{M \backslash \ell}\left(A \cap\left(Y_{1}-\ell\right)\right) \leqslant 1$. Since $\left|X_{1} \cap A\right| \geqslant 2$, we get, similarly, that $\lambda_{M \backslash \ell}\left(A \cap X_{1}\right) \geqslant 2$, so $\lambda_{M \backslash \ell}\left(B \cap\left(Y_{1}-\ell\right)\right) \leqslant 1$. As $M \backslash \ell$ is 2 -connected, we deduce that $\lambda_{M \backslash \ell}\left(A \cap\left(Y_{1}-\ell\right)\right)=1=\lambda_{M \backslash \ell}\left(B \cap\left(Y_{1}-\ell\right)\right)$. Hence $\lambda_{M \backslash \ell}\left(A \cap X_{1}\right)=2=\lambda_{M \backslash \ell}\left(B \cap X_{1}\right)$. We conclude that (iii) holds. Hence so does the lemma.

\section{Finding big enough 3-separators}

In this section, we first establish 63, which is restated in the next lemma. We also start the proof of 65. That proof will be completed in Section 12 .

Lemma 98. $M$ has a minimal non-N-3-separator with at least three elements.

Proof. Assume every minimal non- $N$-3-separating set has exactly two elements. Let $\{a, b\}$ be such a set, $Z$. Then both of its members are lines. We may assume that $b \notin E(N)$. Suppose first that $r(Z)=2$. Then $a$ and $b$ are parallel lines. Suppose that $N$ is a c-minor of $M / b$. Since $a$ is a loop of $M / b$, we deduce that $a \notin E(N)$ so $M \backslash a$ has $N$ as a c-minor. Since $M \backslash a$ is 3 -connected, this is a contradiction. We may now assume that $M \backslash b$ has $N$ as a c-minor. Since it is 3 -connected, we have a contradiction that implies that $r(Z)>2$. 
Suppose next that $r(Z)=4$. Then $r^{*}(Z)=\|Z\|+r(E-Z)-r(M)=4-2=2$. Hence $Z$ consists of a pair of parallel lines in $M^{*}$, so we obtain a contradiction as above. We may now assume that $r(Z)=3$. Then $Z$ is a prickly 3 -separating set and, by Lemma 54 , $M \downarrow b$ is 3-connected. Hence $M \downarrow b$ has no c-minor isomorphic to $N$.

Now $M \backslash b$ or $M / b$ has $N$ as a c-minor. We begin by assuming the former. Let $(S \cup a, T)$ be a non-trivial 2-separation of $M \backslash b$ with $a \notin S$. Suppose the non- $N$-side of $(S \cup a, T)$ has $\mu(b)$ elements. By Lemma 87, $\mu(b) \geqslant 3$. We have $r(S \cup a)+r(T)-r(M)=1$. As $\sqcap(\{a\},\{b\})=1$ and $M$ is 3-connected, $r(S \cup a \cup b)=r(S \cup a)+1$, so

$$
\lambda_{M}(T)=2 .
$$

Moreover,

$$
r(S \cup a) \geqslant r(S)+1
$$

otherwise $r(S \cup a)=r(S)$ so $r(E-b)=r(E-\{a, b\})$, a contradiction.

Next we show the following.

98.1. Suppose $M \backslash b$ has a 2-separation $\left(S_{1}, S_{2}\right)$ where $S_{1}$ is the $N$-side and $S_{2}$ contains a prickly 3-separator $\{u, v\}$ where $u \notin E(N)$. Then $M \backslash b \downarrow u$ is not 2-connected.

Suppose $M \backslash b \downarrow u$ is 2-connected. Now $M \backslash b=M_{1} \oplus_{2} M_{2}$ where $M_{i}$ has ground set $S_{i} \cup p$. Since $M \backslash b \downarrow u$ is 2-connected, $\sqcap_{M \backslash b \downarrow u}\left(S_{1}, S_{2}-u\right)=1$. Then, by Lemma 51 (iii), $M \backslash b \downarrow u$ has a special $N$-minor. By Lemma 54, $M \downarrow u$ is 3 -connected. Since it has a c-minor isomorphic to $N$, we have a contradiction. Thus 98.1 holds.

Now suppose that $T$ is the $N$-side of $(S \cup a, T)$. Then, by Lemma 95, $S \cup a$ contains no points. Assume that $r(S \cup a)=r(S)+1$. As $r(S \cup a)+r(T)-r(M \backslash b)=1$, we see that

$$
[r(S)+1]+r(T)-[r(M \backslash b, a)+1]=1 .
$$

Hence $\sqcap(S, T)=1$, so, by Lemma $51(\mathrm{i}), M \backslash b \backslash a$ has a special $N$-minor. As $\{a, b\}$ is a prickly 3-separating set, we see that $M \backslash b \backslash a=M \downarrow b \backslash a$ so $M \downarrow b$ has a c-minor isomorphic to $N$, a contradiction.

Next we consider the case when $T$ is the $N$-side of $(S \cup a, T)$, and $r(S \cup a)=r(S)+2$. Then $r(S)+r(T \cup a \cup b)=r(M)+2$. Thus $S$ is a non- $N$-3-separator and so contains a minimal such set, $\{u, v\}$ where $u \notin E(N)$. From above, we know that $\{u, v\}$ is a prickly 3-separator of $M$. By 98.1, $M \backslash b \downarrow u$ is not 2-connected. Now $M \backslash b \downarrow u=M \downarrow u \backslash b$. Let $(J, K)$ be a 1 -separation of $M \downarrow u \backslash b$ with $a \in J$. Then $r_{M \downarrow u}(J \cup b) \leqslant r_{M \downarrow u}(J)+1$. Thus

$$
\begin{gathered}
r_{M \downarrow u}(J \cup b)+r_{M \downarrow u}(K)-r(M \downarrow u) \leqslant\left[r_{M \downarrow u}(J)+r_{M \downarrow u}(K)-r(M \downarrow u \backslash b)\right] \\
+[1+r(M \downarrow u \backslash b)-r(M \downarrow u)] \\
=1+r(M \downarrow u \backslash b)-r(M \downarrow u) .
\end{gathered}
$$

By Lemma 54, $M \downarrow u$ is 3-connected, so $r(M \downarrow u \backslash b)=r(M \downarrow u)$, and $K$ consists of a 
single point, $k$, of $M \downarrow u$. Then

$$
\begin{aligned}
1 & =r_{M \downarrow u}(J)+r_{M \downarrow u}(\{k\})-r(M \downarrow u \backslash b) \\
& =r_{M \downarrow u}(E-\{b, u, k\})+r_{M \downarrow u}(\{k\})-r(M \downarrow u) \\
& =r(E-\{b, k\})-1+r(\{k\})-r(M)+1 \\
& =r(E-\{b, k\})+r(\{k\})-r(M \backslash b) .
\end{aligned}
$$

Hence $\{k\}$ is 1 -separating in $M \backslash b$. Thus $k$ contradicts Lemma 58 .

When $M \backslash b$ has $N$ as a c-minor, it remains to consider the case when $S \cup a$ is the $N$-side of $(S \cup a, T)$. As $\mu(b) \geqslant 3$, it follows that $|T| \geqslant 3$. By $(34), \lambda_{M}(T)=2$. By assumption, $T$ contains a minimal non- $N$-3-separating set $T^{\prime}$. The latter consists of a pair, $\{u, v\}$, of lines that form a prickly 3 -separating set. We may assume that $u \notin E(N)$. Now $M \backslash b$ is certainly 2-connected. By Lemma 54, $M \downarrow u$ is 3-connected. Since $\sqcap(\{a\},\{b\})=1$, it follows that $M \downarrow u \backslash b$ is 2 -connected, a contradiction. We conclude that $M \backslash b$ does not have a c-minor isomorphic to $N$.

We now know that $M / b$ has $N$ as a c-minor. Moreover, $M^{*}$ has $N^{*}$ as a c-minor and has $\{a, b\}$ as a prickly 3-separating set; and $(M / b)^{*}=\left(M^{*} \backslash b\right)^{b}$. This means we can use $\left(M^{*}, N^{*}, M^{*} \backslash b\right)$ in place of $(M, N, M \backslash b)$ in the argument above to complete the proof of the lemma.

The argument to establish that $M$ has a minimal non- $N$-3-separator with at least four elements is much longer than that just given since it involves analyzing a number of cases. We shall use three preliminary results. In each, we denote $E(M)-Y_{1}$ by $X_{1}$.

Lemma 99. Let $Y_{1}$ be a minimal non- $N$-3-separator with exactly three elements. Suppose $\ell \in Y_{1}$ and $M \backslash \ell$ has $N$ as a c-minor. Let $(A, B)$ be a 2-separation of $M \backslash \ell$ where $A$ is the $N$-side and $|B| \geqslant 3$. Suppose $\ell \in \operatorname{cl}\left(Y_{1}-\ell\right)$. Then $(A, B)$ crosses $\left(X_{1}, Y_{1}-\ell\right)$ and $\lambda_{M \backslash \ell}\left(X_{1} \cap A\right) \geqslant 2$. Moreover, $Y_{1} \cap B$ consists of a single line.

Proof. As $\ell \in \operatorname{cl}\left(Y_{1}-\ell\right)$, we see that $\lambda_{M_{1} \backslash \ell}\left(Y_{1}-\ell\right)=2$. To see that $(A, B)$ crosses $\left(X_{1}, Y_{1}-\ell\right)$, note first that, as $\left|Y_{1}-\ell\right|=2$ and $|A|,|B| \geqslant 3$, neither $A$ nor $B$ is contained in $Y_{1}-\ell$. Moreover, $Y_{1}-\ell$ is not contained in $A$ or $B$ otherwise $(A \cup \ell, B)$ or $(A, B \cup \ell)$ is a 2-separation of $M$, a contradiction. Hence $(A, B)$ crosses $\left(X_{1}, Y_{1}-\ell\right)$.

As $|E(N)| \geqslant 4$, we see that $\left|X_{1} \cap A\right| \geqslant 2$. Then

$$
\lambda_{M \backslash \ell}\left(X_{1} \cap A\right) \geqslant 2
$$

otherwise, as $\ell \in \operatorname{cl}\left(Y_{1}-\ell\right)$, we get the contradiction that $\lambda_{M}\left(X_{1} \cap A\right) \leqslant 1$. By uncrossing, $\lambda_{M \backslash \ell}\left(Y_{1} \cap B\right) \leqslant 1$. By Lemma $95, B$ contains no points, so $Y_{1} \cap B$ contains no points. As $\left|Y_{1}-\ell\right|=2$, we see that $Y_{1} \cap B$ consists of a single line.

Lemma 100. Let $Y_{1}$ be a minimal non- $N$-3-separator with exactly three elements. If $Y_{1}$ contains a line $\ell$ such that $M \backslash \ell$ has $N$ as a c-minor, then $Y_{1}$ consists of three lines.

Proof. Assume that the lemma fails. Let $(A, B)$ be a 2 -separation of $M \backslash \ell$ where $A$ is the $N$-side and $|B| \geqslant 3$. First we show that 
100.1. $\ell \in \operatorname{cl}\left(Y_{1}-\ell\right)$.

Assume that $\ell \notin \operatorname{cl}\left(Y_{1}-\ell\right)$. Then $\left(X_{1}, Y_{1}-\ell\right)$ is a 2-separation of $M \backslash \ell$ with $\left|Y_{1}-\ell\right|=2$. By Lemma 95, we may assume that $Y_{1}-\ell$ consists of a series pair $\left\{y_{1}, y_{2}\right\}$ of points. Now $r(M \backslash \ell)=r(M)=r\left(X_{1}\right)+1$, so $r\left(\left\{\ell, y_{1}, y_{2}\right\}\right)=3$. Moreover, for each $i$ in $\{1,2\}$, we see that $M \backslash \ell / y_{i}$, and hence $M / y_{i}$, has a special $N$-minor.

As the theorem fails for $M$, we know that $M / y_{i}$ is not 3 -connected. Now $M / y_{i}$ is certainly 2-connected. Let $(J, K)$ be a 2-separation of it where we may assume that $\ell \in J$. Now $r_{M / y_{i}}\left(\left\{\ell, y_{j}\right\}\right)=2$ where $\{i, j\}=\{1,2\}$.

Suppose $r_{M / y_{i}}(\{\ell\})=2$. Assume $y_{j} \in K$. Then $\left(J \cup y_{j}, K-y_{j}\right)$ is a 2-separation of $M / y_{i}$ unless $K-y_{j}$ consists of a single point. In the exceptional case, $y_{j}$ is in a parallel pair of points in $M / y_{i}$. Hence $M \backslash y_{j}$ has a special $N$-minor. As $M / y_{j}$ also has such a minor, we contradict Lemma 58. We deduce that we may assume that $J$ contains $\left\{\ell, y_{j}\right\}$. Then $r\left(J \cup y_{i}\right)+r\left(K \cup y_{i}\right)=r(M)+2$, so $r\left(J \cup y_{i}, K\right)$ is a 2-separation of $M$, a contradiction.

We may now assume that $r_{M / y_{i}}(\{\ell\})=1$. Then $y_{i}$ lies on the line $\ell$. Since this must be true for each $i$ in $\{1,2\}$, we see that $r\left(\left\{\ell, y_{1}, y_{2}\right\}\right)=2$, a contradiction. We deduce that 100.1 holds.

By Lemma 99, we know that $(A, B)$ crosses $\left(X_{1}, Y_{1}\right)$, that $\lambda_{M \backslash \ell}\left(X_{1} \cap A\right) \geqslant 2$, and that $Y_{1} \cap B$ consists of a single line. As the lemma fails, $A \cap\left(Y_{1}-\ell\right)$ consists of a single point, $a$. As $\lambda_{M \backslash \ell}\left(X_{1} \cap A\right) \geqslant 2$ and $\lambda_{M \backslash \ell}(A)=1$, we deduce that $r(A-a)=r(A)$ and $r(B \cup a)=r(B)+1$. Hence $a \in \operatorname{cl}\left(X_{1}\right)$. Thus $Y_{1}-a$ is a minimal non- $N$-3-separator, a contradiction.

The next lemma verifies 64 .

Lemma 101. Let $Y_{1}$ be a minimal non-N-3-separator having exactly three elements. Then $Y_{1}$ consists of three lines.

Proof. As $\left|Y_{1} \cap E(N)\right| \leqslant 1$, at least two of the elements of $Y_{1}$ are not in $E(N)$. Let $\ell$ be one of these elements. Suppose $\ell$ is a line. If $M \backslash \ell$ has $N$ as a c-minor, then the result follows by Lemma 100. If $M / \ell$ has $N$ as a c-minor, then $\left(M^{*} \backslash \ell\right)^{b}$, and hence $M^{*} \backslash \ell$ has $N^{*}$ as a c-minor and again the result follows by Lemma 100 .

We may now assume that $\ell$ is a point. By switching to the dual if necessary, we may assume that $M \backslash \ell$ has $N$ as a c-minor. Let $(A, B)$ be a 2 -separation of $M \backslash \ell$ where $A$ is the $N$-side and $|B| \geqslant 3$. Next we show that

101.1. $\ell \notin \operatorname{cl}\left(Y_{1}-\ell\right)$.

Assume $\ell \in \operatorname{cl}\left(Y_{1}-\ell\right)$. Then, by Lemma 99, we know that $(A, B)$ crosses $\left(X_{1}, Y_{1}-\ell\right)$, that $\lambda_{M \backslash \ell}\left(X_{1} \cap A\right) \geqslant 2$, and that $Y_{1} \cap B$ consists of a single line, say $m$. Now $\left|B \cap X_{1}\right| \geqslant 2$ since $|B| \geqslant 3$. Then

$$
\lambda_{M \backslash \ell}\left(B \cap X_{1}\right) \geqslant 2
$$

otherwise, since $\ell \in \operatorname{cl}\left(Y_{1}-\ell\right)$, we deduce that $\lambda_{M}\left(B \cap X_{1}\right) \geqslant 1$, a contradiction. By uncrossing, $\lambda_{M \backslash \ell}\left(Y_{1} \cap A\right) \leqslant 1$.

Since $\left|Y_{1}\right|=3$ and $Y_{1} \cap B$ consists of the line $m$, we deduce that $A \cap\left(Y_{1}-\ell\right)$ consists of a single point, say $a$, otherwise one of the elements of $Y_{1}-\ell$ is a line that is not in 
$E(N)$ and we have already dealt with that case. As $\lambda_{M \backslash \ell}\left(X_{1} \cap A\right) \geqslant 2$ and $\lambda_{M \backslash \ell}(A)=1$, we deduce that

$$
r(A-a)=r(A) \text { and } r(B \cup a)=r(B)+1 .
$$

Hence

$$
a \in \operatorname{cl}\left(X_{1}\right) .
$$

We may assume that $m \in E(N)$ otherwise $m$ is removed in forming $N$ and that case was dealt with in the first paragraph.

Now $Y_{1}=\{a, \ell, m\}$. As $m \in B$, it follows by (35) that $r(\{m, a\})=3$. Moreover, as $\{m, a\}=Y_{1}-\ell$ and $\ell \in \operatorname{cl}\left(Y_{1}-\ell\right)$, we deduce that $r\left(Y_{1}\right)=3$. By $(36), r\left(X_{1} \cup a\right)=r\left(X_{1}\right)$. We deduce that

$$
r(\{a, \ell, m\})=r(\{\ell, m\})=3 \text { and } r\left(X_{1} \cup a\right)=r(M)-1 .
$$

Since $m \in E(N)$, it follows that $a \notin E(N)$. Suppose that $M \backslash \ell / a$ has $N$ as a c-minor. Still as part of the proof of 101.1, we show next that

101.2. $M / a$ is the 2-sum with basepoint $q$ of two 2-polymatroids, one of which consists of the line $m$ having non-parallel points $q$ and $\ell$ on it.

By $(37),\left(\{\ell, m\}, X_{1}\right)$ is a 2 -separation of $M / a$. Thus $M / a$ is the 2 -sum with basepoint $q$ of two 2-polymatroids, one of which, $Q$ say, consists of the line $m$ having points $q$ and $\ell$ on it. Suppose $q$ and $\ell$ are parallel points in $Q$. Then $\left(\{m\}, X_{1} \cup \ell\right)$ is a 2-separation of $M / a$. It follows that $\left(\{m\}, X_{1} \cup \ell \cup a\right)$ is a 2-separation of $M$, a contradiction. Thus 101.2 holds.

By 101.2, both $M / \ell$ and $M \backslash \ell$ have $N$ as a c-minor, a contradiction to Lemma 58 .

We now know that $N$ is a c-minor of $M \backslash \ell \backslash a$. In that 2-polymatroid, $\{m\}$ is 2separating so, in the formation of $N$, the element $m$ is compactified. As the next step towards showing 101.1, we now show that

101.3. $M \downarrow \underline{ } m$ is 3 -connected.

To see this, it will be helpful to consider the 2-polymatroid $M_{1}$ that is obtained from $M$ by freely adding the point $m^{\prime}$ on $m$. By definition, $M \downarrow m=M_{1} / m_{1}$. Certainly $M_{1}$ is 3 -connected, so $M_{1} / m^{\prime}$ is 2-connected. Assume it has a 2-separation $(U, V)$ where $m \in U$. Then

$$
r\left(U \cup m^{\prime}\right)+r\left(V \cup m^{\prime}\right)-r\left(M_{1}\right)=2 .
$$

But $r\left(U \cup m^{\prime}\right)=r(U)$. Hence $r\left(V \cup m^{\prime}\right)=r(V)$ otherwise $M_{1}$ has a 2-separation, a contradiction. But, as $m^{\prime}$ was freely placed on $m$, we deduce that $r\left(V \cup m^{\prime} \cup m\right)=$ $r\left(V \cup m^{\prime}\right)=r(V)$. Now, in $M_{1} \backslash m$, we see that $\left\{\ell, m^{\prime}\right\}$ is a series pair of points. As $m^{\prime} \in \mathrm{cl}(V)$, it follows that $\ell \in V$. Then $r(U-m)<r(U)$ since $\{m\}$ is 2-separating in $M \backslash \ell$. Now $r(U-m)=r(U)-1$ otherwise $r(U-m)=r(U)-2$ and $\left(U-m, V \cup\left\{m^{\prime}, m\right\}\right)$ is a 1-separation of $M_{1}$. As $\left(U-m, V \cup\left\{m^{\prime}, m\right\}\right)$ is not a 2-separation of $M_{1}$, it follows that $U-m$ consists of a single point $u$ and $r(\{u, m\})=2$. Thus, in $M \backslash \ell$, when we compactify $m$, we find that $u$ and $m$ are parallel. Since $m \in E(N)$, we see that $u \notin E(N)$. Moreover, $M \backslash u$ has $N$ as a c-minor. Since $u$ lies on $m$ in $M$, we deduce that $M \backslash u$ is 3-connected having $N$ as a c-minor. This contradiction completes the proof of 101.3. 
Now, in $M_{1} / m^{\prime}$, the elements $a, \ell$, and $m$ form a triangle of points. We know that $M_{1} / m^{\prime} \backslash \ell$ is not 3 -connected otherwise $(M \backslash \ell)^{b}$ is 3 -connected having $N$ as a c-minor. Because $M \backslash a$ has $N$ as a c-minor, $M \backslash a$ is not 3-connected, so $M_{1} \backslash a$ is not 3-connected. Still continuing with the proof of 101.1, we show next that

101.4. $M_{1} \backslash a / m^{\prime}$ is not 3 -connected.

Let $(G, H)$ be a 2-separation of $M_{1} \backslash a$ with $m$ in $G$. Then $\left(G \cup m^{\prime}, H-m^{\prime}\right)$ is a 2-separation of $M_{1} \backslash a$ unless $H$ consists of two points. In the exceptional case, $r(H)=2$ so $r(G)=r(M)-1$. But then $a \cup\left(H-m^{\prime}\right)$ is a series pair in $M$, a contradiction. We conclude that we may assume that $m^{\prime} \in G$. Then $\ell \in H$, otherwise, by (37), $(G \cup a, H)$ is a 2-separation of $M_{1}$, a contradiction.

Observe that $G \neq\left\{m, m^{\prime}\right\}$ otherwise $\{m\}$ is 2-separating in $M \backslash a$ and so, as $a \in \operatorname{cl}\left(X_{1}\right)$, we obtain the contradiction that $\{m\}$ is 2-separating in $M$.

Now

$$
r_{M_{1} \backslash a / m^{\prime}}\left(G-m^{\prime}\right)+r_{M_{1} \backslash a / m^{\prime}}(H)-r\left(M_{1} \backslash a / m^{\prime}\right)=r(G)+r\left(H \cup m^{\prime}\right)-1-r\left(M_{1} \backslash a\right) .
$$

Suppose that $r\left(H \cup m^{\prime}\right)=r(H)$. Then $r\left(H \cup m^{\prime} \cup m\right)=r(H)$ as $m^{\prime}$ is freely placed on $m$. Thus, as $G \supsetneqq\left\{m, m^{\prime}\right\}$ and $\{m\}$ is 2-separating in $M \backslash \ell \backslash a$, we see that $(G-m-$ $\left.m^{\prime}, H \cup\left\{m, m^{\prime}\right\}\right)$ is a 1 -separation of $M_{1} \backslash a$. Therefore $\left(G-m-m^{\prime}, H \cup\{m, a\}\right)$ is a 1-separation of $M$, a contradiction.

We now know that $r\left(H \cup m^{\prime}\right)=r(H)+1$. Then, as $(G, H)$ is a 2-separation of $M_{1} \backslash a$, it follows by (38) that $\left(G-m^{\prime}, H\right)$ is a 2-separation of $M_{1} \backslash a / m^{\prime}$ unless either $|H|=1$ and $r_{M_{1} / m^{\prime}}(H)=1$, or $\left|G-m^{\prime}\right|=1$ and $r_{M_{1} / m^{\prime}}\left(G-m^{\prime}\right)=1$. Consider the exceptional cases. The first of these cannot occur since $m^{\prime}$ is freely placed on $m$; the second cannot occur since it implies that $G=\left\{m, m^{\prime}\right\}$, which we eliminated above. As neither of the exceptional cases occurs, $M_{1} \backslash a / m^{\prime}$ has a 2-separation and so 101.4 holds.

Recall that $M_{1} / m^{\prime}=M \downarrow m$. In this 2-polymatroid, we have $\{a, \ell, m\}$ as a triangle such that the deletion of either $a$ or $\ell$ destroys 3 -connectedness. Hence, by Lemma 42 , there is a triad of $M_{1} / m^{\prime}$ that contains $a$ and exactly one of $\ell$ and $m$. Assume this triad contains $\ell$. Thus, in $M \backslash \ell \downarrow m$, we have that $a$ is in a series pair with some element $b$. Then $M \backslash \ell / a$ has $N$ as a c-minor, so $a$ is a doubly labelled point of $M$, a contradiction to Lemma 58. We deduce that $M_{1} / m^{\prime}$ has a triad containing $\{a, m\}$ but not $\ell$. Then $M_{1} / m^{\prime} \backslash \ell$, which equals $M \backslash \ell \downarrow m$, either has a triad containing $\{a, m\}$ or has $a$ in a series pair. This is straightforward to see by considering the matroid that is naturally derived from $M \backslash \ell \downarrow m$ and using properties of the cocircuits in this matroid. Now $a$ is not in a series pair in $M \backslash \ell \downarrow m$ otherwise we again obtain the contradiction that $a$ is a doubly labelled point. We deduce that $M \backslash \ell \downarrow m$ has a triad containing $\{a, m\}$. Since $m \in B$ and, by (35), $r(A-a)=r(A)$, we must have that the third point, $b$, of this triad is in $A-a$.

Now $M \backslash \ell \underline{\downarrow} m$ has $(A, B)$ as a 2-separation and has $\{a, b, m\}$ as a triad with $\{a, b\} \subseteq A$. Thus $(A \cup m, B-m)$ is a 2-separation of $M \backslash \ell \downarrow m$. Since $\ell$ is in the triangle $\{a, m, \ell\}$ in $M \downarrow m$, it follows that $(A \cup m \cup \ell, B-m)$ is a 2 -separation of $M \downarrow m$. This contradiction to 101.3 completes the proof of 101.1 .

Since $\ell \notin \operatorname{cl}\left(Y_{1}-\ell\right)$, we deduce that $\left(X_{1} \cup \ell, Y_{1}-\ell\right)$ is 3 -separating in $M$. By the argument in the first paragraph of the proof of the lemma, $Y_{1}-\ell$ does not consist of 
two lines; and, if it contains one line, that line is in $E(N)$. Moreover, $\left(X_{1}, Y_{1}-\ell\right)$ is a 2-separation in $M \backslash \ell$.

101.5. $Y_{1}-\ell$ does not consist of a point and a line.

Assume that $Y_{1}-\ell$ consists of a line $k$ and a point $y$. Then $k \in E(N)$, so $y \notin E(N)$. If $r\left(Y_{1}-\ell\right)=2$, then $M \backslash \ell \backslash y$, and hence $M \backslash y$, has $N$ as a c-minor. Since $y$ is on the line $k$, we see that $M \backslash y$ is 3 -connected, a contradiction. We deduce that $r\left(Y_{1}-\ell\right)=3$. Hence

$$
r\left(X_{1}\right)=r(M)-2 \text { and } r\left(X_{1} \cup \ell\right)=r(M)-1 .
$$

Now $M \backslash \ell$ is the 2-sum with basepoint $p$, say, of two 2-polymatroids, $M_{X}$ and $M_{Y}$, with ground sets $X_{1} \cup p$ and $\left(Y_{1}-\ell\right) \cup p$, respectively. Then $r\left(M_{Y}\right)=3$. Moreover, $y$ does not lie on $k$ in $M_{Y}$, otherwise $M_{Y}$ is not 2-connected, a contradiction to Proposition 47. Thus $M^{*} \backslash y$ has $N^{*}$ as a c-minor. Then, by applying 101.1 to $M^{*} \backslash y$, we deduce that $y \notin \mathrm{cl}_{M^{*}}\left(Y_{1}-y\right)$. Thus $r^{*}\left(Y_{1}-y\right)=r^{*}\left(Y_{1}\right)-1$. It follows that $r\left(X_{1} \cup \ell \cup y\right)=r\left(X_{1} \cup \ell\right)$. But $r\left(X_{1} \cup \ell \cup y\right)=r(M \backslash k)=r(M)$ yet $r\left(X_{1} \cup \ell\right)=r(M)-1$. This contradiction completes the proof of 101.5

We now know that $Y_{1}-\ell$ consists of a series pair of points, say $y_{1}$ and $y_{2}$. Now $r(M \backslash \ell)=r(M)=r\left(X_{1}\right)+1$. Also $r\left(\left\{\ell, y_{1}, y_{2}\right\}\right)=3$. Thus $\left\{\ell, y_{1}, y_{2}\right\}$ is a triad of $M$. Moreover, both $M / y_{1}$ and $M / y_{2}$ have special $N$-minors. Thus neither is 3 -connected. By Lemma $42, M$ has a triangle that contains $y_{1}$ and exactly one of $y_{2}$ and $\ell$. Likewise, $M$ has a triangle that contains $y_{2}$ and exactly one of $y_{1}$ and $\ell$. Thus either

(i) $M$ has a triangle $\left\{y_{1}, y_{2}, z\right\}$; or

(ii) $M$ has triangles $\left\{y_{1}, \ell, z_{1}\right\}$ and $\left\{y_{2}, \ell, z_{2}\right\}$ but no triangle containing $\left\{y_{1}, y_{2}\right\}$.

In the first case, $M / y_{1}$ has $\left\{y_{2}, z\right\}$ as a pair of parallel points. Hence $M \backslash y_{2}$ has a special $N$-minor. Thus $y_{2}$ is doubly labelled, a contradiction. We deduce that (ii) holds. Thus $M$ contains a fan $x_{1}, x_{2}, \ldots, x_{n}$ where $\left(x_{1}, x_{2}, x_{3}, x_{4}, x_{5}\right)=\left(z_{2}, y_{2}, \ell, y_{1}, z_{1}\right)$. Hence $M / x_{2}$ has a c-minor isomorphic to $N$. Then, by Lemmas 43 and 58, we obtain a contradiction.

We complete the proof of 65 by analyzing the various possibilities for a minimal non$N$-3-separator consisting of exactly three lines.

\section{Finding a minimal non- $N$-3-separator with at least four ele- ments}

In this section, we finish the proof of 65 . We begin by restating that assertion.

Lemma 102. $M$ has a minimal non-N-3-separator with at least four elements.

By Lemma 101, a minimal non- $N$-3-separator of $M$ with exactly three elements consists of three lines. To prove Lemma 102, we look at the number of these lines that have local connectivity one with the other side of the 3 -separator and then systematically eliminate all possibilities. 
We have $\left(X_{1}, Y_{1}\right)$ as a 3 -separation of the 3-connected 2-polymatroid $M$. We shall consider the extension $M+z$ of $M$ that is obtained by adjoining the line $z$ to $M$ so that $z$ is in the closure of each of $X_{1}$ and $Y_{1}$ in $M+z$. To see that this extension exists, we note that, by building on a result of Geelen, Gerards, and Whittle [3], Beavers [1, Proposition 2.2.2] showed that, when $(A, B)$ is a 3 -separation in a 3 -connected matroid $Q$, we can extend $Q$ by an independent set $\left\{z_{1}, z_{2}\right\}$ of size two so that these two points are clones, and each lies in the closure of both $A$ and $B$ in the extension $Q^{\prime}$. By working in the matroid naturally derived from $M$, we can add $z_{1}$ and $z_{2}$. This corresponds to adding the line $z$ to $M$ to form $M+z$ where $z=\left\{z_{1}, z_{2}\right\}$.

More formally, recall that the natural matroid $M^{\prime}$ derived from $M$ is obtained from $M$ by freely adding two points, $s_{\ell}$ and $t_{\ell}$, on each line $\ell$ of $M$ and then deleting all such lines $\ell$. After we have extended $M^{\prime}$ by $z_{1}$ and $z_{2}$, we have a matroid with points $\left\{z_{1}, z_{2}\right\} \cup\{p: p$ is a point of $M\} \cup\left\{s_{\ell}, t_{\ell}: \ell\right.$ is a line of $\left.M\right\}$. Taking $z=\left\{z_{1}, z_{2}\right\}$, we see that $M+z$ is the 2-polymatroid with elements $\{z\} \cup\{p: p$ is a point of $M\} \cup\{\ell$ : $\ell$ is a line of $M\}=\{z\} \cup E(M)$. We call $M+z$ the 2-polymatroid that is obtained from $M$ by adding the guts line $z$ of $\left(X_{1}, Y_{1}\right)$.

When we have $Y_{1}$ as a minimal non- $N$-3-separator of $M$ consisting of three lines, we look at $(M+z) \mid\left(Y_{1} \cup z\right)$. This 2-polymatroid consists of exactly four lines.

Lemma 103. $(M+z) \mid\left(Y_{1} \cup z\right)$ has no parallel lines, so $r_{M+z}\left(Y_{1} \cup z\right) \geqslant 3$.

Proof. Suppose $a$ and $b$ are parallel lines in $Y_{1}$. Then we may assume that $b \notin E(N)$. Now $M \backslash b$ or $M / b$ has $N$ as a c-minor. In the latter case, as $a$ is a loop of $M / b$, it follows that $a \notin E(N)$ and $M \backslash a$ has $N$ as a c-minor. We conclude that $M \backslash b$ or $M \backslash a$ has $N$ as a c-minor. Since each of $M \backslash b$ and $M \backslash a$ is 3-connected, we obtain the contradiction that the theorem holds. Thus $Y_{1}$ contains no pair of parallel lines.

Suppose $z$ is parallel to some element $y$ of $Y_{1}$. Then $\left(X_{1} \cup y, Y_{1}-y\right)$ is a non- $N-3$ separator of $M$ contradicting the minimality of $Y_{1}$. Thus $(M+z) \mid\left(Y_{1} \cup z\right)$ has no parallel lines and the lemma holds.

Lemma 104. $r\left(Y_{1}\right)>3$.

Proof. Assume that $r\left(Y_{1}\right)=3$. Then $r_{M+z}\left(Y_{1} \cup z\right)=3$, so $\sqcap(\{z\},\{y\})=1$ for all $y$ in $Y_{1}$. Moreover, $r\left(Y_{1}-y\right)=3=r\left(Y_{1}\right)$ for all $y$ in $Y_{1}$.

Suppose that $y \in Y_{1}-E(N)$ and $N$ is a c-minor of $M / y$. Then the remaining two elements, $y_{1}$ and $y_{2}$, of $Y$ are parallel points in $M / y$. We may assume that $y_{1} \notin E(N)$. Thus $M \backslash y_{1}$ has $N$ as a c-minor. We conclude that $N$ is a c-minor of $M \backslash y$ for some element $y$ of $Y_{1}$. We now focus on this element $y$.

Let $(R, G)$ be a non-trivial 2-separation of $M \backslash y$, that is, $\lambda_{M \backslash y}(R)=1$ and both $R$ and $G$ have at least two elements. We show next that

104.1. $(R, G)$ crosses $\left(X_{1}, Y_{1}-y\right)$.

If $R \subseteq X_{1}$, then $G \supseteq Y_{1}-y$ so $y \in \operatorname{cl}_{M}(G)$ and $(R, G \cup y)$ is a 2-separation of $M$. This contradiction implies, using symmetry, that both $R$ and $G$ meet $Y_{1}-y$.

Suppose $R \cap X_{1}=\emptyset$. Then $R$ consists of single line, so $(R, G)$ is a trivial 2-separation. This contradiction, combined with symmetry, completes the proof of 104.1 . 
Let $Y_{1}-y=\{a, b\}$. We may assume that $a \in R$ and $b \in G$. Now, as $y \in \operatorname{cl}\left(Y_{1}-y\right)$, we see that $\lambda_{M \backslash y}\left(Y_{1}-y\right)=2$. Thus

$$
\begin{aligned}
1+2 & =\lambda_{M \backslash y}(R)+\lambda_{M \backslash y}\left(Y_{1}-y\right) \\
& \geqslant \lambda_{M \backslash y}(\{a\})+\lambda_{M \backslash y}\left(R \cup\left(Y_{1}-y\right)\right) \\
& =\lambda_{M \backslash y}(\{a\})+\lambda_{M \backslash y}\left(G \cap X_{1}\right) .
\end{aligned}
$$

We know $r\left(E-Y_{1}\right)=r\left(X_{1}\right)=r(M)-1$ since $r\left(Y_{1}\right)=3$. Thus $r(E-\{y, a\})=$ $r\left(X_{1} \cup b\right)=r(M)$. Hence

$$
\lambda_{M \backslash y}(\{a\})=r(\{a\})+r(E-\{y, a\})-r(E-y)=r(\{a\})=2,
$$

so $\lambda_{M \backslash y}\left(G \cap X_{1}\right) \leqslant 1$. But $y \in \operatorname{cl}(\{a, b\})$ so $\lambda_{M}\left(G \cap X_{1}\right) \leqslant 1$. By symmetry, $\lambda_{M}\left(R \cap X_{1}\right) \leqslant$ 1. We conclude that $\left|G \cap X_{1}\right| \leqslant 1$ and $\left|R \cap X_{1}\right| \leqslant 1$, so $\left|X_{1}\right| \leqslant 2$. This is a contradiction since $|E(N)| \geqslant 4$. We conclude that the lemma holds.

Lemma 105. $r_{M+z}\left(Y_{1} \cup z\right)=r_{M}\left(Y_{1}\right)=4$.

Proof. We know that $r_{M+z}\left(Y_{1} \cup z\right)=r_{M}\left(Y_{1}\right) \geqslant 4$. Suppose $r_{M}\left(Y_{1}\right) \geqslant 5$. Then $r_{M}\left(X_{1}\right) \leqslant$ $r(M)-3$, so

$$
r_{M^{*}}\left(Y_{1}\right)=\sum_{y \in Y_{1}} r_{M}(\{y\})+r_{M}\left(X_{1}\right)-r(M) \leqslant 6+r(M)-3-r(M)=3 .
$$

By using $M^{*}$ in place of $M$, we get a contradiction to Lemma 104. We conclude that the lemma holds.

We will now work with the 2-polymatroid $(M+z) \mid\left(Y_{1} \cup z\right)$, which we rename $P$. This has rank 4 and consists of four lines.

Lemma 106. If $B \subseteq Y_{1}$ and $A=Y_{1}-B$, then

$$
\sqcap_{P}(A \cup z, B)=\sqcap_{M+z}\left(A \cup X_{1} \cup z, B\right) .
$$

Proof. Since $P=(M+z) \mid\left(Y_{1} \cup z\right)$, we can do all of these local connectivity calculations in $M+z$. Now $\sqcap\left(A \cup z, X_{1}\right)=\sqcap\left(A \cup z, X_{1} \cup z\right)$, so

$$
2=\sqcap\left(Y_{1} \cup z, X_{1}\right) \geqslant \sqcap\left(A \cup z, X_{1}\right)=\sqcap\left(A \cup z, X_{1} \cup z\right) \geqslant 2 .
$$

Thus

$$
r(A \cup z)-2=r\left(A \cup z \cup X_{1}\right)-r\left(X_{1}\right) .
$$

Hence

$$
\begin{aligned}
\sqcap(A \cup z, B) & =r(A \cup z)+r(B)-r(A \cup z \cup B) \\
& =r\left(A \cup z \cup X_{1}\right)-r\left(X_{1}\right)+2+r(B)-r\left(Y_{1}\right) \\
& =r\left(A \cup z \cup X_{1}\right)+r(B)-\left[r\left(X_{1}\right)+r\left(Y_{1}\right)-2\right] \\
& =r\left(A \cup z \cup X_{1}\right)+r(B)-r(M) \\
& =\sqcap\left(A \cup X_{1} \cup z, B\right) .
\end{aligned}
$$


Lemma 107. $P$ is 3-connected.

Proof. From the last lemma, if $(A, B)$ is a $k$-separation of $P$ for some $k$ in $\{1,2\}$ and $z \in A$, then $\left(A \cup X_{1} \cup z, B\right)$ is a $k$-separation of $M+z$, a contradiction.

Lemma 108. If $y \in Y_{1}$ and $\sqcap\left(X_{1},\{y\}\right)=1$, then $r\left(Y_{1}-y\right)=4$.

Proof. By Lemma 103, $r\left(Y_{1}-y\right)>2$. If $r\left(Y_{1}-y\right)=3$, then $\left(X_{1} \cup y, Y_{1}-y\right)$ is a 3 -separation violating the choice of $\left(X_{1}, Y_{1}\right)$.

Lemma 109. Suppose $y \in Y_{1}$ and $r\left(Y_{1}-y\right)=4$. If $m$ is a line such that $\{m\}$ is 2-separating in $M \backslash y$, then $m \in Y_{1}-y$.

Proof. We have $1=r(\{m\})+r(E-\{y, m\})-r(M \backslash y)$. Thus $r(E-\{y, m\})=r(M)-1$. Suppose $m \notin Y_{1}-y$. Then $E-\{y, m\}$ contains $Y_{1}-y$ and so spans $y$. Thus $r(E-\{y, m\})=$ $r(M \backslash m)=r(M)$, a contradiction.

The next four lemmas will help eliminate many of the possibilities for $P$. Let $a, b$, and $c$ be the lines in $P$ other than $z$.

Lemma 110. If $c$ is skew to $X_{1}$ in $M$, and $M / c$ has a and $b$ as parallel lines, then $M / c$ is 3-connected.

Proof. Assume $(A, B)$ is a $k$-separation of $M / c$ for some $k$ in $\{1,2\}$ where $|A| \leqslant|B|$. If $\{a, b\} \subseteq Z$ for some $Z$ in $\{A, B\}$, and $\{Z, W\}=\{A, B\}$, then $r(Z \cup c)+r(W \cup c)-r(M)=$ $k+1$. But $c$ is skew to $W$ since $W \subseteq X_{1}$, so $(Z \cup c, W)$ is a $k$-separation of $M$, a contradiction. We may now assume that $a \in A$ and $b \in B$. Then $(A \cup b, B-b)$ is a $k$ separation of $M / c$ with $\{a, b\} \subseteq A \cup b$ and this possibility has already been eliminated.

Lemma 111. If $c$ is skew to each of $a, b$, and $X_{1}$ in $M$, then $M / c$ has no $c$-minor isomorphic to $N$.

Proof. We see that $M / c$ has $a$ and $b$ as parallel lines. Since $(M, N)$ is a counterexample to the theorem, we obtain this lemma as a direct consequence of the last one.

Lemma 112. Assume that $M \backslash b$ has a c-minor isomorphic to $N$ and that $P \backslash b$ has rank 4, has c skew to each of $a$ and $z$, and has $\sqcap(\{a\},\{z\})=1$. Then $M / c$ has a c-minor isomorphic to $N$.

Proof. Let $(A, C)$ be a non-trivial 2-separation of $M \backslash b$. If $\{a, c\}$ is contained in $A$ or $C$, then $M$ has a 2-separation, a contradiction. Thus we may assume that $a \in A$ and $c \in C$. Because $c$ is skew to $z$ in $P$, and $C-c \subseteq X_{1}$ in $M \backslash b$, we see that $c$ is skew to $C-c$ in $M \backslash b$. Thus $(A \cup c, C-c)$ is 2-separating in $M \backslash b$. Hence $(A \cup c \cup b, C-c)$ is 2-separating in $M$. Thus $C-c$ consists of a point $d$ of $M$. Now, by Lemma 109, the only 2 -separating lines in $M \backslash b$ can be $a$ and $c$. But $a$ is not 2-separating. Thus $(M \backslash b)^{b}=M \backslash b \downarrow c$, so $c$ is a point of $M \backslash b \downarrow c$. The rank of this 2-polymatroid is $r(M)-1$, and it has $\{c, d\}$ as a series pair since $A$ has rank $r(M)-2$ in it. Thus $M \backslash b \downarrow c / c$, and hence $M / c$, has a c-minor isomorphic to $N$. 
Lemma 113. If $\sqcap(\{a\},\{z\})=1$ and both $b$ and $c$ are skew to each other and to $z$, then $M \backslash a$ has no c-minor isomorphic to $N$.

Proof. Assume that $M \backslash a$ has a c-minor isomorphic to $N$. Let $(B, C)$ be a $k$-separation of $M \backslash a$ for some $k$ in $\{1,2\}$. If $B$ or $C$ contains $\{b, c\}$, then $M$ has a $k$-separation. Thus we may assume that $b \in B$, that $c \in C$, and that $|B| \geqslant|C|$. As $b$ is skew to $z$ in $P$, we deduce that $b$ is skew to $B-b$ in $M \backslash a$. The partition $(B-b, C \cup b \cup a)$ of $E(M)$ now shows that $M$ is not 3 -connected, a contradiction.

By Lemma 103, for all $y$ in $Y_{1}$, we have $\sqcap(\{y\},\{z\}) \in\{0,1\}$. We shall treat the possibilities for $P$ based on the number $\theta$ of members $y$ of $Y_{1}$ for which $\sqcap(\{y\},\{z\})=1$. The most difficult case is when $\theta=3$ and we will treat that after we deal with the cases when $\theta=2$ and when $\theta=1$.

Lemma 114. $\theta \neq 2$.

Proof. Suppose that $\sqcap(\{a\},\{z\})=1=\sqcap(\{b\},\{z\})$ and $\sqcap(\{c\},\{z\})=0$. Then, by Lemma 108, $r(\{b, c\})=4=r(\{a, c\})$. Thus, by Lemma 111, $M / c$ has no c-minor isomorphic to $N$. By Lemma 112, neither $M \backslash a$ nor $M \backslash b$ has a c-minor isomorphic to $N$. Thus, without loss of generality, we may assume that $M / a$ has a c-minor isomorphic to $N$. Now, in $M / a$, we have $\{b, c\}$ as a 2-separating set where $c$ is a line and $b$ is either a point on that line or is a parallel line. Thus, by Lemma 51, $M / a \backslash b$, and hence $M \backslash b$, has a c-minor isomorphic to $N$, a contradiction.

We can exploit duality to eliminate the case when $\theta=1$.

Lemma 115. $\theta \neq 1$.

Proof. Suppose that $\sqcap(\{a\},\{z\})=1$ and $\sqcap(\{b\},\{z\})=0=\sqcap(\{c\},\{z\})$. Then, by Lemma 108, $r(\{b, c\})=4$. By Lemma 35, $\sqcap^{*}\left(\{y\}, X_{1}\right)=\lambda_{M /\left(Y_{1}-y\right)}(\{y\})$ for $y$ in $Y_{1}$. Since $\{b, c\}$ spans $a$ in $M$, we deduce that $\Pi^{*}\left(\{a\}, X_{1}\right)=0$. If $\Pi^{*}\left(\{b\}, X_{1}\right)=1=\Pi^{*}\left(\{c\}, X_{1}\right)$, then $\theta=2$ in $M^{*}$ so the result follows by Lemma 114 . Thus, we may assume, by symmetry, that $\Pi^{*}\left(\{b\}, X_{1}\right)=0$. Hence $\{a, c\}$ spans $b$ in $M$, so $r(\{a, c\})=4$. Thus, by Lemma 111, $M / c$ does not have a c-minor isomorphic to $N$. By Lemma 112, $M \backslash b$ has no c-minor isomorphic to $N$. If $\sqcap(\{a\},\{b\})=0$, then, by symmetry, the argument of the last two sentences shows that neither $M / b$ nor $M \backslash c$ has a c-minor isomorphic to $N$. Thus both $b$ and $c$ must be in every c-minor of $M$ isomorphic to $N$, a contradiction. We deduce that $\sqcap(\{a\},\{b\})=1$.

By Lemma 113, $M \backslash a$ has no c-minor isomorphic to $N$. Suppose $M / a$ has a c-minor isomorphic to $N$. In $M / a$, we see that $\{c, b\}$ is a 2-separating set with $c$ as a line and $b$ as a point on it. Hence, by Lemma $51, M / a \backslash b$, and so $M \backslash b$, has a c-minor isomorphic to $N$, a contradiction. We conclude that $M / a$ has no c-minor isomorphic to $N$. It follows that $a$ is in every c-minor of $M$ isomorphic to $N$. Thus $M / b$ has $N$ as a c-minor. In $M / b$, we see that $a$ is a point on the line $c$. Suppose that $a$ is parallel to some point $e$, say. 
Then $e \in X_{1}$. Moreover, $M / b \backslash e$, and hence $M \backslash e$, has a c-minor isomorphic to $N$. Now $r\left(X_{1} \cup\{a, b\}\right)=r\left(X_{1}\right)+2$. Thus

$$
\begin{aligned}
r\left(X_{1}\right)+1+3 & =r\left(X_{1} \cup a\right)+r(\{a, b, e\}) \\
& \geqslant r(\{a, e\})+r\left(X_{1} \cup\{a, b\}\right) \\
& =r(\{a, e\})+r\left(X_{1}\right)+2 .
\end{aligned}
$$

Hence $r(\{a, e\})=2$, so $e$ lies on $a$ in $M$. Thus $M \backslash e$ is 3 -connected having a c-minor isomorphic to $N$, a contradiction. We deduce that, in $M / b$, the point $a$ is not parallel to another point, so $M / b$ is simple.

We complete the proof by showing that $M / b$ is 3 -connected. Suppose it has $(A, C)$ as a 2-separation. If $A$ or $C$, say $A$, contains $\{a, c\}$, then $b$ is skew to $C$, so $(A \cup b, C)$ is a 2-separation of $M$, a contradiction. Thus, we may assume that $a \in A$ and $c \in C$. Then, as $a$ is a point on the line $c$ in $M / b$, we see that $(A-a, C \cup a)$ is 2 -separating in $M / b$. It is not a 2-separation otherwise we obtain a contradiction as before. It follows that $A$ is a parallel pair of points in $M / b$, contradicting the fact that $M / b$ is simple.

Next we eliminate the case when $\theta=3$. The core of the argument in this case mimics the argument used to prove Tutte's Triangle Lemma for matroids (see, for example, [14, Lemma 8.7.7]).

Lemma 116. $\theta \neq 3$.

Proof. Assume that $\Pi(\{a\},\{z\})=\sqcap(\{b\},\{z\})=\sqcap(\{c\},\{z\})=1$. Then, by Lemma 108, $r(\{a, b\})=r(\{b, c\})=r(\{a, c\})=4$. First we show the following.

116.1. $Y_{1}$ has at least two members $y$ such that $M \backslash y$ has a c-minor isomorphic to $N$.

Assume that this fails. Since $\left|Y_{1}-E(N)\right| \geqslant 2$, there is an element, say $a$, of $Y_{1}-E(N)$ such that $M / a$ has $N$ as a c-minor. In $M / a$, we see that $b$ and $c$ are parallel lines and $\{b, c\}$ is 2-separating. Thus, by Lemma 51 , each of $M / a \backslash b$ and $M / a \backslash c$ have special $N$-minors. This contradiction implies that 116.1 holds.

We now assume that both $M \backslash a$ and $M \backslash b$ have special $N$-minors. Clearly, $M \backslash a$ has $b$ and $c$ as 2-separating lines, and, by Lemma 109, these are the only 2-separating lines in $M \backslash a$. Thus $(M \backslash a)^{b}=M \backslash a \downarrow b \downarrow c$. Symmetrically, $(M \backslash b)^{b}=M \backslash b \downarrow a \downarrow c$. As the theorem fails, neither $(M \backslash a)^{b}$ nor $(M \backslash b)^{b}$ is 3-connected. Thus each of $M \downarrow c \backslash \bar{a}$ and $M \downarrow c \backslash b$ have non-trivial 2-separations. It will be convenient to work in the 2-polymatroid $M \bar{\downarrow} c$, which we shall rename $M_{c}$. Let $\left(X_{a}, Y_{a}\right)$ and $\left(X_{b}, Y_{b}\right)$ be non-trivial 2-separations of $\bar{M}_{c} \backslash a$ and $M_{c} \backslash b$, respectively, with $b$ in $Y_{a}$ and $a$ in $Y_{b}$.

Now it is straightforward to check the following.

116.2. If $Z \subseteq X_{1}$ and $e \in\{a, b\}$, then $\sqcap_{M}(Z,\{e\})=\sqcap_{M_{c}}(Z,\{e\})$.

We deduce that

116.3. $\sqcap_{M_{c}}\left(X_{1},\{a\}\right)=1=\sqcap_{M_{c}}\left(X_{1},\{b\}\right)$.

Next we show that

116.4. $c \in X_{a} \cap X_{b}$. 
Suppose $c$ in $Y_{a}$. Since $\{c, b\}$ spans $a$ in $M_{c}$, it follows that $\left(X_{a}, Y_{a} \cup a\right)$ is a 2-separation of $M_{c}$ and hence of $M$, a contradiction. Thus $c \in X_{a}$ and, by symmetry, 116.4 holds.

116.5. For $Z \subseteq X_{1}$, if $\sqcap_{M}(Z,\{a\})=1=\sqcap_{M}(Z,\{b\})$, then $\sqcap_{M}(Z,\{a, b\})=2$.

Assume $\sqcap_{M}(Z,\{a, b\})<2$. Then $\Pi_{M}(Z,\{a, b\})=\sqcap_{M}(Z,\{a\})=1$. Thus

$$
r(Z)+r(\{a, b\})-r(Z \cup\{a, b\})=r(Z)+r(\{a\})-r(Z \cup a),
$$

so $r(\{a, b\})-r(\{a\})=r(Z \cup\{a, b\})-r(Z \cup a)$. Hence $b$ is skew to $Z \cup a$, so $b$ is skew to $Z$, a contradiction. We deduce that 116.5 holds.

116.6. For $Z \subseteq X_{1}$, if $\sqcap_{M_{c}}(Z,\{a\})=1=\sqcap_{M_{c}}(Z,\{b\})$, then $\sqcap_{M_{c}}(Z,\{a, b\})=2$.

By $116.2, \sqcap_{M_{c}}(Z,\{a\})=\sqcap_{M}(Z,\{a\})$. Moreover,

$$
\begin{aligned}
\sqcap_{M_{c}}(Z,\{a, b\}) & =r_{M_{c}}(Z)+r_{M_{c}}(\{a, b\})-r_{M_{c}}(Z \cup\{a, b\}) \\
& =r_{M}(Z)+\left[r_{M}(\{a, b\})-1\right]-\left[r_{M}(Z \cup\{a, b\})-1\right] \\
& =\sqcap_{M}(Z,\{a, b\}) .
\end{aligned}
$$

Thus 116.6 follows immediately from 116.5.

116.7. Assume $Z \subseteq X_{1}$ and $\sqcap_{M_{c}}(Z,\{a, b\})=2$. Then $c \in \operatorname{cl}_{M_{c}}(Z)$.

To see this, note that

$$
r_{M_{c}}(Z \cup\{a, b, c\})=r_{M_{c}}(Z \cup\{a, b\})=r_{M_{c}}(Z)+r_{M_{c}}(\{a, b\})-2=r_{M_{c}}(Z)+1 .
$$

By submodularity,

$$
r_{M_{c}}(E-\{a, b\})+r_{M_{c}}(Z \cup\{a, b, c\}) \geqslant r\left(M_{c}\right)+r_{M_{c}}(Z \cup c) .
$$

Thus

$$
r\left(M_{c}\right)-1+r_{M_{c}}(Z)+1 \geqslant r\left(M_{c}\right)+r_{M_{c}}(Z \cup c) .
$$

Hence $r_{M_{c}}(Z) \geqslant r_{M_{c}}(Z \cup c)$ and 116.7 holds.

116.8. Neither $a$ nor $b$ has a point on it in either $M$ or $M_{c}$.

Assume there is a point $e$ on $a$ in $M$. Then $M \backslash e$ is 3-connected. Moreover, in $(M \backslash b)^{b}$, we see that $e$ is parallel to $a$ so $(M \backslash b)^{b} \backslash e$, and hence $M \backslash e$, has a c-minor isomorphic to $N$, a contradiction. We conclude that 116.8 holds.

The next step in the proof of Lemma 116 is to show that 116.9. $M_{c} \backslash a, b$ is 2-connected.

Suppose $(A, B)$ is a 1 -separation of $M_{c} \backslash a, b$ having $c$ in $A$. Then

$$
r_{M_{c}}(A)+r_{M_{c}}(B)=r\left(M_{c} \backslash a, b\right)=r\left(M_{c}\right)-1=r(M)-2 .
$$

Thus

$$
\begin{aligned}
r_{M_{c}}(A \cup a) & +r_{M_{c}}(B)-r\left(M_{c}\right) \\
& =r_{M_{c}}(A)+r_{M_{c}}(\{a\})-\sqcap_{M_{c}}(A,\{a\})+r_{M_{c}}(B)-r\left(M_{c}\right) \\
& =\left[r_{M_{c}}(A)+r_{M_{c}}(B)-r\left(M_{c}\right)+1\right]-1+r_{M_{c}}(\{a\})-\sqcap_{M_{c}}(A,\{a\}) \\
& =0-1-2-\sqcap_{M_{c}}(A,\{a\})=1-\sqcap_{M_{c}}(A,\{a\}) .
\end{aligned}
$$


If $\sqcap_{M_{c}}(A,\{a\})=1$, then $(A \cup a \cup b, B)$ is a 1-separation of $M_{c}$ and hence of $M$, a contradiction. We deduce that $\sqcap_{M_{c}}(A,\{a\})=0$ and $(A \cup a \cup b, B)$ is 2-separating in $M_{c}$ and hence in $M$. Thus $B$ consists of a point, say $d$, of $M$. Moreover, $r_{M_{c}}(A \cup a)=r\left(M_{c}\right)$. Thus, as $\sqcap_{M_{c}}(A,\{a\})=0$, we see that

$$
r_{M_{c}}(A)=r\left(M_{c}\right)-2 .
$$

Still working towards proving 116.9, we show next that

116.10. $\{b, d\}$ is a series pair of points in $(M \backslash a)^{b}$.

Recall that $(M \backslash a)^{b}=M_{c} \backslash a \downarrow b$. Now

$$
r_{M_{c}}(\{d, b\})+r_{M_{c}}(A)-r\left(M_{c} \backslash a\right) \leqslant 3+r\left(M_{c}\right)-2-r\left(M_{c}\right)=1 .
$$

Thus $\{d, b\}$ is 2-separating in $M_{c} \backslash a$. It follows that it is also 2-separating in $M_{c} \backslash a \downarrow b$, that is, in $(M \backslash a)^{b}$. But $d$ and $b$ are points in $(M \backslash a)^{b}$, which is 2-connected. We deduce by 116.8 that 116.10 holds.

By 116.10, $(M \backslash a)^{b} / d$, and hence $M / d$, has a c-minor isomorphic to $N$. Next we show that

116.11. $(A-c,\{a, b, c\})$ is a 2-separation of $M \backslash d$.

By $(41), r_{M_{c}}(A-c) \leqslant r\left(M_{c}\right)-2=r(M)-3$ and 116.11 follows.

It follows from 116.11 and Lemma 40 that $M / d$ is 3-connected unless $M$ has a pair $\{e, f\}$ of points such that $e$ and $f$ are parallel in $M / d$. Consider the exceptional case. Then $M$ has $\{d, e, f\}$ as a triangle. Then $\{e, f\} \subseteq A-c$. Thus, by 116.11, $((A-c) \cup d,\{a, b, c\})$ is a 2-separation of $M$, a contradiction. We conclude that 116.9 holds.

By 116.9, we deduce that

116.12. $\lambda_{M_{c} \backslash a, b}\left(X_{a}\right)=1=\lambda_{M_{c} \backslash a}\left(X_{a}\right)$ and $\lambda_{M_{c} \backslash a, b}\left(X_{b}\right)=1=\lambda_{M_{c} \backslash b}\left(X_{b}\right)$.

Since $r\left(M_{c} \backslash a, b\right)=r\left(M_{c} \backslash a\right)-1$, it follows from 116.12 and symmetry that 116.13. $r_{M_{c}}\left(Y_{a}-b\right)=r_{M_{c}}\left(Y_{a}\right)-1$ and $r_{M_{c}}\left(Y_{b}-a\right)=r_{M_{c}}\left(Y_{b}\right)-1$.

It follows from this, symmetry, and the fact that $r_{M}\left(Y_{a} \cup c\right)>r_{M}\left(Y_{a}\right)$ that 116.14. $r_{M}\left(Y_{a}-b\right)=r_{M}\left(Y_{a}\right)-1$ and $r_{M}\left(Y_{b}-a\right)=r_{M}\left(Y_{b}\right)-1$.

By uncrossing,

$$
\begin{aligned}
2 & =\lambda_{M_{c} \backslash a, b}\left(X_{a}\right)+\lambda_{M_{c} \backslash a, b}\left(Y_{b}-a\right) \\
& \geqslant \lambda_{M_{c} \backslash a, b}\left(X_{a} \cap\left(Y_{b}-a\right)\right)+\lambda_{M_{c} \backslash a, b}\left(X_{a} \cup\left(Y_{b}-a\right)\right) .
\end{aligned}
$$

116.15. $X_{a} \cap Y_{b} \neq \emptyset \neq X_{b} \cap Y_{a}$.

Suppose $X_{a} \cap Y_{b}=\emptyset$. Then $Y_{b}-a \subseteq Y_{a}-b$. Thus, by 116.13, $\sqcap_{M_{c}}\left(Y_{a}-b,\{b\}\right)=1=$ $\sqcap_{M_{c}}\left(Y_{a}-b,\{a\}\right)$. Hence, by 116.6, $\sqcap_{M_{c}}\left(Y_{a}-b,\{a, b\}\right)=2$. Thus, by 116.7, $c \in \operatorname{cl}_{M_{c}}\left(Y_{a}-b\right)$. It follows that $\left(Y_{a} \cup c, X_{a}-c\right)$ is 2-separating in $M_{c} \backslash a$. Thus $\left(Y_{a} \cup c \cup a, X_{a}-c\right)$ is 2separating in $M$. As $M$ is 3-connected, we deduce that $X_{a}$ consists of exactly two points, $c$ and $x$, say. If $r_{M_{c}}(\{x, c\})=1$, then, in $M$, we see that $x$ is a point that lies on the line $c$. Thus $M \backslash x$ is 3 -connected. As $(M \backslash a)^{b}$ has a c-minor isomorphic to $N$ and has $x$ 
and $c$ as a parallel pair of points, we deduce that $M \backslash x$ has a c-minor isomorphic to $N$, a contradiction. We conclude that $r_{M_{c}}(\{x, c\})=2$. Thus $\{x\}$ is 1-separating in $M_{c}$, a contradiction. We deduce that $X_{a} \cap Y_{b} \neq \emptyset$ and 116.15 follows by symmetry.

We now choose the non-trivial 2-separation $\left(X_{a}, Y_{a}\right)$ of $M_{c} \backslash a$ such that $\left|X_{a}\right|$ is a minimum subject to the condition that $b \in Y_{a}$. Since $X_{a} \cap Y_{b}$ and $X_{b} \cap Y_{a}$ are both non-empty, we deduce from (42) and symmetry that

$$
\lambda_{M_{c} \backslash a, b}\left(X_{a} \cap Y_{b}\right)=1=\lambda_{M_{c} \backslash a, b}\left(X_{b} \cap Y_{a}\right) .
$$

We show next that

116.16. $\lambda_{M_{c} \backslash a}\left(X_{a} \cap Y_{b}\right)=1=\lambda_{M_{c} \backslash b}\left(X_{b} \cap Y_{a}\right)$.

We have that $1=r_{M_{c}}\left(X_{a} \cap Y_{b}\right)+r_{M_{c}}\left(\left(Y_{a}-b\right) \cup X_{b}\right)-r\left(M_{c} \backslash a, b\right)$. But $r\left(M_{c} \backslash a, b\right)=$ $r\left(M_{c} \backslash a\right)-1$ and, by 116.13, $r_{M_{c}}\left(Y_{a}-b\right)=r_{M_{c}}\left(Y_{a}\right)-1$. Hence $r_{M_{c}}\left(\left(Y_{a}-b\right) \cup X_{b}\right)=$ $r_{M_{c}}\left(Y_{a} \cup X_{b}\right)-1$. Thus 116.16 follows by symmetry.

By the choice of $X_{a}$ and the fact that $b$ and $c$ are the only 2-separating lines of $M \backslash a$, we deduce that $X_{a} \cap Y_{b}$ consists of a single point, say $w$.

116.17. $X_{a}$ consists of a series pair $\{w, c\}$ in $M_{c} \backslash a$.

Suppose $w \notin \operatorname{cl}_{M_{c}}\left(X_{a}-w\right)$. Then $\left(X_{a}-w, Y_{a} \cup w\right)$ violates the choice of $\left(X_{a}, Y_{a}\right)$ unless $\left|X_{a}-w\right|=1$. In the exceptional case, $\{w, c\}$ is a series pair in $M_{c} \backslash a$.

Now suppose that $w \in \mathrm{cl}_{M_{c}}\left(X_{a}-w\right)$. Then $w \in \mathrm{cl}_{M_{c}}\left(X_{b}\right)$. Thus $\left(X_{b} \cup w, Y_{b}-w\right)$ is a 2-separation of $M_{c} \backslash b$. But $Y_{b}-w$ avoids $X_{a}$ so we have a contradiction to 116.15 when we replace $\left(X_{b}, Y_{b}\right)$ by $\left(X_{b} \cup w, Y_{b}-w\right)$ unless $Y_{b}=\{a, w\}$. In the exceptional case, by 116.13, $r\left(Y_{b}\right)=2$ and we have a contradiction to 116.8. We conclude that 116.17 holds.

Since $M_{c} \backslash a$ has $\{w, c\}$ as a series pair, it follows that $M_{c} \backslash a / w$ has a c-minor isomorphic to $N$. Thus so do $(M \backslash a)^{b} / w$ and $M / w$. In $M \backslash a$, we have $\{c, w\}$ and $\{b\}$ as 2-separating sets. Now $w \notin \mathrm{cl}_{M_{c} \backslash a}\left(X_{1}-w\right)$. Hence $r_{M}\left(X_{1}-w\right)=r_{M}\left(X_{1}\right)-1=r(M)-3$. As $r\left(Y_{1}\right)=4$, we deduce that $\left(X_{1}-w, Y_{1}\right)$ is a 2-separation in $M \backslash w$. Thus, by Lemma 40, $M / w$ is 3-connected unless $M$ has a triangle $T$ of points including $w$. In the exceptional case, $T-w \subseteq X_{1}-w$, so $\left(X_{1}, Y_{1}\right)$ is a 2-separation of $M$. This contradiction completes the proof of Lemma 116.

Lemma 117. $\theta \neq 0$.

Proof. Assume that $\theta=0$. Thus $\sqcap\left(X_{1},\{y\}\right)=0$ for all $y$ in $Y_{1}$. We may assume that $\Pi^{*}\left(X_{1},\{y\}\right)=0$ for all $y$ in $Y_{1}$ otherwise, in $M^{*}$, we have $\theta \in\{1,2,3\}$. Thus, for all $y$ in $Y_{1}$, we have $r\left(Y_{1}-y\right)=r\left(Y_{1}\right)=4$. Then, by Lemma 111, none of $M / a, M / b$, nor $M / c$ has a c-minor isomorphic to $N$. Hence we may assume that $a$ and $b$ are deleted to get $N$. But, in $M \backslash a, b$, we see that $\{c\}$ is a component, so $c$ can be contracted to get $N$, a contradiction.

Proof of Lemma 102. By Lemma 101, a minimal non- $N$-3-separator $Y_{1}$ of $M$ having exactly three elements consists of three lines. Above, we looked at the number $\theta$ of members $y$ of $Y_{1}$ for which $\sqcap\left(X_{1},\{y\}\right)=1$. In Lemmas 114 and 115, we showed that $\theta \neq 2$ and $\theta \neq 1$, while Lemmas 116 and 117 showed that $\theta \neq 3$ and $\theta \neq 0$. There are no remaining possibilities for $\theta$, so Lemma 102 holds. 


\section{Finishing the proof of Theorem 57}

By 65, we may now assume that $M$ has a minimal non- $N$-3-separator $Y_{1}$ having at least four elements. As before, we write $X_{1}$ for $E(M)-Y_{1}$. Our next goal is to prove 66 , which we restate here for convenience.

Lemma 118. Let $Y_{1}$ be a minimal non-N-3-separating set having at least four elements. Then $Y_{1}$ contains a doubly labelled element.

Proof. Assume that the lemma fails. For each $e$ in $Y_{1}-E(N)$, let $\nu(e)$ be equal to the unique member of $\left\{\mu(e), \mu^{*}(e)\right\}$ that is defined. Choose $\ell$ in $Y_{1}-E(N)$ to minimize $\nu(\ell)$. By switching to the dual if necessary, we may suppose that $\nu(\ell)=\mu(\ell)$. Let $(A, B)$ be a 2-separation of $M \backslash \ell$ where $A$ is the $N$-side and $|B|=\mu(\ell)$. We now apply Lemma 97 . Part (ii) of that lemma does not hold otherwise, by Lemma 93, $Y_{1}-\ell$ contains a doubly labelled element.

Assume next that (iii) of Lemma 97 holds. Then $\lambda_{M \backslash \ell}\left(Y_{1}-\ell\right)=2$ and $\lambda_{M \backslash \ell}\left(A \cap\left(Y_{1}-\right.\right.$ $\ell))=1=\lambda_{M \backslash \ell}\left(B \cap\left(Y_{1}-\ell\right)\right)$, while $\lambda_{M \backslash \ell}\left(A \cap X_{1}\right)=2=\lambda_{M \backslash \ell}\left(B \cap X_{1}\right)$. Then using the partitions $\left(A \cap\left(Y_{1}-\ell\right), A \cap X_{1}, B\right)$ and $\left(B \cap\left(Y_{1}-\ell\right), B \cap X_{1}, A\right)$ as $(A, B, C)$ in Lemma 34, we deduce that $\sqcap\left(A \cap\left(Y_{1}-\ell\right), A \cap X_{1}\right)=1$ and $\sqcap\left(B \cap\left(Y_{1}-\ell\right), B \cap X_{1}\right)=1$.

Now $M \backslash \ell$ is the 2-sum of 2-polymatroids $M_{A}$ and $M_{B}$ having ground sets $A \cup q$ and $B \cup q$, respectively. Since $M \backslash \ell$ is 2 -connected, it follows by Proposition 47, that each of $M_{A}$ and $M_{B}$ is 2-connected. Now $\lambda_{M \backslash \ell}\left(B \cap\left(Y_{1}-\ell\right)\right)=\sqcap_{M \backslash \ell}\left(B \cap\left(Y_{1}-\ell\right),\left(B \cap X_{1}\right) \cup A\right)=1$ and $\sqcap_{M_{B}}\left(B \cap\left(Y_{1}-\ell\right), B \cap X_{1}\right)=1$. Noting that $M \backslash \ell=P\left(M_{A}, M_{B}\right) \backslash q$, we see that, in $P\left(M_{A}, M_{B}\right)$, we have $\sqcap\left(B \cap\left(Y_{1}-\ell\right),\left(B \cap X_{1}\right) \cup A \cup q\right)=1$. Hence $\sqcap_{M_{B}}\left(B \cap\left(Y_{1}-\ell\right),(B \cap\right.$ $\left.\left.X_{1}\right) \cup q\right)=1$. Thus $M_{B}$ is the 2-sum of two 2-connected 2-polymatroids $M_{B, Y}$ and $M_{B, X}$ having ground sets $\left(B \cap\left(Y_{1}-\ell\right)\right) \cup s$ and $\left(B \cap X_{1}\right) \cup q \cup s$. Note that $M_{B}=P\left(M_{B, X}, M_{B, Y}\right) \backslash s$. Let $M_{B}^{\prime}=P\left(M_{B, X}, M_{B, Y}\right)$ and consider $P\left(M_{A}, M_{B}^{\prime}\right)$ noting that deleting $q$ and $s$ from this 2-polymatroid gives $M \backslash \ell$.

By Lemma 31(ii),

$$
\sqcap(A, B)+\sqcap\left(B \cap X_{1}, B \cap\left(Y_{1}-\ell\right)\right)=\sqcap\left(A \cup\left(B \cap X_{1}\right), B \cap\left(Y_{1}-\ell\right)\right)+\sqcap\left(A, B \cap X_{1}\right) .
$$

Since the first three terms in this equation equal one,

$$
\sqcap\left(A, B \cap X_{1}\right)=1 .
$$

We deduce, by Lemma 51(i) that if $y \in B \cap\left(Y_{1}-\ell\right)$, then $M \backslash \ell \backslash y$ has a special $N$-minor.

Now $M_{B, X}$ has $q$ and $s$ as points. We show next that

118.1. $\lambda_{M_{B, X} / s}(\{q\})=0$.

Assume that $\lambda_{M_{B, X} / s}(\{q\}) \neq 0$. When we contract $s$ in $M_{B}^{\prime}$, the set $B \cap\left(Y_{1}-\ell\right)$ becomes 1-separating. Moreover, in $M_{B}^{\prime} /\left(B \cap\left(Y_{1}-\ell\right)\right)$, the element $s$ is a loop, so $M_{B}^{\prime} \backslash s /\left(B \cap\left(Y_{1}-\ell\right)\right)=M_{B}^{\prime} / s /\left(B \cap\left(Y_{1}-\ell\right)\right)$. It follows that $\sqcap_{M \backslash \ell /\left(B \cap\left(Y_{1}-\ell\right)\right.}\left(A, B \cap X_{1}\right)=1$. Hence, by Lemma 51(ii), if $y \in B \cap\left(Y_{1}-\ell\right)$, then $M \backslash \ell / y$ has a special $N$-minor. Thus each $y$ in $B \cap\left(Y_{1}-\ell\right)$ is doubly labelled. This contradiction completes the proof of 118.1 . 
By 118.1, $\{q, s\}$ is a parallel pair of points in $M_{B, X}$. From considering $P\left(M_{A}, M_{B}^{\prime}\right)$, we deduce that $\lambda_{M \backslash \ell}\left(B \cap X_{1}\right)=1$. This contradiction implies that (iii) of Lemma 97 does not hold.

It remains to consider when (i) of Lemma 97 holds, that is, $\left(X_{1}, Y_{1}-\ell\right)$ is a 2-separation of $M \backslash \ell$. We now apply Lemma 91. By assumption, (i) of that lemma does not hold; if (iii) holds, then $\mu(y)<\mu(\ell)$, contradicting the choice of $\ell$. Thus (ii) of Lemma 91 holds, that is, $\sqcap\left(\{y\}, X_{1}\right) \neq 1$ for some $y$ in $Y_{1}-\ell$. Then, by Lemma $90, \sqcap\left(Y_{1}-y, X_{1}\right)=0$ for all $y$ in $Y_{1}-\ell$. Thus, by Lemma 92 and the choice of $\ell$, (iii)(b) rather than (iii)(a) of that lemma holds. Then, with $z$ as in (iii)(b), $\left(X_{1}-z,\left(Y_{1}-\ell\right) \cup z\right)$ is a 2-separation of $M \backslash \ell$ having $X_{1}-z$ as the $N$-side. Since $z$ is a point, we have a contradiction to Lemma 95.

The doubly labelled element found in the last lemma will be crucial in completing the proof of Theorem 57. We shall need another preliminary lemma.

Lemma 119. Let $\ell$ be a doubly labelled element of $M$. Then $M \backslash \ell$ does not have a series pair of points $\{a, b\}$ such that $r(\{a, b, \ell\})=3$.

Proof. Assume that $M \backslash \ell$ does have such a series pair $\{a, b\}$. By Lemma $58, \ell$ is a line. Thus $M / \ell$ has $\{a, b\}$ as a parallel pair of points or has $a$ or $b$ as a loop. In each case, $M$ has $a$ or $b$ as a doubly labelled point, a contradiction.

We will now take $\ell$ to be a doubly labelled element of $Y_{1}$, a minimal non- $N$-3-separating set having at least four elements.

Lemma 120. There is a 2-separating set $Q$ in $M \backslash \ell$ such that $Q \subseteq Y_{1}-\ell$ and $|Q| \geqslant 2$ and contains no points.

Proof. Suppose $\ell \notin \operatorname{cl}\left(Y_{1}-\ell\right)$. Then $\left(X_{1}, Y_{1}-\ell\right)$ is a 2-separation of $M \backslash \ell$ and $r\left(Y_{1}\right)=$ $r\left(Y_{1}-\ell\right)+1$. Then, by Lemma 119, $Y_{1}-\ell$ does not consist of a series pair of points. Hence, by Lemma 95, $Y_{1}-\ell$ contains no points so the result holds by taking $Q=Y_{1}-\ell$.

We may now assume that $\ell \in \operatorname{cl}\left(Y_{1}-\ell\right)$. Let $(A, B)$ be a 2-separation of $M \backslash \ell$ where $A$ is the $N$-side and $|B|=\mu(\ell)$. Since $|B| \geqslant 3$, it follows by Lemma 95 that $B$ contains no points. If $B \subseteq Y_{1}-\ell$, then the lemma holds by taking $Q=B$. Thus we may assume that $B \cap X_{1} \neq \emptyset$.

Since $X_{1}$ and $A$ are the $N$-sides of their respective separations and $|E(N)| \geqslant 4$, we see that $\left|A \cap X_{1}\right| \geqslant 2$. If $A \subseteq X_{1}$, then $B \supseteq Y_{1}-\ell$, so $(A, B \cup \ell)$ is a 2-separation of $M$, a contradiction. Likewise, if $B \subseteq X_{1}$, then $(A \cup \ell, B)$ is a 2-separation of $M$, a contradiction. We conclude that $(A, B)$ crosses $\left(X_{1}, Y_{1}-\ell\right)$.

Since $\left|A \cap X_{1}\right| \geqslant 2$ and $\ell \in \operatorname{cl}\left(Y_{1}-\ell\right)$, it follows that $\lambda_{M \backslash \ell}\left(A \cap X_{1}\right) \geqslant 2$ otherwise $\left(A \cap X_{1}, B \cup Y_{1}\right)$ is a 2 -separation of $M$, a contradiction. Then, by uncrossing, we deduce that $\lambda_{M \backslash \ell}\left(B \cap Y_{1}\right) \leqslant 1$. Since $B$ contains no points, the lemma holds with $Q=B \cap Y_{1}$ unless this set contains a single line.

Consider the exceptional case. As $|B| \geqslant 3$, we deduce that $\left|B \cap X_{1}\right| \geqslant 2$. Now $\lambda_{M \backslash \ell}(B \cap$ $\left.X_{1}\right) \geqslant 2$ otherwise, as $\ell \in \operatorname{cl}\left(Y_{1}-\ell\right)$, we obtain the contradiction that $\left(B \cap X_{1}, A \cup Y_{1}\right)$ is a 2-separation of $M$. Hence, by uncrossing, $\lambda_{M \backslash \ell}\left(A \cap Y_{1}\right)=1$ and, as $\left|Y_{1}\right| \geqslant 4$, it follows using Lemma 95 that the lemma holds by taking $Q=A \cap Y_{1}$. 
Lemma 121. The 2-polymatroid $M \backslash \ell$ has a 2-separation $\left(D_{1}, D_{2}\right)$ where $D_{2}$ has at least two elements, is contained in $Y_{1}-\ell$, and contains no points. Moreover, either

(i) $D_{2} \cup \ell=Y_{1}$; and $\sqcap\left(D_{1},\{\ell\}\right)=0$ and $\sqcap\left(D_{2},\{\ell\}\right)=1$; or

(ii) $Y_{1}-\ell-D_{2} \neq \emptyset$ and $\sqcap\left(D_{1},\{\ell\}\right)=0=\sqcap\left(D_{2},\{\ell\}\right)$

Proof. Let $D_{2}$ be the set $Q$ found in Lemma 120 and let $D_{1}=E(M \backslash \ell)-D_{2}$. Now $\left(D_{1}, D_{2}\right)$ is a 2 -separation of $M \backslash \ell$. Thus, there are the following four possibilities.

(I) $\sqcap\left(D_{1},\{\ell\}\right)=1=\sqcap\left(D_{2},\{\ell\}\right)$;

(II) $\sqcap\left(D_{1},\{\ell\}\right)=1$ and $\sqcap\left(D_{2},\{\ell\}\right)=0$;

(III) $\sqcap\left(D_{1},\{\ell\}\right)=0$ and $\sqcap\left(D_{2},\{\ell\}\right)=1$; and

(IV) $\sqcap\left(D_{1},\{\ell\}\right)=0=\sqcap\left(D_{2},\{\ell\}\right)$.

121.1. Neither (I) nor (II) holds.

Suppose (I) or (II) holds. Then $\lambda_{M}\left(D_{1} \cup \ell\right)=2$, so $\lambda_{M}\left(D_{2}\right)=2$ and $\left|D_{2}\right| \geqslant 2$. Since $D_{2}$ contains no points and $D_{2} \subseteq Y_{1}-\ell$, we get a contradiction to the minimality of $Y_{1}$. Thus 121.1 holds.

121.2. If (III) holds, then $D_{2} \cup \ell=Y_{1}$.

As $\lambda_{M}\left(D_{2} \cup \ell\right)=2$, we must have that $D_{1} \cap Y_{1}=\emptyset$ otherwise $D_{2} \cup \ell$ violates the minimality of $Y_{1}$. Thus 121.2 holds.

121.3. If (IV) holds, then $D_{2} \cup \ell=Y_{1}$.

Suppose $D_{1} \cap Y_{1}=\emptyset$. Then $D_{1}=X_{1}$ and $D_{2}=Y_{1}-\ell$. Thus $\lambda_{M}\left(X_{1}\right)>2$ as $\sqcap\left(D_{2},\{\ell\}\right)=0$. This contradiction establishes that 121.3 holds and thereby completes the proof of the lemma.

Lemma 122. The 2-polymatroid $M / \ell$ has a 2-separation $\left(C_{1}, C_{2}\right)$ where $C_{2}$ contains at least two elements and is contained in $Y_{1}-\ell$, and contains no points of $M$. Moreover, either

(i) $C_{2} \cup \ell=Y_{1}$; and $\sqcap\left(C_{1},\{\ell\}\right)=1$ and $\sqcap\left(C_{2},\{\ell\}\right)=2$; or

(ii) $Y_{1}-\ell-C_{2} \neq \emptyset$ and $\sqcap\left(C_{1},\{\ell\}\right)=2=\sqcap\left(C_{2},\{\ell\}\right)$.

Proof. We apply the preceding lemma to $M^{*} \backslash \ell$ recalling that $\left(M^{*} \backslash \ell\right)^{b}=(M / \ell)^{*}$ and that the connectivity functions of $M^{*} \backslash \ell$ and $M / \ell$ are equal. Thus $M / \ell$ does indeed have a 2-separation $\left(C_{1}, C_{2}\right)$ where $C_{2}$ contains at least two elements, is contained in $Y_{1}-\ell$, and contains no points of $M^{*}$. Thus $C_{2}$ contains no points of $M$. Since $r(E-\ell)=r(M)$, one easily checks that $\Pi^{*}\left(C_{i},\{\ell\}\right)+\sqcap\left(C_{j},\{\ell\}\right)=2$ where $\{i, j\}=\{1,2\}$. The lemma now follows from the preceding one. 
Lemma 123. There are 2-separations $\left(D_{1}, D_{2}\right)$ and $\left(C_{1}, C_{2}\right)$ of $M \backslash \ell$ and $M / \ell$, respectively, such that each of $D_{2}$ and $C_{2}$ contains at least two elements, both $D_{2}$ and $C_{2}$ are contained in $Y_{1}-\ell$, and neither $D_{2}$ nor $C_{2}$ contains any points of $M$. Moreover, $Y_{1}-\ell-D_{2} \neq$ $\emptyset \neq Y_{1}-\ell-C_{2}$ and $\sqcap\left(D_{1},\{\ell\}\right)=0=\sqcap\left(D_{2},\{\ell\}\right)$ while $\sqcap\left(C_{1},\{\ell\}\right)=2=\sqcap\left(C_{2},\{\ell\}\right)$.

Proof. Assume that (i) of Lemma 121 holds. Then, as $D_{2}=Y_{1}-\ell$, we see that $\sqcap\left(Y_{1}-\right.$ $\ell,\{\ell\})=1$. Thus (i) of Lemma 122 cannot hold. Moreover, if (ii) of Lemma 122 holds, then $\sqcap\left(C_{2},\{\ell\}\right)=2$. This is a contradiction as $\sqcap\left(D_{2},\{\ell\}\right)=1$ and $C_{2} \subseteq D_{2}$. We conclude that (ii) of Lemma 121 holds. If (i) of Lemma 122 holds, then $\sqcap\left(X_{1},\{\ell\}\right)=1$. But $X_{1} \subseteq D_{1}$ and $\sqcap\left(D_{1},\{\ell\}\right)=0$, a contradiction.

We now use the 2-separations $\left(D_{1}, D_{2}\right)$ and $\left(C_{1}, C_{2}\right)$ of $M \backslash \ell$ and $M / \ell$, respectively, found in the last lemma.

Lemma 124. The partitions $\left(D_{1}, D_{2}\right)$ and $\left(C_{1}, C_{2}\right)$ have the following properties.

(i) $\lambda_{M \backslash \ell}\left(C_{1}\right)=3=\lambda_{M \backslash \ell}\left(C_{2}\right)$;

(ii) $\left(D_{1}, D_{2}\right)$ and $\left(C_{1}, C_{2}\right)$ cross;

(iii) each of $D_{1} \cap C_{2}, D_{2} \cap C_{2}$, and $D_{2} \cap C_{1}$ consists of a single line, and $C_{2} \cup D_{2}=Y_{1}-\ell$; and

(iv) $\lambda_{M \backslash \ell}\left(D_{1} \cap C_{1}\right)=\lambda_{M}\left(D_{1} \cap C_{1}\right)=2$.

Proof. We have $\sqcap\left(D_{1},\{\ell\}\right)=0=\sqcap\left(D_{2},\{\ell\}\right)$ and $\sqcap\left(C_{1},\{\ell\}\right)=2=\sqcap\left(C_{2},\{\ell\}\right)$. Thus neither $C_{1}$ nor $C_{2}$ is contained in $D_{1}$ or $D_{2}$, so (ii) holds. Moreover, as $r\left(C_{1} \cup \ell\right)+$ $r\left(C_{2} \cup \ell\right)-r(M)=3$, we see that (i) holds. To prove (iii) and (iv), we use an uncrossing argument. We have, for each $i$ in $\{1,2\}$,

$$
\begin{aligned}
1+3 & =\lambda_{M \backslash \ell}\left(D_{2}\right)+\lambda_{M \backslash \ell}\left(C_{i}\right) \\
& \geqslant \lambda_{M \backslash \ell}\left(D_{2} \cap C_{i}\right)+\lambda_{M \backslash \ell}\left(D_{2} \cup C_{i}\right) .
\end{aligned}
$$

Since $D_{2} \cap C_{i} \neq \emptyset$ and contains no points and $\ell \in \operatorname{cl}\left(C_{j}\right)$ where $j \neq i$, we deduce that $\lambda_{M \backslash \ell}\left(D_{2} \cap C_{i}\right)=\lambda_{M}\left(D_{2} \cap C_{i}\right) \geqslant 2$. Thus $\lambda_{M \backslash \ell}\left(D_{2} \cup C_{i}\right) \leqslant 2$. Hence, as $\ell \in \operatorname{cl}\left(C_{i}\right)$, we see that

$$
2 \geqslant \lambda_{M \backslash \ell}\left(D_{2} \cup C_{i}\right)=\lambda_{M}\left(D_{2} \cup C_{i} \cup \ell\right) .
$$

But $D_{2} \cup C_{2} \subseteq Y_{1}-\ell$, so, by the definition of $Y_{1}$, we deduce that

$$
D_{2} \cup C_{2}=Y_{1}-\ell \text { and } D_{1} \cap C_{1}=X_{1} .
$$

Moreover, as $\lambda_{M \backslash \ell}\left(D_{2} \cup C_{i}\right)=2$, we see that $\lambda_{M \backslash \ell}\left(D_{2} \cap C_{i}\right)=2$. Hence

$$
\lambda_{M}\left(D_{2} \cap C_{i}\right)=2 .
$$

Since $D_{1} \cap C_{1}=X_{1}$ and $D_{1} \cap C_{2}$ is non-empty containing no points, it follows from (44) that

$$
2=\lambda_{M \backslash \ell}\left(D_{2} \cup C_{i}\right)=\lambda_{M}\left(D_{2} \cup C_{i} \cup \ell\right)=\lambda_{M \backslash \ell}\left(D_{1} \cap C_{j}\right)=\lambda_{M}\left(D_{1} \cap C_{j}\right) .
$$

Thus (iv) holds. By that and (46), it follows, using the minimality of $Y_{1}$, that each of $D_{1} \cap C_{2}, D_{2} \cap C_{2}$, and $D_{2} \cap C_{1}$ consist of a single line in $M$. Hence (iii) holds. 
For each $(i, j)$ in $\{(1,2),(2,2),(2,1)\}$, let $C_{i} \cap D_{j}=\left\{\ell_{i j}\right\}$.

Lemma 125. The following hold.

(i) $r\left(D_{2}\right)=3$ so $r\left(D_{1}\right)=r(M)-2$;

(ii) $r\left(D_{1}\right)=r\left(X_{1}\right)+1$;

(iii) $r\left(Y_{1}\right)=5$; and

(iv) $r\left(C_{2}\right)=4$.

Proof. Now $D_{2}$ consists of two lines, $\ell_{12}$ and $\ell_{22}$. Suppose first that $r\left(D_{2}\right)=2$. Then both $M \backslash \ell_{12}$ and $M \backslash \ell_{22}$ are 3-connected. Without loss of generality, $M / \ell_{12}$ has $N$ as a c-minor. But $M / \ell_{12}$ has $\ell_{22}$ as a loop, so $M \backslash \ell_{22}$ is 3-connected having a c-minor isomorphic to $N$. Thus $r\left(D_{2}\right) \geqslant 3$.

Now suppose that $r\left(D_{2}\right)=4$. Then $r\left(D_{1}\right)=r(M)-3$. Clearly $r\left(D_{1} \cup \ell_{22}\right) \leqslant r(M)-1$. Now $D_{1} \cup \ell_{22} \supseteq C_{2}$ so $r\left(D_{1} \cup \ell_{22} \cup \ell\right) \leqslant r(M)-1$. Hence $\left\{\ell_{12}\right\}$ is 2-separating in $M$, a contradiction. Hence (i) holds.

Since $C_{2} \cup D_{2}=Y_{1}-\ell$, we see that $D_{1}=X_{1} \cup \ell_{21}$. Suppose $r\left(D_{1}\right)=r\left(X_{1}\right)$. As $X_{1} \subseteq C_{1}$, we deduce that $\ell_{21} \in \operatorname{cl}\left(C_{1}\right)$. But $\ell \in \operatorname{cl}\left(C_{1}\right)$. Hence

$$
\begin{aligned}
r(M)+3 & =r\left(C_{1}\right)+r\left(C_{2}\right) \\
& =r\left(C_{1} \cup \ell\right)+r\left(C_{2} \cup \ell\right) \\
& =r\left(C_{1} \cup \ell \cup \ell_{21}\right)+r\left(C_{2} \cup \ell\right) \\
& \geqslant r\left(C_{1} \cup C_{2} \cup \ell\right)+r\left(\left\{\ell, \ell_{21}\right\}\right) .
\end{aligned}
$$

Thus $r\left(\left\{\ell, \ell_{21}\right\}\right) \leqslant 3$, so $\sqcap\left(D_{1},\{\ell\}\right) \geqslant 1$, a contradiction. Hence $r\left(D_{1}\right) \geqslant r\left(X_{1}\right)+1$.

Suppose $r\left(D_{1}\right)=r\left(X_{1}\right)+2$. Then

$$
r(M)+1=r\left(D_{1}\right)+r\left(D_{2}\right)=r\left(X_{1}\right)+2+3,
$$

so $r\left(X_{1}\right)=r(M)-4$. Thus $r\left(Y_{1}\right)=6$. Now $r\left(C_{2}\right)=r\left(C_{2} \cup \ell\right)$. Thus $6=r\left(Y_{1}\right)=r\left(Y_{1}-\ell\right)$. Since $Y_{1}-\ell$ consists of three lines, two of which are in $D_{2}$, we deduce that $r\left(D_{2}\right)=4$, a contradiction to (i). We conclude that $r\left(D_{1}\right)=r\left(X_{1}\right)+1$, that is, (ii) holds.

Finally, as $r\left(D_{2}\right)=3$, we see that $r(M)=r\left(D_{1}\right)+r\left(D_{2}\right)-1=\left[r\left(X_{1}\right)+1\right]+3-1$. But $r(M)=r\left(X_{1}\right)+r\left(Y_{1}\right)-2$. Thus $r\left(Y_{1}\right)=5$, so (iii) holds. Moreover, $r\left(Y_{1}-\ell\right)=5$, that is, $r\left(C_{2} \cup D_{2}\right)=5$. Now $C_{2}$ consists of two lines so $r\left(C_{2}\right) \leqslant 4$. Thus

$$
\begin{aligned}
4+3 & \geqslant r\left(C_{2}\right)+3 \\
& =r\left(C_{2}\right)+r\left(D_{2}\right) \\
& \geqslant r\left(C_{2} \cup D_{2}\right)+r\left(C_{2} \cap D_{2}\right) \\
& =5+r\left(\left\{\ell_{22}\right\}\right) \\
& =5+2 .
\end{aligned}
$$

We deduce that $r\left(C_{2}\right)=4$ so (iv) holds. 
By proving the following lemma, we will establish the final contradiction that completes the proof of Theorem 57 .

Lemma 126. The 2-polymatroid $M / \ell_{22}$ is 3-connected having a c-minor isomorphic to $N$.

Proof. First we show the following.

126.1. $\sqcap\left(D_{1},\left\{\ell_{i 2}\right\}\right)=0$ for each $i$ in $\{1,2\}$.

Suppose $\sqcap\left(D_{1},\left\{\ell_{i 2}\right\}\right) \geqslant 1$. Then $r\left(D_{1} \cup \ell_{i 2}\right) \leqslant r\left(D_{1}\right)+1$. But $\ell \in \operatorname{cl}\left(C_{i}\right) \subseteq \operatorname{cl}\left(D_{1} \cup \ell_{i 2}\right)$, so $r\left(D_{1} \cup \ell \cup \ell_{i 2}\right) \leqslant r\left(D_{1}\right)+1$. Also $r\left(\left\{\ell_{j 2}\right\}\right)=2$ where $\{i, j\}=\{1,2\}$. Thus

$$
\begin{aligned}
r\left(D_{1} \cup \ell \cup \ell_{i 2}\right)+r\left(\left\{\ell_{j 2}\right\}\right) & \leqslant r\left(D_{1}\right)+1+2 \\
& =r(M)-2+1+2 \\
& =r(M)+1 .
\end{aligned}
$$

Hence $\left\{\ell_{j 2}\right\}$ is 2-separating in $M$, a contradiction. Hence 126.1 holds.

Now $M \backslash \ell$ has a c-minor isomorphic to $N$ and $\sqcap\left(D_{1}, D_{2}\right)=1$. As $\sqcap\left(D_{1},\left\{\ell_{i 2}\right\}\right)=0$, Lemma 29 implies that $\Pi_{M / \ell_{i 2}}\left(D_{1}, D_{2}-\ell_{i 2}\right)=1$ for each $i$ in $\{1,2\}$. Thus, by Lemma 51(ii), 126.2. $M \backslash \ell / \ell_{i 2}$ has a c-minor isomorphic to $N$ for each $i$ in $\{1,2\}$.

It remains to show that $M / \ell_{22}$ is 3 -connected. By Lemma 59 , this polymatroid is certainly 2-connected. Next we show that

126.3. $\ell$ and $\ell_{21}$ are parallel lines in $M / \ell_{22}$.

To see this, note that, by Lemma 125(iv),

$$
r\left(C_{2} \cup \ell\right)=r\left(C_{2}\right)=r\left(\left\{\ell_{21}, \ell_{22}\right\}\right) .
$$

Also, for each $i$ in $\{1,2\}$, we have $\sqcap\left(\{\ell\},\left\{\ell_{2 i}\right\}\right) \leqslant \sqcap\left(\{\ell\}, D_{i}\right)=0$, so 126.3 holds.

Now take a fixed c-minor of $M \backslash \ell / \ell_{22}$ isomorphic to $N$; call it $N_{1}$. Let $\left(A^{\prime} \cup \ell, B^{\prime}\right)$ be a 2 -separation of $M / \ell_{22}$ in which the non- $N_{1}$-side has maximum size and $\ell \notin A^{\prime}$. By Lemma 88, both $A^{\prime} \cup \ell$ and $B^{\prime}$ have at least three elements.

126.4. $\ell_{21} \in A^{\prime}$.

To see this, note that, since $\ell$ and $\ell_{21}$ are parallel lines in $M / \ell_{22}$, if $\ell_{21} \in B^{\prime}$, then $\ell \in \mathrm{cl}_{M / \ell_{22}}\left(B^{\prime}\right)$, so $\sqcap_{M / \ell_{22}}\left(A^{\prime} \cup \ell, B^{\prime}\right) \geqslant 2$, a contradiction.

126.5. $r_{M}\left(D_{1} \cap C_{1}\right)=r(M)-3=r_{M / \ell_{22}}\left(D_{1} \cap C_{1}\right)$.

By Lemma 124(iii), $D_{1} \cap C_{1}=X_{1}$. By Lemma 125(i) and (ii), $r\left(D_{1}\right)=r(M)-2$ and $r\left(D_{1}\right)=r\left(D_{1} \cap C_{1}\right)+1$, so $r_{M}\left(D_{1} \cap C_{1}\right)=r(M)-3$. By 126.1, $\sqcap\left(D_{1} \cap C_{1},\left\{\ell_{22}\right\}\right)=0$, so $r_{M}\left(D_{1} \cap C_{1}\right)=r_{M / \ell_{22}}\left(D_{1} \cap C_{1}\right)$.

126.6. $r_{M / \ell_{22}}\left(\left(D_{1} \cap C_{1}\right) \cup \ell_{12}\right)=r(M)-2$. 
To see this, observe that

$$
\begin{aligned}
r_{M / \ell_{22}}\left(\left(D_{1} \cap C_{1}\right) \cup \ell_{12}\right) & =r\left(\left(D_{1} \cap C_{1}\right) \cup \ell_{12} \cup \ell_{22}\right)-2 \\
& =r\left(\left(D_{1} \cap C_{1}\right) \cup \ell_{12} \cup \ell \cup \ell_{22}\right)-2 \text { as } \ell \in \operatorname{cl}\left(C_{1}\right) ; \\
& =r\left(M \backslash \ell_{21}\right)-2 \\
& =r(M)-2 .
\end{aligned}
$$

By combining 126.5 and 126.6, we deduce that 126.7. $r_{M / \ell_{22}}\left(D_{1} \cap C_{1}\right)=r_{M / \ell_{22}}\left(\left(D_{1} \cap C_{1}\right) \cup \ell_{12}\right)-1$.

Next we show that 126.8. $\lambda_{M / \ell_{22}}\left(\left\{\ell_{12}, \ell_{21}, \ell\right\}\right)=1$ or $\lambda_{M / \ell_{22}}\left(\left\{\ell_{21}, \ell\right\}\right)=1$.

Recall that $X_{1}=D_{1} \cap C_{1}$ and $Y_{1}=\left\{\ell, \ell_{12}, \ell_{21}, \ell_{22}\right\}$. By uncrossing, we have

$$
\begin{aligned}
1+2 & =\lambda_{M / \ell_{22}}\left(B^{\prime}\right)+\lambda_{M / \ell_{22}}\left(X_{1}\right) \\
& \geqslant \lambda_{M / \ell_{22}}\left(B^{\prime} \cup X_{1}\right)+\lambda_{M / \ell_{22}}\left(B^{\prime} \cap X_{1}\right) .
\end{aligned}
$$

As $\left|B^{\prime}\right| \geqslant 3$, it follows by 126.4 that $\left|B^{\prime} \cap X_{1}\right| \geqslant 2$. Suppose $\lambda_{M / \ell_{22}}\left(B^{\prime} \cap X_{1}\right)=1$. Now $B^{\prime} \cap X_{1} \subseteq D_{1}$, so $\sqcap\left(B^{\prime} \cap X_{1},\left\{\ell_{22}\right\}\right) \leqslant \sqcap\left(D_{1},\left\{\ell_{22}\right\}\right) \leqslant 0$. Thus, by Lemma $29, \lambda_{M}\left(B^{\prime} \cap X_{1}\right)=$ 1. This contradiction implies that $\lambda_{M / \ell_{22}}\left(B^{\prime} \cap X_{1}\right)=2$, so $1=\lambda_{M / \ell_{22}}\left(B^{\prime} \cup X_{1}\right)$. Now, by $126.4, \ell_{21} \in A^{\prime}$, so $E-\ell_{22}-\left(B^{\prime} \cup X_{1}\right)$ is $\left\{\ell_{12}, \ell_{21}, \ell\right\}$ or $\left\{\ell_{21}, \ell\right\}$. Thus 126.8 holds.

Suppose $\lambda_{M / \ell_{22}}\left(\left\{\ell_{12}, \ell_{21}, \ell\right\}\right)=1$. Then, as $\ell_{22}$ is skew to $X_{1}$ in $M$, we deduce that $\lambda_{M}\left(\left\{\ell_{12}, \ell_{21}, \ell, \ell_{22}\right\}\right)=1$, that is, $\lambda_{M}\left(Y_{1}\right)=1$, a contradiction. We conclude that $\lambda_{M / \ell_{22}}\left(\left\{\ell_{21}, \ell\right\}\right)=1$.

By Lemma 29 , as $\lambda_{M}\left(D_{1} \cap C_{1}\right)=2$ and $\sqcap\left(D_{1} \cap C_{1},\left\{\ell_{22}\right\}\right)=0$, we see that $\lambda_{M / \ell_{22}}\left(D_{1} \cap\right.$ $\left.C_{1}\right)=2$. Thus, by 126.7 and Lemma 125 ,

$$
\begin{aligned}
2 & =r_{M / \ell_{22}}\left(D_{1} \cap C_{1}\right)+r_{M / \ell_{22}}\left(\left\{\ell_{12}, \ell_{21}, \ell\right\}\right)-r\left(M / \ell_{22}\right) \\
& =\left[r_{M / \ell_{22}}\left(D_{1} \cap C_{1}\right)+1\right]+\left[r_{M / \ell_{22}}\left(\left\{\ell_{12}, \ell_{21}, \ell\right\}\right)-1\right]-r\left(M / \ell_{22}\right) \\
& =r_{M / \ell_{22}}\left(\left(D_{1} \cap C_{1}\right) \cup \ell_{12}\right)+\left[r\left(Y_{1}\right)-2-1\right]-r\left(M / \ell_{22}\right) \\
& =r_{M / \ell_{22}}\left(\left(D_{1} \cap C_{1}\right) \cup \ell_{12}\right)+\left[r\left(C_{2}\right)-2\right]-r\left(M / \ell_{22}\right) \\
& =r_{M / \ell_{22}}\left(\left(D_{1} \cap C_{1}\right) \cup \ell_{12}\right)+r_{M / \ell_{22}}\left(\left\{\ell_{21}, \ell\right\}\right)-r\left(M / \ell_{22}\right) \\
& =\lambda_{M / \ell_{22}}\left(\left\{\ell_{21}, \ell\right\}\right) .
\end{aligned}
$$

This contradiction to 126.8 completes the proof of the lemma and thereby finishes the proof of Theorem 57 .

\section{Acknowledgements}

The authors thank the referee for reading the paper very carefully and correcting a number of errors. The second and third authors were supported by the New Zealand Marsden Fund. 


\section{References}

[1] B. D. Beavers. Circuits and Structure in Matroids and Graphs, Ph.D. dissertation, Louisiana State University, 2006.

[2] R. E. Bixby. A simple theorem on 3-connectivity. Linear Algebra Appl., 45:123-126, 1982.

[3] J. Geelen, B. Gerards, and G. Whittle. Matroid T-connectivity. SIAM J. Discrete Math., 20:588-596, 2006.

[4] J. Geelen, B. Gerards, and G. Whittle. Solving Rota's Conjecture. Notices Amer. Math. Soc., 61:736-743, 2014.

[5] D. Hall. Essential elements in connected k-polymatroids. Adv. in App. Math., 50:281291, 2013.

[6] T. Helgason. Aspects of the theory of hypermatroids. In Hypergraph Seminar (eds. C. Berge and D. K. Ray-Chaudhuri), volume 411 of Lecture Notes in Math., pages 191-214, Springer-Verlag, Berlin, 1974.

[7] S. Jowett. Recognition Problems for Connectivity Functions, M.Sc. thesis, Victoria University of Wellington, 2015.

[8] S. Jowett, S. Mo, and G. Whittle. Connectivity functions and polymatroids. Adv. in Appl. Math., 81:1-12, 2016.

[9] L. Lovász. Matroids and geometric graphs. In Combinatorial Surveys: Proc. Sixth British Combinatorial Conference (ed. P. J. Cameron), pages 45-86, Academic Press, London, 1977.

[10] L. Lovász and M. D. Plummer. Matching Theory, North-Holland, Amsterdam, 1986.

[11] F. Matúš. Adhesivity of polymatroids. Discrete Math., 307:2464-2477, 2007.

[12] C. J. H. McDiarmid. Rado's Theorem for polymatroids. Math. Proc. Camb. Phil. Soc., 78:263-281, 1975.

[13] S. Mo. The Structure of Connectivity Functions, M.Sc. thesis, Victoria University of Wellington, 2014.

[14] J. Oxley. Matroid Theory, Second edition, Oxford University Press, New York, 2011.

[15] J. Oxley, C. Semple, and G. Whittle. The structure of the 3-separations of 3connected matroids. J. Combinatorial Theory, Ser. B, 92:257-293, 2004.

[16] J. Oxley, C. Semple, and G. Whittle. A Wheels-and-Whirls Theorem for 3-connected 2-polymatroids. SIAM J. Discrete Math., 30:493-524, 2016.

[17] J. Oxley and G. Whittle. Tutte invariants of 2-polymatroids. J. Combinatorial Theory, Ser. B, 59:210-244, 1991.

[18] J. Oxley and G. Whittle. Connectivity of submodular functions. Discrete Math., 105:173-184, 1992.

[19] P. D. Seymour. Decomposition of regular matroids. J. Combinatorial Theory, Ser. $B, 28: 305-359,1980$. 
[20] G.-C. Rota. Combinatorial theory, old and new. In Proc. Internat. Cong. Math. (Nice, 1970), pages 229-233, Gauthier-Villars, Paris, 1971.

[21] W.T. Tutte. Connectivity in matroids. Canad. J. Math., 18:1301-1324, 1966.

[22] G. Whittle. Duality in polymatroids and set functions. Combin. Probab. Comput., 1:275-280, 1992.

[23] T. Zaslavsky. Signed graphs. Discrete App. Math., 4:47-74, 1982. 